Supporting Information for

\title{
Oxazaborolidinone-Mediated Asymmetric Bisvinylogous Mukaiyama Aldol Reaction
}

Alina Eggert, Christoph Etling, Lucas Millbrodt, Göran Schulz, Markus Kalesse*

Institute of Organic Chemistry, Leibniz University Hannover,

Schneiderberg 1b, 30167 Hannover

*E-Mail: markus.kalesse@oci.uni-hannover.de

\section{Table of Contents:}

General Methods

General Procedures

Analytical data of bVMAR products $\mathbf{5 a - w}$

S6-29

Determination of absolute configuration via Mosher esters

S30-32

Investigations on substrate-induced stereocontrol of aldehyde $\mathbf{4 p}$

NMR spectra

S34-97

ee-determination via chiral HPLC

S98-99

$e e$-determination via ${ }^{19} \mathrm{~F}$-spectra of Mosher esters

S100-113 


\section{General Information}

Reactions were carried out using flame-dried glass vessels under argon atmosphere. Dichloromethane was distilled under an inert atmosphere over calcium hydride. Valeronitrile was purified by distillation from $\mathrm{P}_{2} \mathrm{O}_{10}$ under nitrogen atmosphere prior to use.

$N$-Tosyl-L-tryptophan, ${ }^{1} \quad$ ethyl $\quad(2 E, 4 E)$-2-methylhexa-2,4-dienoate, ${ }^{2} \quad(E)$-3-iodo-2-methylacrylaldehyde, ${ }^{3}$ and 2-butynal dicobalt hexacarbonyl complex ${ }^{4}$ were prepared according to literature procedures.

\section{Nuclear Magnetic Resonance Spectroscopy (NMR)}

All NMR spectra were recorded at a Bruker DPX-400, AMX-400, Ascend 400 Avance II, DRX-500 or Ascend-600. ${ }^{1} \mathrm{H}$ NMR spectra were calibrated to the residual proton signal of the solvents $\left(\mathrm{CDCl}_{3}\right.$ : $\left.7.26 \mathrm{ppm}, \mathrm{C}_{6} \mathrm{D}_{6}: 7.16 \mathrm{ppm}\right) .{ }^{13} \mathrm{C}$ NMR spectra calibrated to the corresponding solvent signal $\left(\mathrm{CDCl}_{3}\right.$ : $\left.77.16 \mathrm{ppm}, \mathrm{C}_{6} \mathrm{D}_{6}: 128.06 \mathrm{ppm}\right)$.

If the samples contained a mixture of two inseparable isomers, distinguishable signals that stem from the minor isomer are marked with an asterisk*. NMR spectra were processed using TopSpin (Bruker) Version 4.0.5.

\section{Chromatography}

Thin layer chromatography was performed using silica-coated aluminum TLC-plates by MachereyNagel (layer-thickness: $0.20 \mathrm{~mm}$, pore size: $60 \mathrm{~A}$, UV-indicator $F_{254}$ ). Substanced were visualized by UV fluorescence extinction $\left(\lambda_{\max }=254 \mathrm{~nm}\right)$ and staining with basic $\mathrm{KMnO}_{4}$, acidic $p$-anisaldehyde or acidic vanillin stain.

Flash column chromatography was performed using silica by Macherey-Nagel (40-63 $\mu \mathrm{m})$.

High-performance liquid chromatography was performed using a Merck/Hitachi La Chrome®-HPLCsystem with L-7150 pump, L-7200 autosampler and L 7400-UV-detection. A Daicel Chiracel® OD-H column and an isocratic $n$-Hexane/iso-propanol (99:1) eluent was used; flow rate $1.00 \mathrm{~mL} / \mathrm{min}$.

\footnotetext{
${ }^{1}$ S. Simsek, M. Horzella, M. Kalesse, Org. Lett. 2007, 9, 26, 5637-5639; E. J. Corey, T. P. Loh, J. Am. Chem. Soc. 1991, 113, 8966-8967.

${ }^{2}$ P. Koukal, M. Kotora, Chem. Eur. J. 2015, 21, 7408-7412; E. Piers, G. L. Jung, E. H. Ruediger, Can. J. Chem. 1987, 65, 670-682.

${ }^{3}$ M. T. Gieseler, M. Kalesse, Org. Lett. 2011, 13, 2430-2432.

${ }^{4}$ S. Simsek, Dissertation, Gottfried Wilhelm Leibniz Universität Hannover, 2009.
} 


\section{High Resolution Mass Spectrometry}

Mass spectra were measured by electron-spray ionization (ESI) at a Waters Micromass LCT (TOF) with lock-spray unit und injection by loop-mode by a Waters Alliance 2695, or electron ionization (EI) at a Agilent $7890 \mathrm{GC}$ system with a 5977B detector (single-quadrupole).

\section{Optical rotation}

Optical rotations were measured on a Perkin-Elmer 341 polarimeter at the sodium D-line $\left(\lambda_{\max }=\right.$ $589.3 \mathrm{~nm}$ ), with a cell length of $\mathrm{d}=1 \mathrm{dm}$ in $\mathrm{CHCl}_{3}$. Concentrations are given in the corresponding experiment. Optical rotations were determined for reaction products with e.r. or d.r. above 5:1.

\section{Racemic samples}

Racemic samples were synthesized analogously to general procedure 2, substituting $N$-tosyl-Ltryptophan with $N$-tosylglycine.

Compounds were named as suggested by ChemDraw. 


\section{General procedure 1: Preparation of ketene silyl acetals}

To a solution of DMPU (1.20 equiv) in THF (1.9 M) was added LiHMDS (1.0 M in THF, 1.10 equiv) at $-78{ }^{\circ} \mathrm{C}$. After $30 \mathrm{~min}$, the corresponding ester $(13.0 \mathrm{mmol}, 1.00$ equiv) was added and the mixture stirred for $30 \mathrm{~min}$. After addition of the corresponding chlorosilane (14.3 mmol, 1.10 equiv), the reaction mixture was warmed to r.t. immediately and stirred for $2 \mathrm{~h}$. To the resulting orange-yellow solution, $n$ pentane was added until no more lithium salts precipitated. The mixture was transferred to a separation funnel, washed with ice-water ( $3 \times 10 \mathrm{~mL}$ ), dried over $\mathrm{Na}_{2} \mathrm{SO}_{4}$, and filtered through Celite ${ }^{\circledR}$. After evaporation of the solvent in vacuo, the so obtained KSAs were directly employed in subsequent bVMARs.

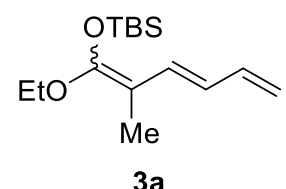

$3.44 \mathrm{~g}, 12.8 \mathrm{mmol}, 88 \%$

Prepared according to general procedure 1 starting from $2.24 \mathrm{~g}$ of $(2 E, 4 E)$-2-methylhexa-2,4-dienoate.

(E/Z 38:62, asterisk denotes minor component).

${ }^{1} \mathbf{H}$ NMR $\left(400 \mathrm{MHz}, \mathrm{CDCl}_{3}\right): \delta[\mathrm{ppm}]=6.67(\mathrm{~d}, 1 \mathrm{H}, J=15.4 \mathrm{~Hz}), 6.59 *(\mathrm{~d}, 1 \mathrm{H}, J=15.5 \mathrm{~Hz}), 6.43(\mathrm{ddt}$, $1 \mathrm{H}, J=0.9 \mathrm{~Hz}, 10.7 \mathrm{~Hz}, 16.9 \mathrm{~Hz}), 6.40 *(\mathrm{ddt}, 1 \mathrm{H}, J=0.9 \mathrm{~Hz}, 10.6 \mathrm{~Hz}, 16.9 \mathrm{~Hz}), 5.98(\mathrm{dd}, 1 \mathrm{H}, J=10.6$ $\mathrm{Hz}, 15.5 \mathrm{~Hz}), 5.13-5.06(\mathrm{~m}, 1 \mathrm{H}), 4.93-4.89(\mathrm{~m}, 1 \mathrm{H}), 3.86(\mathrm{q}, 2 \mathrm{H}, J=7.1 \mathrm{~Hz}), 3.86^{*}(\mathrm{q}, 2 \mathrm{H}, J=7.1 \mathrm{~Hz})$, $1.71 *(\mathrm{~s}, 3 \mathrm{H}), 1.67(\mathrm{~s}, 3 \mathrm{H}), 1.27(\mathrm{t}, 3 \mathrm{H}), 0.99 *(\mathrm{~s}, 9 \mathrm{H}), 0.97$ (s, 9H), $0.17(\mathrm{~s}, 6 \mathrm{H})$.

${ }^{13} \mathrm{C}$ NMR (101 MHz, $\left.\mathrm{CDCl}_{3}\right): \delta[\mathrm{ppm}]=153.0,152.7^{*}, 138.6,132.2^{*}, 132.0,124.8^{*}, 124.5,113.3^{*}$, 113.2, 98.3, 97.7*, 77.4, 66.1, 65.4*, 25.82*, 25.77, 18.4*, 18.3, 14.96*, 14.86, 11.5, 11.0*, -4.3 , $-4.4^{*}$.

HRMS due to decomposition in the mass spectrometer not available (ESI+; EI). ${ }^{5}$

Appearance: Orange oil

\footnotetext{
${ }^{5}$ This is in accordance with observations List et al. made for similar and isomeric ketene silyl acetals. See page 6 in SI: L. Ratjen, P. García-García, F. Lay, M. E. Beck, B. List, Disulfonimide-Catalyzed Asymmetric Vinylogous and Bisvinylogous Mukaiyama Aldol Reactions. Angew. Chem. 2011, 123, 780-784; Angew. Chem. Int. Ed. 2011, 50, 754-758.
} 


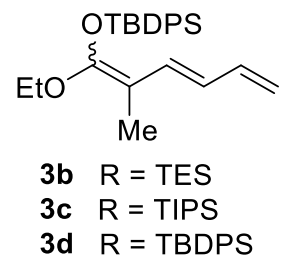

Ketene silyl acetals $\mathbf{3 b}-\mathbf{d}$ were prepared according to general procedure 1 starting from $600 \mathrm{mg}$ of (2E,4E)-2-methylhexa-2,4-dienoate. Due to their general instability these compounds were directly applied in the reactions, and used without purification and further characterization.

3b: $1.03 \mathrm{~g}, 3.85 \mathrm{mmol}, 99 \%$, orange oil.

3c: $1.11 \mathrm{~g}, 3.58 \mathrm{mmol}, 92 \%$, orange oil.

3d: $1.40 \mathrm{~g}, 3.58 \mathrm{mmol}, 92 \%$, orange oil.

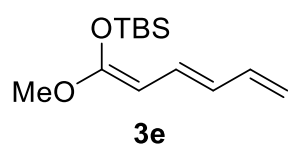

$1.26 \mathrm{~g}, 5.24 \mathrm{mmol}, 80 \%$

Prepared according to general procedure 1 starting from $825 \mathrm{mg}$ of methyl sorbate.

${ }^{1} \mathbf{H}$ NMR (400 MHz, CDCl $): \delta[\mathrm{ppm}]=6.46-6.31(\mathrm{~m}, 2 \mathrm{H}), 5.96(\mathrm{dd}, 1 \mathrm{H}, J=10.9,14.9 \mathrm{~Hz}), 5.01-4.95$ (m, 1H), 4.85-4.80 (m, 1H), 4.47 (d, 1H, $J=10.7 \mathrm{~Hz}), 3.58(\mathrm{~s}, 3 \mathrm{H}), 0.95(\mathrm{~s}, 9 \mathrm{H}), 0.17$ (s, 6H).

The spectroscopic data match the data reported by List and co-workers. ${ }^{6}$

Appearance: Yellow oil

${ }^{6}$ L. Ratjen, P. García-García, F. Lay, M. E. Beck, B. List, Angew. Chem. 2011, 123, 780-784; Angew. Chem. Int. Ed., 2011, 50, 754-758. 


\section{General procedure 2: Bisvinylogous Mukaiyama Aldol Reaction (bVMAR)}

To a suspension of $N$-tosyl-L-tryptophan ( $143 \mathrm{mg}, 400 \mu \mathrm{mol}, 1.00$ equiv) in dry $\mathrm{CH}_{2} \mathrm{Cl}_{2}(2.0 \mathrm{~mL}$ ), dichlorophenylborane ( $52 \mu \mathrm{L}, 400 \mu \mathrm{mol}, 1.00$ equiv) was added carefully at r.t. After stirring for $1 \mathrm{~h}$, the solvent was removed under reduced pressure. ${ }^{7}$ The resulting solid $\mathrm{OXB}^{8}$ was dissolved in valeronitrile $(2.0 \mathrm{~mL})$ and cooled to $-78{ }^{\circ} \mathrm{C}$. The aldehyde ( $400 \mu \mathrm{mol}, 1.00$ equiv) was added, followed by slow addition of the KSA ( $760 \mu \mathrm{mol}, 1.90$ equiv). After $2 \mathrm{~h}$, the reaction was quenched by addition of a mixture of $\mathrm{THF} / \mathrm{H}_{2} \mathrm{O} / 2 \mathrm{M} \mathrm{HCl}(5: 1: 0.2,10 \mathrm{~mL})$ and stirred vigorously for $1 \mathrm{~h}$ at r.t. To the biphasic mixture was added sat. aq. $\mathrm{NaHCO}_{3}$ solution $(5 \mathrm{~mL})$ and EtOAc $(20 \mathrm{~mL})$. After phase-separation, the aqueous phase was extracted with EtOAc $(3 \times 10 \mathrm{~mL})$. The combined organic phases were washed with brine (1x $25 \mathrm{ml}$ ), dried over $\mathrm{MgSO}_{4}$, filtered and the solvent was removed under reduced pressure. After chromatographic purification the desired ester was obtained.

\footnotetext{
${ }^{7}$ Solvent was removed at r.t. under high vacuum using a standard Schlenk line with cold trap.

${ }^{8}$ Formation of the OXB could be tracked by ${ }^{11} \mathrm{~B}-\mathrm{NMR}:\left(128 \mathrm{MHz}, \mathrm{CD}_{2} \mathrm{Cl}_{2}\right) \delta[\mathrm{ppm}]=35.9$ (broad s). However, this is not necessary since the formation is reliable and fast.
} 
Ethyl $(R, 2 E, 4 E)$-7-hydroxy-2-methyl-7-phenylhepta-2,4-dienoate (5a)<smiles>CCOC(=O)C(C)=CC=CCC(O)c1ccccc1</smiles>

$64 \mathrm{mg}, 244 \mu \mathrm{mol}, 61 \%$, e.r. $82: 18$, starting from $42.4 \mathrm{mg}$ of benzaldehyde

${ }^{1} \mathbf{H}$ NMR $\left(400 \mathrm{MHz}, \mathrm{CDCl}_{3}\right): \delta[\mathrm{ppm}]=7.38-7.34(\mathrm{~m}, 4 \mathrm{H}), 7.33-7.27(\mathrm{~m}, 1 \mathrm{H}), 7.15(\mathrm{~d}, 1 \mathrm{H}, J=$ $11.4 \mathrm{~Hz}), 6.44(\mathrm{dd}, 1 \mathrm{H}, J=11.4 \mathrm{~Hz}, 15.1 \mathrm{~Hz}), 6.04(\mathrm{dt}, 1 \mathrm{H}, J=7.4 \mathrm{~Hz}, 15.1 \mathrm{~Hz}), 4.80(\mathrm{t}, 1 \mathrm{H}, J=6.7$ $\mathrm{Hz}), 4.20(\mathrm{q}, 2 \mathrm{H}, J=7.2 \mathrm{~Hz}), 2.66(\mathrm{t}, 2 \mathrm{H}, J=6.7 \mathrm{~Hz}), 2.00(\mathrm{br} \mathrm{s}, 1 \mathrm{H}), 1.92(\mathrm{~s}, 3 \mathrm{H}), 1.30(\mathrm{t}, 3 \mathrm{H}, J=7.2$ $\mathrm{Hz})$.

${ }^{13} \mathrm{C} \mathrm{NMR}\left(101 \mathrm{MHz}, \mathrm{CDCl}_{3}\right): \delta[\mathrm{ppm}]=168.7,143.8,137.9,137.6,129.2,128.7,127.9,126.6,125.9$, 73.8, 60.7, 43.2, 14.5, 12.8 .

HRMS (ESI-TOF) m/z: [M+H] $]^{+}$Calcd for $\mathrm{C}_{16} \mathrm{H}_{21} \mathrm{O}_{3}$ 261.1491; Found 261.1487.

Column chromatography: PE:EtOAc 5:1

$\mathbf{R}_{f}=0.40$ (PE:EtOAc 5:1)

$[\alpha]_{\mathbf{D}}{ }^{20}=+13.6\left(\mathrm{c}=8.8 \mathrm{mg} / \mathrm{mL}, \mathrm{CHCl}_{3}\right)$

Appearance: Yellow oil 
Ethyl $(R, 2 E, 4 E)$-7-hydroxy-2-methyl-7-(o-tolyl)hepta-2,4-dienoate (5b)

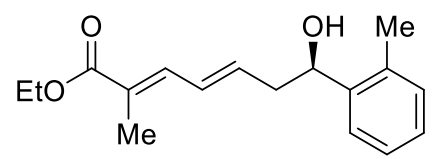

$65.9 \mathrm{mg}, 240 \mu \mathrm{mol}, 46 \%$, e.r. $65: 35$, starting from $48.0 \mathrm{mg}$ of 2-methyl benzaldehyde

${ }^{1} \mathbf{H}$ NMR $\left(400 \mathrm{MHz}, \mathrm{CDCl}_{3}\right): \delta[\mathrm{ppm}]=7.49(\mathrm{~d}, 1 \mathrm{H}, J=7.4 \mathrm{~Hz}), 7.24-7.12(\mathrm{~m}, 4 \mathrm{H}), 6.45(\mathrm{dd}, 1 \mathrm{H}, J=$ $11.1 \mathrm{~Hz}, 15.1 \mathrm{~Hz}), 6.10(\mathrm{dt}, 1 \mathrm{H}, J=7.5 \mathrm{~Hz}, 15.1 \mathrm{~Hz}), 5.03(\mathrm{dd}, 1 \mathrm{H}, J=5.5 \mathrm{~Hz}, 7.0 \mathrm{~Hz}), 4.20(\mathrm{q}, 2 \mathrm{H}, J$ $=7.1 \mathrm{~Hz}), 2.64-2.58(\mathrm{~m}, 2 \mathrm{H}), 2.37(\mathrm{~s}, 3 \mathrm{H}), 1.93-1.92(\mathrm{~m}, 3 \mathrm{H}), 1.30(\mathrm{t}, 3 \mathrm{H}, J=7.1 \mathrm{~Hz})$.

${ }^{13} \mathbf{C}$ NMR (101 MHz, $\left.\mathrm{CDCl}_{3}\right): \delta[\mathrm{ppm}]=168.7,141.9,137.9,134.6,130.7,127.8,126.6,125.3,70.1$, $60.7,42.0,19.2,14.5,12.8$.

HRMS (ESI-TOF) m/z: [M+Na] $]^{+}$Calcd for $\mathrm{C}_{17} \mathrm{H}_{22} \mathrm{O}_{3} \mathrm{Na}$ 297.1467; Found 297.1468.

Column chromatography: PE:EtOAc 5:1

$\mathbf{R}_{f}=0.33$ (PE:EtOAc 5:1)

$[\boldsymbol{\alpha}]_{\mathbf{D}}{ }^{20}=+17.8\left(\mathrm{c}=10.0 \mathrm{mg} / \mathrm{mL}, \mathrm{CHCl}_{3}\right)$

Appearance: Yellow oil 


\section{Ethyl $(R, 2 E, 4 E)-7-h y d r o x y-7-(4-m e t h o x y p h e n y l)-2-m e t h y l h e p t a-2,4-d i e n o a t e ~(5 c)$}<smiles>CCOC(=O)C(C)=CC=CCC(O)c1ccc(OC)cc1</smiles>

$44.1 \mathrm{mg}, 152 \mu \mathrm{mol}, 38 \%$, e.r. $78: 22$, starting from $54.4 \mathrm{mg}$ of $p$-anisaldehyde, obtained as 3.6:1 mixture of the $2 E$ and $2 Z$ isomer. $^{9}$

${ }^{1} \mathbf{H}$ NMR $\left(400 \mathrm{MHz}, \mathrm{C}_{6} \mathrm{D}_{6}\right): \delta[\mathrm{ppm}]=7.79-7.72(2 \mathrm{Z}, \mathrm{m}, 1 \mathrm{H}), 7.42-7.36(2 E, \mathrm{~m}, 1 \mathrm{H}), 7.14-7.09(\mathrm{~m}$, 2H), 6.81-6.74 (m, 2H), 6.31-6.22 (m, 1H), 5.90-5.80 (2E, m, 1H), 5.78-5.70 (2Z, m, 1H), 4.47-4.38 $(\mathrm{m}, 1 \mathrm{H}), 4.04(2 \mathrm{Z}, \mathrm{q}, 2 \mathrm{H}, J=7.1 \mathrm{~Hz}), 4.03(2 E, \mathrm{q}, 2 \mathrm{H}, J=7.1 \mathrm{~Hz}), 3.31(\mathrm{~s}, 3 \mathrm{H}), 2.71-2.62(2 Z, \mathrm{~m}, 1 \mathrm{H})$, 2.61-2.52 (2Z, m, 1H), 2.51-2.42 (2E, m, 1H), 2.41-2.32 (2E, m, 1H), 1.91-1.88 (m, 3H), 1.61 (br s, $1 \mathrm{H}), 0.99(2 E, \mathrm{t}, 3 \mathrm{H}, J=7.1 \mathrm{~Hz}), 0.98(2 Z, \mathrm{t}, 3 \mathrm{H}, J=7.2 \mathrm{~Hz})$.

${ }^{13}$ C NMR (101 MHz, $\left.\mathrm{C}_{6} \mathrm{D}_{6}\right): \delta[\mathrm{ppm}]=168.2(2 Z), 168.1(2 E), 159.62(2 E), 159.60(2 Z), 138.4(2 E)$, 138.3 (2E), 136.8 (2E), 136.7 (2Z), 135.3 (2Z), 132.9 (2E), 128.9 (2E), 127.4 (2Z), 127.3 (2E), 126.6 (2E), 126.1 (2Z), 114.08 (2E), 114.06 (2Z), 73.4 (2Z), 73.3 (2E), 60.6 (2Z), 60.5 (2E), $54.8(2 E), 54.8$ (2Z), $43.6(2 E), 38.2(2 Z), 14.36(2 E), 14.35(2 Z), 12.8(2 E), 12.7(2 Z)$.

HRMS (ESI-TOF) m/z: [M+Na $]^{+}$Calcd for $\mathrm{C}_{17} \mathrm{H}_{22} \mathrm{O}_{4} \mathrm{Na}$ 313.1416; Found 313.1404.

\section{Column chromatography: PE:EtOAc 5:1}

$\mathbf{R}_{f}=0.13$ (PE:EtOAc 5:1)

$[\boldsymbol{\alpha}]_{\mathrm{D}}{ }^{20}=+8.3\left(\mathrm{c}=10.0 \mathrm{mg} / \mathrm{mL}, \mathrm{CHCl}_{3}\right)$

Appearance: Yellow oil

\footnotetext{
${ }^{9}$ Assignment of $E$ and $Z$ isomer based on the relative magnitudes of the ${ }^{4} J_{\mathrm{H}, \mathrm{H}}$ between the $\mathrm{C} 2$ Me group and the olefinic proton at C3. See M. Barfield, R. J. Spear, S. Sternhell, Chem. Rev. 1976, 76, 593-624.
} 
Ethyl $(R, 2 E, 4 E)-7-h y d r o x y-2-m e t h y l-7-(4-n i t r o p h e n y l) h e p t a-2,4-d i e n o a t e ~(5 d)$<smiles>CCOC(=O)C(C)=CC=CC[C@H](O)c1ccc([N+](=O)[O-])cc1</smiles>

$56.1 \mathrm{mg}, 184 \mu \mathrm{mol}, 71 \%$, e.r. $86: 14$, starting from $60.4 \mathrm{mg}$ of $p$-nitro benzaldehyde.

${ }^{1} \mathbf{H}$ NMR $\left(400 \mathrm{MHz}, \mathrm{CDCl}_{3}\right): \delta[\mathrm{ppm}]=8.26-8.16(\mathrm{~m}, 2 \mathrm{H}), 7.57-7.50(\mathrm{~m}, 2 \mathrm{H}), 7.13(\mathrm{~d}, 1 \mathrm{H}, J=11.2 \mathrm{~Hz})$, 6.49-6.36 (m, 1H), 6.00 (dt, 1H, J = 7.5 Hz, 15.1 Hz), 4.96-4.87 (m, 1H), 4.25-4.15 (m, 2H), 2.70-2.59 $(\mathrm{m}, 2 \mathrm{H}), 1.90(\mathrm{~s}, 3 \mathrm{H}), 1.32-1.27(\mathrm{~m}, 3 \mathrm{H})$.

${ }^{13} \mathbf{C}$ NMR $\left(101 \mathrm{MHz}, \mathrm{CDCl}_{3}\right): \delta[\mathrm{ppm}]=168.6,151.2,147.4,137.5,136.2,129.8,127.1,126.7,123.8$, $72.5,60.8,43.2,14.4,12.7$.

HRMS (ESI-TOF) m/z: [M+Na $]^{+}$Calcd for $\mathrm{C}_{16} \mathrm{H}_{19} \mathrm{NO}_{5} \mathrm{Na}$ 328.1161; Found 328.1163.

Column chromatography: PE:EtOAc 4:1 to $1: 1$

$\mathbf{R}_{f}=0.08($ PE:EtOAc 5:1)

$[\boldsymbol{\alpha}]_{\mathbf{D}^{20}}=+22.4\left(\mathrm{c}=10.0 \mathrm{mg} / \mathrm{mL}, \mathrm{CHCl}_{3}\right)$

Appearance: Orange oil 


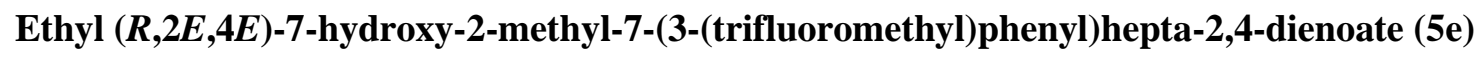<smiles>CCOC(=O)C(C)=CC=CCC(O)c1cccc(C(F)(F)F)c1</smiles>

$85.3 \mathrm{mg}, 260 \mu \mathrm{mol}, 55 \%$, e.r. $81: 19$, starting from $69.6 \mathrm{mg}$ of 3-trifluoromethyl benzaldehyde.

${ }^{1}$ H NMR (400 MHz, CDCl $): \delta[p p m]=7.65(\mathrm{~s}, 1 \mathrm{H}), 7.58-7.53(\mathrm{~m}, 2 \mathrm{H}), 7.50-7.45(\mathrm{~m}, 1 \mathrm{H}), 7.17-7.12$ $(\mathrm{m}, 1 \mathrm{H}), 6.49-6.40(\mathrm{~m}, 1 \mathrm{H}), 6.07-5.99(\mathrm{~m}, 1 \mathrm{H}), 4.86(\mathrm{dd}, 1 \mathrm{H}, J=5.4 \mathrm{~Hz}, 5.4 \mathrm{~Hz}), 4.20(\mathrm{q}, 2 \mathrm{H}, J=7.1$ $\mathrm{Hz}), 2.68-2.60(\mathrm{~m}, 2 \mathrm{H}), 1.93-1.91(\mathrm{~m}, 3 \mathrm{H}), 1.30(\mathrm{t}, 3 \mathrm{H}, J=7.1 \mathrm{~Hz})$.

${ }^{13} \mathbf{C}$ NMR (101 MHz, $\left.\mathrm{CDCl}_{3}\right): \delta[\mathrm{ppm}]=168.6,144.7,137.5,136.5,130.9\left(\mathrm{q}, J_{\mathrm{C}, \mathrm{F}}=32.3 \mathrm{~Hz}\right), 129.6$, 129.2, 129.0, 126.9, $124.6\left(\mathrm{q}, J_{\mathrm{C}, \mathrm{F}}=3.9 \mathrm{~Hz}\right), 124.1\left(\mathrm{q}, J_{\mathrm{C}, \mathrm{F}}=272.0 \mathrm{~Hz}\right), 122.6\left(\mathrm{q}, J_{\mathrm{C}, \mathrm{F}}=3.9 \mathrm{~Hz}\right), 73.0$, $60.8,43.3,14.4,12.8$.

HRMS (ESI-TOF) m/z: [M+Na] ${ }^{+}$Calcd for $\mathrm{C}_{17} \mathrm{H}_{19} \mathrm{O}_{3} \mathrm{NaF}_{3}$ 351.1184; Found 351.1184.

Column chromatography: PE:EtOAc 4:1 to 2:1

$\mathbf{R}_{f}=0.25(\mathrm{PE}:$ EtOAc 5:1)

$[\boldsymbol{\alpha}]_{\mathbf{D}}{ }^{20}=+20.6\left(\mathrm{c}=10.0 \mathrm{mg} / \mathrm{mL}, \mathrm{CHCl}_{3}\right)$

Appearance: Yellow oil 


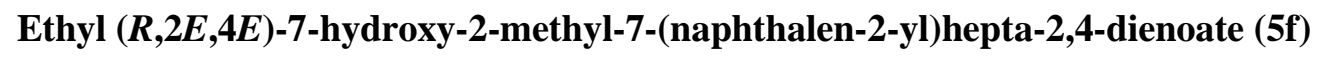<smiles>CCOC(=O)C(=CC=CCC(O)c1ccc2ccccc2c1)[N+](=O)[O-]</smiles>

$51 \mathrm{mg}, 184 \mu \mathrm{mol}, 40 \%$, e.r. 80:20, starting from $62.5 \mathrm{mg}$ of 2-naphthaldehyde.

${ }^{1} \mathbf{H}$ NMR $\left(400 \mathrm{MHz}, \mathrm{C}_{6} \mathrm{D}_{6}\right): \delta[\mathrm{ppm}]=7.70-7.59(\mathrm{~m}, 4 \mathrm{H}), 7.43-7.37(\mathrm{~m}, 1 \mathrm{H}), 7.34-7.24(\mathrm{~m}, 3 \mathrm{H})$, 6.32-6.23 (m, 1H), 5.89-5.80 (m, 1H), 4.55-4.48 (m, 1H), $4.05(\mathrm{q}, 2 \mathrm{H}, J=7.1 \mathrm{~Hz}), 2.53-2.36(\mathrm{~m}, 2 \mathrm{H})$, 1.91-1.89 (m, 3H), 1.44 (br s, 1H), 0.99 (t, 3H, $J=7.1 \mathrm{~Hz}$ ).

${ }^{13} \mathbf{C ~ N M R}\left(101 \mathrm{MHz}, \mathrm{CDCl}_{3}\right): \delta[\mathrm{ppm}]=168.6,141.1,137.9,137.5,133.4,133.2,129.2,128.5,128.1$, $127.8,126.6,126.4,126.1,124.7,124.0,73.9,60.7,43.1,14.4,12.7$.

HRMS (ESI-TOF) m/z: [M+Na] $]^{+}$Calcd for $\mathrm{C}_{20} \mathrm{H}_{22} \mathrm{O}_{3} \mathrm{Na}$ 333.1467; Found 333.1468.

Column chromatography: PE:EtOAc 5:1

$\mathbf{R}_{f}=0.32$ (PE:EtOAc 5:1)

$[\alpha]_{\mathrm{D}}{ }^{20}=+63.2\left(\mathrm{c}=10.0 \mathrm{mg} / \mathrm{mL}, \mathrm{CHCl}_{3}\right)$

Appearance: Waxy solid 


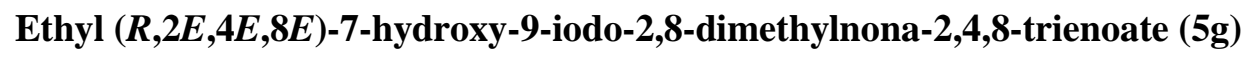<smiles>CCOC(=O)C(C=CC=CCC(O)C(=CI)[N+](=O)[O-])=COC</smiles>

$91 \mathrm{mg}, 260 \mu \mathrm{mol}, 65 \%$, e.r. 89:11, starting from $78.4 \mathrm{mg}$ of 3-iodomethacrolein.

${ }^{1} \mathbf{H}$ NMR $\left(400 \mathrm{MHz}, \mathrm{CDCl}_{3}\right): \delta[\mathrm{ppm}]=7.15(\mathrm{~d}, 1 \mathrm{H}, J=11.4 \mathrm{~Hz}), 6.48-6.39(\mathrm{~m}, 1 \mathrm{H}), 6.34(\mathrm{~s}, 1 \mathrm{H}), 5.98$ $(\mathrm{dt}, 1 \mathrm{H}, J=7.4,14.9 \mathrm{~Hz}), 4.30-4.25(\mathrm{~m}, 1 \mathrm{H}), 4.21(\mathrm{q}, 2 \mathrm{H}, J=7.1 \mathrm{~Hz}), 2.52-2.38(\mathrm{~m}, 2 \mathrm{H}), 1.94(\mathrm{~s}, 3 \mathrm{H})$, $1.85(\mathrm{~s}, 3 \mathrm{H}), 1.30(\mathrm{t}, 3 \mathrm{H}, J=7.1 \mathrm{~Hz})$.

${ }^{13} \mathrm{C} \mathrm{NMR}\left(101 \mathrm{MHz}, \mathrm{CDCl}_{3}\right): \delta[\mathrm{ppm}]=168.6,149.1,137.6,136.6,129.3,126.9,79.0,75.8,60.7,39.3$, $20.3,14.5,12.8$.

HRMS (ESI-TOF) m/z: [M+Na $]^{+}$Calcd for $\mathrm{C}_{13} \mathrm{H}_{19} \mathrm{O}_{3} \mathrm{NaI}$ 373.0277; Found 373.0275.

Column chromatography: PE:EtOAc 5:1

$\mathbf{R}_{f}=0.44($ PE:EtOAc 5:1)

$[\boldsymbol{\alpha}]_{\mathbf{D}}{ }^{20}=-228.6\left(\mathrm{c}=10.0 \mathrm{mg} / \mathrm{mL}, \mathrm{CHCl}_{3}\right)$

Appearance: Yellow oil 


\section{Preparation of protected dicobalt aldehyde $4 \mathrm{~m}$ and oxidative liberation of bVMAR product $5 \mathrm{~h}:{ }^{4}$}

Preparation of aldehyde 4h:

To a solution of dicobalt octacarbonyl ( $818 \mathrm{mg}, 2.39 \mathrm{mmol}, 1.00$ equiv) in $\mathrm{CH}_{2} \mathrm{Cl}_{2}$ (48 $\mathrm{ml} 0.05 \mathrm{M}$ ) was slowly added 2-butyne diethyl acetal ( $380 \mu \mathrm{l}, 2.39 \mathrm{mmol}, 1.00$ equiv) at rt. After stirring over night at this temperature, the reaction mixture was passed through a short plug of basic aluminium oxide and the filtrate was concentrated under reduced pressure. The residue was then dissolved in acetone $(8 \mathrm{ml}, 0.3$ M) and water $(170 \mu \mathrm{l})$ and amberlyst $15(272 \mathrm{mg})$ were added. After stirring for $3 \mathrm{~h}$ at rt, the solution was filtered through Celite ${ }^{\circledR}$ and the filtrate was concentrated under reduced pressure. Purification by column chromatography (PE:MTBE 40:1) yielded aldehyde 4h (651 mg, $1.84 \mathrm{mmol}, 77 \%$ ) as a red oil, which was used without further purification.

Oxidative liberation of bVMAR product 5h:

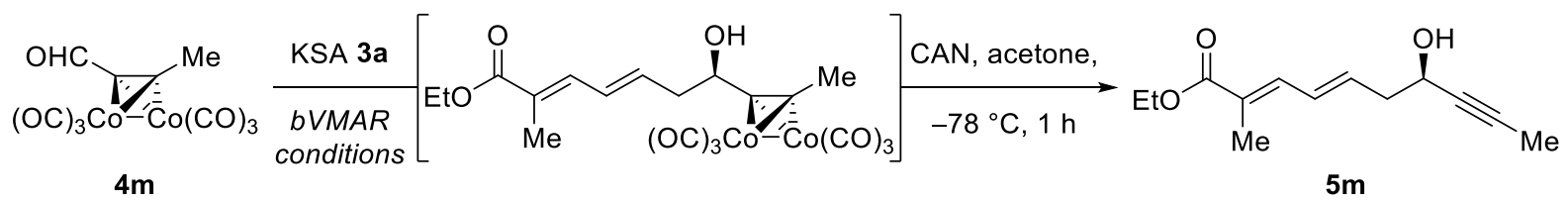

After following General Procedure 2 for the bVMAR of KSA 3a with aldehyde $\mathbf{4 h}$, the crude product was passed through a short silica column and the concentrated filtrate was dissolved in acetone $(30 \mathrm{ml})$. To this solution was added ceric ammonium nitrate $(1.10 \mathrm{~g}, 2.00 \mathrm{mmol}, 5.00$ equiv) in acetone ( $5 \mathrm{ml})$ at $-78{ }^{\circ} \mathrm{C}$. After $1 \mathrm{~h}$, brine was added and the mixture was warmed to rt. The phases were separated and the aqueous layer was extracted with MTBE $(3 \times 30 \mathrm{ml})$. The combined organic layers were washed with sat. aq. $\mathrm{NaHCO}_{3}$ solution (1x $75 \mathrm{ml}$ ) and brine (1x $75 \mathrm{ml}$ ), dried over $\mathrm{MgSO}_{4}$. Purification by flash column chromatography yielded the desired bVMAR product $\mathbf{5 h}$.

Ethyl $(R, 2 E, 4 E)$-7-hydroxy-2-methyldeca-2,4-dien-8-ynoate (5h)<smiles>CC#CC(O)C/C=C/C=C(/C(=O)OCC)[N+](=O)[O-]</smiles>

$49 \mathrm{mg}, 220 \mu \mathrm{mol}, 55 \%$, e.r. 90:10, starting from $141.6 \mathrm{mg}$ of aldehyde $\mathbf{4 h}$.

${ }^{1} \mathbf{H}$ NMR $\left(400 \mathrm{MHz}, \mathrm{CDCl}_{3}\right): \delta[\mathrm{ppm}]=7.21-7.15(\mathrm{~m}, 1 \mathrm{H}), 6.50-6.44(\mathrm{~m}, 1 \mathrm{H}), 6.16-6.09(\mathrm{~m}, 1 \mathrm{H})$, $4.49-4.40$ (m, 1H), 4.20 (q, 2H, $J=7.1 \mathrm{~Hz}), 2.56(\mathrm{t}, 2 \mathrm{H}, J=6.5 \mathrm{~Hz}), 1.94(\mathrm{~s}, 3 \mathrm{H}), 1.85$ (d, 3H, $J=2.1$ $\mathrm{Hz}), 1.30(\mathrm{t}, 3 \mathrm{H}, 7.1 \mathrm{~Hz})$. 
${ }^{13} \mathbf{C}$ NMR $\left(101 \mathrm{MHz}, \mathrm{CDCl}_{3}\right): \delta[\mathrm{ppm}]=168.7,137.9,136.5,129.5,126.7,82.1,79.6,62.0,60.7,41.9$, $14.4,12.8,3.7$.

HRMS (ESI-TOF) m/z: [M+Na] ${ }^{+}$Calcd for $\mathrm{C}_{13} \mathrm{H}_{18} \mathrm{O}_{3} \mathrm{Na}$ 245.1154; Found 245.1148.

Column chromatography: PE:EtOAc 10:1 to 5:1

$\mathbf{R}_{f}=0.43$ (PE:EtOAc 4:1)

$[\alpha]_{\mathbf{D}}^{20}=+19.5\left(\mathrm{c}=8.2 \mathrm{mg} / \mathrm{mL}, \mathrm{CHCl}_{3}\right)$

Appearance: Colorless oil 
Ethyl $(S, 2 E, 4 E)-7-h y d r o x y-2-m e t h y l u n d e c a-2,4-d i e n o a t e ~(5 i)$

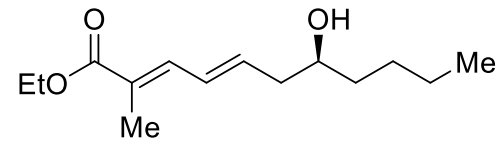

$68 \mathrm{mg}, 284 \mu \mathrm{mol}, 71 \%$, e.r. $87: 13$, starting from $34.4 \mathrm{mg}$ of valeraldehyde.

${ }^{1} \mathbf{H}$ NMR $\left(400 \mathrm{MHz}, \mathrm{CDCl}_{3}\right): \delta[\mathrm{ppm}]=7.20-7.14(\mathrm{~m}, 1 \mathrm{H}), 6.48-6.38(\mathrm{~m}, 1 \mathrm{H}), 6.14-6.04(\mathrm{~m}, 1 \mathrm{H}), 4.20$ (q, 2H, $J=7.1 \mathrm{~Hz}), 3.74-3.67(\mathrm{~m}, 1 \mathrm{H}), 2.46-2.38(\mathrm{~m}, 1 \mathrm{H}), 2.35-2.25(\mathrm{~m}, 1 \mathrm{H}), 1.93(\mathrm{~m}, 3 \mathrm{H}), 1.56$ (br s, $1 \mathrm{H}), 1.53-1.27(\mathrm{~m}, 9 \mathrm{H}), 0.94-0.89(\mathrm{~m}, 3 \mathrm{H})$.

${ }^{13} \mathrm{C}$ NMR $\left(101 \mathrm{MHz}, \mathrm{CDCl}_{3}\right): \delta[\mathrm{ppm}]=168.7,138.3,138.0,129.0,126.3,71.2,60.7,41.5,36.9,28.0$, $22.8,14.5,14.2,12.8$

HRMS (ESI-TOF) m/z: [M+Na] $]^{+}$Calcd for $\mathrm{C}_{14} \mathrm{H}_{24} \mathrm{O}_{3} \mathrm{Na}$ 263.1623; Found 263.1617.

Column chromatography: PE:EtOAc 10:1

$\mathbf{R}_{f}=0.45(\mathrm{PE}: \mathrm{EtOAc} 4: 1)$

$[\boldsymbol{\alpha}]_{\mathbf{D}} \mathbf{2 0}^{\mathbf{2 0}}=+7.8\left(\mathrm{c}=20.6 \mathrm{mg} / \mathrm{mL}, \mathrm{CHCl}_{3}\right)$

Appearance: Yellow oil 
Ethyl (S,2E,4E)-7-hydroxy-15-((4-methoxybenzyl)oxy)-2-methylpentadeca-2,4-dienoate (5j)

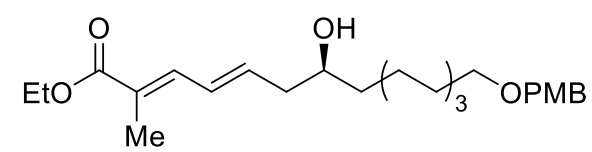

$87 \mathrm{mg}, 200 \mu \mathrm{mol}, 50 \%$ (75\% brsm), e.r. 86:14, starting from $111.4 \mathrm{mg}$ of aldehyde $\mathbf{4 j}$.

${ }^{1} \mathbf{H}$ NMR $\left(400 \mathrm{MHz}, \mathrm{C}_{6} \mathrm{D}_{6}\right): \delta[\mathrm{ppm}]=7.49-7.43(\mathrm{~m}, 1 \mathrm{H}), 7.29-7.24(\mathrm{~m}, 2 \mathrm{H}), 6.85-6.80(\mathrm{~m}, 2 \mathrm{H}), 6.36-$ $6.26(\mathrm{~m}, 1 \mathrm{H}), 5.88-5.78(\mathrm{~m}, 1 \mathrm{H}), 4.38(\mathrm{~s}, 2 \mathrm{H}), 4.09(\mathrm{q}, 2 \mathrm{H}, J=7.2 \mathrm{~Hz}), 3.41-3.36(\mathrm{~m}, 3 \mathrm{H}), 3.32(\mathrm{~s}, 3 \mathrm{H})$, 2.13-2.00 (m, 2H), 1.97-1.95 (m, 3H), 1.69-1.60 (m, 2H), 1.48-1.18 (m, 12H), 0.89 (br s, 1H), 1.02 (t, $3 \mathrm{H}, J=7.1 \mathrm{~Hz})$.

${ }^{13} \mathrm{C}$ NMR $\left(101 \mathrm{MHz}, \mathrm{C}_{6} \mathrm{D}_{6}\right): \delta[\mathrm{ppm}]=168.1,159.7,138.9,138.4,131.6,129.4,128.7,126.4,114.1$, $72.8,70.9,70.3,60.5,54.8,41.8,37.5,30.4,30.0,30.0,29.9,26.8,26.0,14.4,12.9$.

HRMS (ESI-TOF) m/z: [M+Na] $]^{+}$Calcd for $\mathrm{C}_{26} \mathrm{H}_{40} \mathrm{O}_{5} \mathrm{Na}$ 455.2773; Found 455.2773.

Column chromatography: PE:EtOAc 5:1

$\mathbf{R}_{f}=0.20($ PE:EtOAc 4:1)

$[\boldsymbol{\alpha}]_{\mathbf{D}^{20}}=+4.3\left(\mathrm{c}=7.0 \mathrm{mg} / \mathrm{mL}, \mathrm{CHCl}_{3}\right)$

Appearance: Amorphous solid 


\section{Ethyl $(R, 2 E, 4 E)-7-h y d r o x y-2,8-d i m e t h y l n o n a-2,4-d i e n o a t e ~(5 k)$}<smiles>CCOC(=O)C(=CC=CCC(O)C(C)C)[N+](=O)[O-]</smiles>

$59 \mathrm{mg}, 261 \mu \mathrm{mol}, 65 \%$, e.r. $95: 5$, starting from $28.8 \mathrm{mg}$ of isobutyraldehyde.

$1.2 \mathrm{mmol}$ scale: $92.7 \mathrm{mg}, 0.41 \mathrm{mmol}, 34 \%$, e.r. $92: 8$, starting from $86.4 \mathrm{mg}$ of isobutyraldehyde.

${ }^{1}$ H NMR (400 MHz, CDCl $): \delta[p p m]=7.20-7.15(\mathrm{~m}, 1 \mathrm{H}), 6.49-6.40(\mathrm{~m}, 1 \mathrm{H}), 6.15-6.06(\mathrm{~m}, 1 \mathrm{H}), 4.20$ (q, $2 \mathrm{H}, J=7.1 \mathrm{~Hz}), 3.50-3.43(\mathrm{~m}, 1 \mathrm{H}), 2.47-2.38(\mathrm{~m}, 1 \mathrm{H}), 2.34-2.24(\mathrm{~m}, 1 \mathrm{H}), 1.95-1.92(\mathrm{~m}, 3 \mathrm{H}), 1.75-$ $1.66(\mathrm{~m}, 1 \mathrm{H}), 1.55(\mathrm{br} \mathrm{s}, 1 \mathrm{H}), 1.30(\mathrm{t}, 3 \mathrm{H}, J=7.1 \mathrm{~Hz}), 0.96(\mathrm{~d}, 3 \mathrm{H}, J=2.5 \mathrm{~Hz}), 0.94(\mathrm{~d}, 3 \mathrm{H}, J=2.5 \mathrm{~Hz})$.

${ }^{13} \mathbf{C}$ NMR $\left(101 \mathrm{MHz}, \mathrm{CDCl}_{3}\right): \delta[\mathrm{ppm}]=168.7,138.9,138.0,128.8,126.2,75.9,60.7,38.4,33.5,18.9$, $17.5,14.5,12.8$.

HRMS (ESI-TOF) m/z: [M+Na] ${ }^{+}$Calcd for $\mathrm{C}_{13} \mathrm{H}_{22} \mathrm{O}_{3} \mathrm{Na}$ 249.1467; Found 249.1477.

Column chromatography: PE:EtOAc 10:1

$\mathbf{R}_{f}=0.42(\mathrm{PE}: \mathrm{EtOAc} 4: 1)$

$[\alpha]_{\mathrm{D}}{ }^{20}=+12.6\left(\mathrm{c}=9.5 \mathrm{mg} / \mathrm{mL}, \mathrm{CHCl}_{3}\right)$

Appearance: Pale-yellow oil

bVMAR with isobutyraldehyde at $1.2 \mathrm{mmol}$ scale:

To a suspension of $N$-tosyl-L-tryptophan (430 mg, $1.20 \mathrm{mmol}, 1.00$ equiv) in dry $\mathrm{CH}_{2} \mathrm{Cl}_{2}(6.0 \mathrm{~mL})$, dichlorophenylborane ( $156 \mu \mathrm{L}, 1.20 \mathrm{mmol}, 1.00$ equiv) was added carefully at r.t. After stirring for $1 \mathrm{~h}$, the solvent was removed under reduced pressure. The resulting solid OXB was dissolved in valeronitrile $(6.0 \mathrm{~mL})$ and cooled to $-78{ }^{\circ} \mathrm{C}$. Isobutyraldehyde ( $87 \mathrm{mg}, 1.20 \mathrm{mmol}, 1.00$ equiv) was added, followed by slow addition of the KSA 3a ( $611 \mathrm{mg}, 2.28 \mathrm{mmol}, 1.90$ equiv). After $2 \mathrm{~h}$, the reaction was quenched by addition of a mixture of $\mathrm{THF} / \mathrm{H}_{2} \mathrm{O} / 2 \mathrm{M} \mathrm{HCl}(5: 1: 0.2,30 \mathrm{~mL})$ and stirred vigorously for $1 \mathrm{~h}$ at r.t. To the biphasic mixture was added sat. aq. $\mathrm{NaHCO}_{3}$-solution $(15 \mathrm{~mL})$ and EtOAc $(60 \mathrm{~mL})$. After phaseseparation, the aqueous phase was extracted with EtOAc $(3 \times 30 \mathrm{~mL})$. The combined organic phases were washed with brine (1x $100 \mathrm{ml})$, dried over $\mathrm{MgSO}_{4}$, filtered and the solvent was removed under reduced pressure. After chromatographic purification bVMAR product 5k $(92.1 \mathrm{mg}, 0.41 \mathrm{mmol}, 34 \%$, e.r. 92:8) was obtained as a pale-yellow oil. 


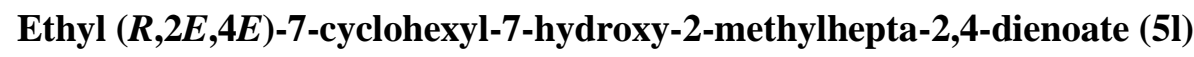

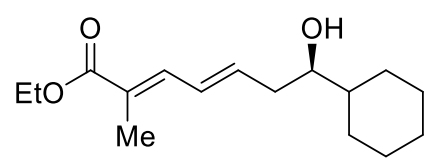

$47.5 \mathrm{mg}, 172 \mu \mathrm{mol}, 45 \%$, e.r. $90: 10$, starting from $44.9 \mathrm{mg}$ of cyclohexyl carbaldehyde.

${ }^{1} \mathbf{H}$ NMR (400 MHz, CDCl $): \delta[\mathrm{ppm}]=7.21-7.15(\mathrm{~m}, 1 \mathrm{H}), 6.48-6.39(\mathrm{~m}, 1 \mathrm{H}), 6.15-6.06(\mathrm{~m}, 1 \mathrm{H})$, 4.20 (q, 2H, $J=7.2 \mathrm{~Hz}), 3.49-3.43(\mathrm{~m}, 1 \mathrm{H}), 2.48-2.39$ (m, 1H), 2.35-2.24 (m, 1H), 1.94-1.92 (m, 3H), $1.88-1.63(\mathrm{~m}, 6 \mathrm{H}), 1.42-0.9(\mathrm{~m}, 5 \mathrm{H}), 1.29(\mathrm{t}, 3 \mathrm{H}, J=7.2 \mathrm{~Hz})$.

${ }^{13} \mathrm{C} \mathrm{NMR}\left(101 \mathrm{MHz}, \mathrm{CDCl}_{3}\right): \delta[\mathrm{ppm}]=168.7,139.1,138.1,128.8,126.1,75.3,60.7,43.4,38.4,29.3$, $28.1,26.6,26.4,26.2,14.4,12.8$.

HRMS (ESI-TOF) m/z: [M+Na] ${ }^{+}$Calcd for $\mathrm{C}_{16} \mathrm{H}_{26} \mathrm{O}_{3} \mathrm{Na} 289.1780$; Found 289.1788.

Column chromatography: PE:EtOAc 5:1

$\mathbf{R}_{f}=0.36($ PE:EtOAc 5:1)

$[\alpha]_{\mathrm{D}}{ }^{20}=+6.2\left(\mathrm{c}=9.2 \mathrm{mg} / \mathrm{mL}, \mathrm{CHCl}_{3}\right)$

Appearance: Yellow oil 
Ethyl $(R, 2 E, 4 E)$-7-hydroxy-2,8,8-trimethylnona-2,4-dienoate (5m)

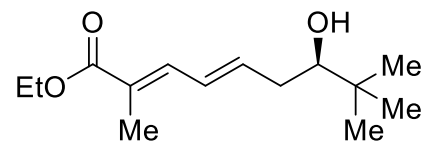

$62 \mathrm{mg}, 258 \mu \mathrm{mol}, 65 \%$, e.r. $95: 5$, starting from $34.4 \mathrm{mg}$ of pivaldehyde.

${ }^{1} \mathbf{H}$ NMR $\left(400 \mathrm{MHz}, \mathrm{CDCl}_{3}\right): \delta[\mathrm{ppm}]=7.21-7.15(\mathrm{~m}, 1 \mathrm{H}), 6.49-6.40(\mathrm{~m}, 1 \mathrm{H}), 6.18-6.09(\mathrm{~m}, 1 \mathrm{H}), 4.20$ (q, 2H, $J=7.1 \mathrm{~Hz}), 3.33-3.31(\mathrm{~m}, 1 \mathrm{H}), 2.52-2.41(\mathrm{~m}, 1 \mathrm{H}), 2.22-2.11(\mathrm{~m}, 1 \mathrm{H}), 1.93(\mathrm{~s}, 3 \mathrm{H}), 1.53$ (br s, $1 \mathrm{H}), 1.30(\mathrm{t}, 3 \mathrm{H}, J=7.2 \mathrm{~Hz}), 0.93(\mathrm{~s}, 9 \mathrm{H})$.

${ }^{13} \mathrm{C} \mathrm{NMR}\left(101 \mathrm{MHz}, \mathrm{CDCl}_{3}\right): \delta[\mathrm{ppm}]=168.7,140.2,138.1,128.6,126.1,78.9,60.7,36.1,35.1,25.8$, $14.5,12.8$.

HRMS (ESI-TOF) m/z: [M+Na] ${ }^{+}$Calcd for $\mathrm{C}_{14} \mathrm{H}_{24} \mathrm{O}_{3} \mathrm{Na}$ 263.1623; Found 263.1630.

Column chromatography: PE:EtOAc 10:1

$\mathbf{R}_{f}=0.55$ (PE:EtOAc 4:1)

$[\boldsymbol{\alpha}]_{\mathrm{D}} \mathbf{2 0}^{\mathbf{2 0}}=+17.0\left(\mathrm{c}=10 \mathrm{mg} / \mathrm{mL}, \mathrm{CHCl}_{3}\right)$

Appearance: Colorless oil 
Methyl (R,2E,4E)-7-hydroxy-8-methylnona-2,4-dienoate (5n)<smiles>COC(=O)/C=C/C=C/CC(O)C(C)C</smiles>

$20 \mathrm{mg}, 101 \mu \mathrm{mol}, 25 \%$, e.r. $92: 8$, starting from $28.8 \mathrm{mg}$ of isobutyraldehyde.

${ }^{1} \mathbf{H}$ NMR $\left(400 \mathrm{MHz}, \mathrm{C}_{6} \mathrm{D}_{6}\right): \delta[\mathrm{ppm}]=7.42(\mathrm{dd}, 1 \mathrm{H}, J=15.4 \mathrm{~Hz}, 10.8 \mathrm{~Hz}), 5.91-5.82(\mathrm{~m}, 2 \mathrm{H}), 5.76-$ $5.66(\mathrm{~m}, 1 \mathrm{H}), 3.45(\mathrm{~s}, 3 \mathrm{H}), 3.04-2.98(\mathrm{~m}, 1 \mathrm{H}), 1.95-1.85(\mathrm{~m}, 2 \mathrm{H}), 1.44-1.25(\mathrm{~m}, 2 \mathrm{H}), 0.81(\mathrm{~d}, 3 \mathrm{H}, J=$ $6.6 \mathrm{~Hz}), 0.76(\mathrm{~d}, 3 \mathrm{H}, J=6.8 \mathrm{~Hz})$.

${ }^{13}$ C NMR (101 MHz, $\left.\mathrm{C}_{6} \mathrm{D}_{6}\right): \delta[\mathrm{ppm}]=167.2,145.0,141.0,130.8,120.0,75.3,51.1,38.3,33.5,19.0$, 17.2 .

HRMS (EI-quadrupole) m/z: [M- $\left.\mathrm{C}_{4} \mathrm{H}_{8} \mathrm{O}\right]^{+}$Calcd for $\mathrm{C}_{7} \mathrm{H}_{10} \mathrm{O}_{2}$ 126.0681; Found $126.0682^{10}$.

Column chromatography: PE:EtOAc 5:1

$\mathbf{R}_{f}=0.23(\mathrm{PE}: \mathrm{EtOAc} 4: 1)$

$[\boldsymbol{\alpha}]_{\mathrm{D}} \mathbf{2 0}^{\mathbf{2 0}}=+17.0\left(\mathrm{c}=10 \mathrm{mg} / \mathrm{mL}, \mathrm{CHCl}_{3}\right)$

Appearance: Colorless oil

\footnotetext{
${ }^{10}$ We suggest that this fragment originates from a McLafferty-like rearrangement, which takes place between the alcohol moiety and the proximal double bond, and results in the formal loss of isobutyraldehyde
} 
Methyl (R,2E,4E)-7-hydroxy-8,8-dimethylnona-2,4-dienoate (5o)

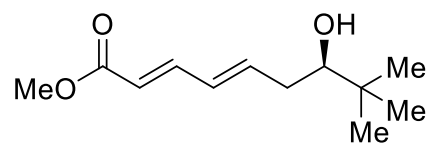

$61 \mathrm{mg}, 287 \mu \mathrm{mol}, 72 \%$, e.r. $95: 5$, starting from $34.4 \mathrm{mg}$ of pivaldehyde.

${ }^{1} \mathbf{H}$ NMR $\left(400 \mathrm{MHz}, \mathrm{C}_{6} \mathrm{D}_{6}\right): \delta[\mathrm{ppm}]=7.45(\mathrm{dd}, 1 \mathrm{H}, J=15.4 \mathrm{~Hz}, 10.8 \mathrm{~Hz}), 5.92-5.84(\mathrm{~m}, 2 \mathrm{H}), 5.81-$ $5.72(\mathrm{~m}, 1 \mathrm{H}), 3.46(\mathrm{~s}, 3 \mathrm{H}), 2.94-2.87(\mathrm{~m}, 1 \mathrm{H}), 2.03-1.95(\mathrm{~m}, 1 \mathrm{H}), 1.87-1.76(\mathrm{~m}, 1 \mathrm{H}), 1.12(\mathrm{br} \mathrm{s}, 1 \mathrm{H})$, $0.81(\mathrm{~s}, 9 \mathrm{H})$.

${ }^{13} \mathrm{C}$ NMR $\left(101 \mathrm{MHz}, \mathrm{C}_{6} \mathrm{D}_{6}\right): \delta[\mathrm{ppm}]=167.3,145.1,142.2,130.7,119.9,78.3,51.1,35.9,34.9,25.8$.

HRMS (EI-quadrupole) m/z: [M- $\left.\mathrm{H}_{2} \mathrm{O}\right]^{+}$Calcd for $\mathrm{C}_{12} \mathrm{H}_{18} \mathrm{O}_{2}$ 194.1307; Found 194.1306.

Column chromatography: PE:EtOAc 10:1

$\mathbf{R}_{f}=0.35$ (PE:EtOAc 4:1)

$[\boldsymbol{\alpha}]_{\mathrm{D}^{20}}=+20.0\left(\mathrm{c}=10 \mathrm{mg} / \mathrm{mL}, \mathrm{CHCl}_{3}\right)$

Appearance: Colorless oil 
Ethyl $(2 E, 4 E, 7 R, 8 R)-9-(($ tert-butyldimethylsilyl)oxy)-7-hydroxy-2,8-dimethylnona-2,4-dienoate (5p)<smiles>[M]C(C[18O])C(O)CC=CC=C(C)C(=O)OCC</smiles>

$106 \mathrm{mg}, 297 \mu \mathrm{mol}, 74 \%$, d.r. $\geq 95: 5$, starting from $81.0 \mathrm{mg}$ of aldehyde $\mathbf{4 n}$.

${ }^{1} \mathbf{H}$ NMR $\left(400 \mathrm{MHz}, \mathrm{C}_{6} \mathrm{D}_{6}\right): \delta[\mathrm{ppm}]=7.47-7.42(\mathrm{~m}, 1 \mathrm{H}), 6.39-6.29(\mathrm{~m}, 1 \mathrm{H}), 5.95-5.85(\mathrm{~m}, 1 \mathrm{H}), 4.07$ (q, 2H, J=7.1 Hz), 3.81-3.74 (m, 1H), 3.50 (d, 2H, J=5.0 Hz), 2.34-2.24 (m, 2H), 2.14-2.04 (m, 1H), 1.96-1.93 (m, 3H), 1.57-1.48 (m, 1H), 1.03 (t, 3H, J=7.1 Hz), 0.92 (s, 9H), 0.88 (2, 3H, J=7.0 Hz), 0.01 $(\mathrm{s}, 6 \mathrm{H})$;

${ }^{13}$ C NMR (101 MHz, $\left.\mathrm{C}_{6} \mathrm{D}_{6}\right): \delta[\mathrm{ppm}]=168.1,139.5,138.5,126.3,72.9,67.7,60.4,39.6,39.1,26.0$, $18.4,14.4,12.9,10.4,-5.4,-5.5$;

HRMS (ESI-TOF) m/z: [M+Na $]^{+}$Calcd for $\mathrm{C}_{19} \mathrm{H}_{36} \mathrm{O}_{4} \mathrm{SiNa} 379.2281$; Found 379.2280.

Column chromatography: PE:EtOAc 10:1 to 5:1

$\mathbf{R}_{f}=0.63$ (PE:EtOAc 4:1)

$[\boldsymbol{\alpha}]_{\mathbf{D}}{ }^{22}=+22.4\left(\mathrm{c}=11.7 \mathrm{mg} / \mathrm{mL}, \mathrm{CHCl}_{3}\right)$

Appearance: Yellow oil 
Ethyl (2E,4E,7R,8S)-9-((tert-butyldimethylsilyl)oxy)-7-hydroxy-2,8-dimethylnona-2,4-dienoate $(\mathbf{5 q})$<smiles>CCOC(=O)C(=CC=CCC(O)C(C[18O])[N+](=O)[O-])C(=O)OC</smiles>

$70 \mathrm{mg}, 196 \mu \mathrm{mol}, 49 \%$, d.r. 90:10, starting from $81.0 \mathrm{mg}$ of aldehyde $4 \mathbf{0 .}$

${ }^{1} \mathbf{H}$ NMR $\left(400 \mathrm{MHz}, \mathrm{CDCl}_{3}\right): \delta[\mathrm{ppm}]=7.22-7.17(\mathrm{~m}, 1 \mathrm{H}), 6.48-6.39(\mathrm{~m}, 1 \mathrm{H}), 6.24-6.16(\mathrm{~m}, 1 \mathrm{H})$, 4.20 (q, 2H, J=7.1 Hz), 3.94 (d, 1H, $J=2.9 \mathrm{~Hz}), 3.80$ (dd, 1H, $J=4.0,10.1 \mathrm{~Hz}), 3.70-3.62(\mathrm{~m}, 1 \mathrm{H}), 3.58$ (dd, 1H, J=7.8, $10.2 \mathrm{~Hz}$ ), 2.53-2.43 (m, 1H), 2.40-2.29 (m, 1H), 1.94-1.91 (m, 3H), 1.80-1.69 (m, 1H), 1.29 (t, 3H, J=7.1 Hz), 0.90 (s, 9H), 0.86 (d, 3H, J=7.0 Hz), 0.08 (s, 6H);

${ }^{13} \mathbf{C}$ NMR $\left(101 \mathrm{MHz}, \mathrm{CDCl}_{3}\right): \delta[\mathrm{ppm}]=168.7,139.1,138.4,128.0,125.5,76.2,68.5,60.5,39.2,39.0$, $25.8,18.1,14.3,13.4,12.6,-5.6,-5.7$;

HRMS (ESI-TOF) m/z: [M+Na] ${ }^{+}$Calcd for $\mathrm{C}_{19} \mathrm{H}_{36} \mathrm{O}_{4} \mathrm{SiNa} 379.2281$; Found 379.2276.

Column chromatography: PE:EtOAc 10:1 to 5:1

$\mathbf{R}_{f}=0.63$ (PE:EtOAc 4:1)

$[\alpha]_{\mathrm{D}}^{22}=+18.3\left(\mathrm{c}=10.9 \mathrm{mg} / \mathrm{mL}, \mathrm{CHCl}_{3}\right)$

Appearance: Yellow oil 
Ethyl (2E,4E,7R,9S)-9-((tert-butyldimethylsilyl)oxy)-7-hydroxy-2-methyldeca-2,4-dienoate (5r)<smiles>CCOC(=O)/C(C)=C/C=C/CC(O)CC(C)OCC</smiles>

$107 \mathrm{mg}, 301 \mu \mathrm{mol}, 75 \%$, d.r. $93: 7$, starting from $81.0 \mathrm{mg}$ of aldehyde $\mathbf{4 p .}$

${ }^{1} \mathbf{H}$ NMR (400 MHz, $\left.\mathrm{CDCl}_{3}\right): \delta[\mathrm{ppm}]=7.20-7.15(\mathrm{~m}, 1 \mathrm{H}), 6.44-6.38(\mathrm{~m}, 1 \mathrm{H}), 6.14-6.07(\mathrm{~m}, 1 \mathrm{H})$, 4.22 (q, 2H, J=7.1 Hz), 4.11-4.05 (m, 1H), 3.91-3.86 (m, 1H), 3.61 (br s), 2.41-2.30 (m, 2H), 1.94-1.91 (m, 3H), 1.60-1.54 (m, 2H), 1.29 (t, 3H, J=7.2 Hz), 1.19 (d, 3H, J=6.1 Hz), 0.90 (s, 9H), 0.12 (s, 3H), $0.11(\mathrm{~s}, 3 \mathrm{H})$.

${ }^{13} \mathrm{C}$ NMR $\left(101 \mathrm{MHz}, \mathrm{CDCl}_{3}\right): \delta[\mathrm{ppm}]=168.7,138.6,138.3,128.5,126.0,71.1,70.3,60.6,45.2,41.5$, $25.9,24.8,18.0,14.5,12.7,-3.7,-4.7$.

HRMS (ESI-TOF) m/z: [M+Na] ${ }^{+}$Calcd for $\mathrm{C}_{19} \mathrm{H}_{36} \mathrm{O}_{4} \mathrm{SiNa} 379.2281$; Found 379.2279.

Column chromatography: PE:EtOAc 10:1 to 5:1

$\mathbf{R}_{f}=0.44$ (PE:EtOAc 4:1)

$[\boldsymbol{\alpha}]_{\mathrm{D}}{ }^{22}=+26.9\left(\mathrm{c}=13.0 \mathrm{mg} / \mathrm{mL}, \mathrm{CHCl}_{3}\right)$

Appearance: Yellow oil 
Ethyl (2E,4E,7R,9R)-9-((tert-butyldimethylsilyl)oxy)-7-hydroxy-2-methyldeca-2,4-dienoate (5s)<smiles>CCOC(=O)/C(=C/C=C/CC(O)CC(C)O[Na])O[Na]</smiles>

$78 \mathrm{mg}, 220 \mu \mathrm{mol}, 55 \%$, d.r. $47: 53^{11}$, starting from $81.0 \mathrm{mg}$ of aldehyde $\mathbf{4 p}$.

${ }^{1} \mathbf{H}$ NMR (400 MHz, $\left.\mathrm{CDCl}_{3}\right): \delta[\mathrm{ppm}]=7.20-7.15(\mathrm{~m}, 1 \mathrm{H}), 6.45-6.38(\mathrm{~m}, 1 \mathrm{H}), 6.14-6.07(\mathrm{~m}, 1 \mathrm{H})$, $4.24-4.21^{*}(\mathrm{~m}, 1 \mathrm{H}), 4.22-4.18(\mathrm{~m}, 2 \mathrm{H}$, major and minor isomer), 4.11-4.05 (m, 2H, major and minor isomer), 3.91-3.86 (m, 1H), 3.61 (br s, 1H), 3.49* (br s, 1H), 2.42-2.28 (m, 2H), 1.94-1.91 (m, 3H), $1.67 *$ (ddd, $1 \mathrm{H}, J=3.9,10.2,14.2 \mathrm{~Hz}$ ), 1.60-1.55 (m, 2H), 1.52* (ddd, 1H, J=2.1, 5.0, $14.3 \mathrm{~Hz}$ ), 1.30 (t, $3 \mathrm{H}, J=7.1 \mathrm{~Hz}), 1.23$ (d, 3H, J=6.7 Hz), 1.18 (d, 3H, J=6.0 Hz), 0.90 (s, 9H), 0.89* (s, 9H), 0.12 (s, 3H), $0.11(\mathrm{~s}, 3 \mathrm{H}), 0.09 *(\mathrm{~s}, 3 \mathrm{H}), 0.085^{*}(\mathrm{~s}, 3 \mathrm{H})$.

${ }^{13} \mathbf{C ~ N M R}\left(101 \mathrm{MHz}, \mathrm{CDCl}_{3}\right): \delta[\mathrm{ppm}]=168.7,138.7^{*}, 138.6,138.3,128.5,128.4^{*}, 125.97^{*}, 125.96$, $71.1,70.3,67.9^{*}, 67.7,60.6,45.3,43.8^{*}, 41.8^{*}, 41.5,25.94,25.93^{*}, 24.8,22.8^{*}, 18.1^{*}, 18.0,14.5,12.8$.

HRMS (ESI-TOF) m/z: [M+Na $]^{+}$Calcd for $\mathrm{C}_{19} \mathrm{H}_{36} \mathrm{O}_{4} \mathrm{SiNa} 379.2281$; Found 379.2285.

Column chromatography: PE:EtOAc 10:1 to 5:1

$\mathbf{R}_{f}=0.44$ (PE:EtOAc 4:1)

Appearance: Yellow oil

${ }^{11}$ The major diastereomer is the syn-diastereomer $\mathbf{5 r}$. 


\section{Ethyl (2E,4E,7R,9S)-7-hydroxy-9-((4-methoxybenzyl)oxy)-2-methyldeca-2,4-dienoate (5t)}<smiles>CCOC(=O)/C(C)=C/C=C/CC(O)CC(C)O[Na]</smiles>

$109 \mathrm{mg}, 301 \mu \mathrm{mol}, 75 \%$, d.r. $76: 24$, starting from $83.2 \mathrm{mg}$ of aldehyde $\mathbf{4 q}$.

${ }^{1} \mathbf{H}$ NMR $\left(400 \mathrm{MHz}, \mathrm{CDCl}_{3}\right): \delta[\mathrm{ppm}]=7.26-7.24(\mathrm{~m}, 2 \mathrm{H}), 7.19-7.13(\mathrm{~m}, 1 \mathrm{H}), 6.90-6.85(\mathrm{~m}, 2 \mathrm{H})$, 6.44-6.35 (m, 1H), 6.13-6.03 (m, 1H), 4.60 (d, 1H, J=11.2 Hz), 4.56* (d, 1H, J=11.3 Hz), 4.38 (d, 1H, $J=11.3 \mathrm{~Hz}), 4.34(\mathrm{~d}, 1 \mathrm{H}, J=11.0 \mathrm{~Hz}), 4.22-4.16(\mathrm{~m}, 2 \mathrm{H}), 4.06-3.98^{*}(\mathrm{~m}, 1 \mathrm{H}), 3.92-3.83(\mathrm{~m}, 1 \mathrm{H})$, 3.82-3.76 (m, 4H), 2.41-2.25 (m, 2H), 1.94-1.90 (m, 3H), 1.73-1.54 (m, 2H), 1.33-1.27 (m, 3H), $1.27 *(\mathrm{~d}, 3 \mathrm{H}, J=6.7 \mathrm{~Hz}), 1.23(\mathrm{~d}, 3 \mathrm{H}, J=6.0 \mathrm{~Hz})$.

${ }^{13} \mathrm{C}$ NMR $\left(101 \mathrm{MHz}, \mathrm{CDCl}_{3}\right): \delta[\mathrm{ppm}]=168.74,168.72^{*}, 159.5,159.4^{*}, 138.6,138.3,138.2^{*}, 130.5^{*}$, 130.1, 129.6, 129.5*, 128.5*, 128.4, 126.0*, 125.9, 114.1, 114.0*, 75.9, 72.3*, 71.4, 70.3*, 70.1, 60.64*, $60.61,55.4,43.4,42.4^{*}, 41.51,41.47,19.8,19.2^{*}, 14.5,12.7$.

HRMS (ESI-TOF) m/z: [M+Na] ${ }^{+}$Calcd for $\mathrm{C}_{21} \mathrm{H}_{30} \mathrm{O}_{5} \mathrm{Na}$ 385.1991; Found 385.1989.

\section{Column chromatography: PE:EtOAc 5:1}

$\mathbf{R}_{f}=0.20($ PE:EtOAc 4:1)

Appearance: Yellow oil 
Ethyl (2E,4E,7R,9R)-7-hydroxy-9-((4-methoxybenzyl)oxy)-2-methyldeca-2,4-dienoate (5u)

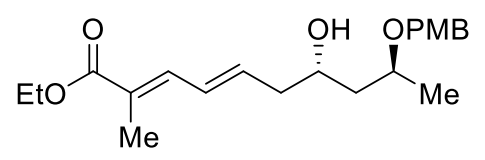

$59 \mathrm{mg}, 164 \mu \mathrm{mol}, 41 \%$, d.r. $84: 16$, starting from $83.2 \mathrm{mg}$ of aldehyde $\mathbf{4 q}$.

${ }^{1} \mathbf{H}$ NMR $\left(600 \mathrm{MHz}, \mathrm{CDCl}_{3}\right): \delta[\mathrm{ppm}]=7.27-7.24(\mathrm{~m}, 2 \mathrm{H}), 7.18-7.15(\mathrm{~m}, 1 \mathrm{H}), 6.89-6.86(\mathrm{~m}, 2 \mathrm{H})$, 6.42-6.36 (m, 1H), 6.12-6.05 (m, 1H), 4.60* (d, 1H, J=10.8 Hz), 4.56 (d, 1H, J=11.0 Hz), 4.38 (d, 1H, $J=11.3 \mathrm{~Hz}), 4.34 *(\mathrm{~d}, 1 \mathrm{H}, J=11.0 \mathrm{~Hz}), 4.20(\mathrm{q}, 2 \mathrm{H}, J=7.2 \mathrm{~Hz}), 4.05-4.00(\mathrm{~m}, 1 \mathrm{H}), 3.92-3.83(\mathrm{~m}, 1 \mathrm{H})$, $3.80(\mathrm{~s}, 3 \mathrm{H}), 2.39-2.27(\mathrm{~m}, 2 \mathrm{H}), 1.93-1.91(\mathrm{~m}, 3 \mathrm{H}), 1.71-1.59$ (m, 2H), 1.30 (t, 3H, J=7.1 Hz), 1.29* $(\mathrm{t}, 3 \mathrm{H}, J=7.1 \mathrm{~Hz}), 1.25$ (d, 3H, $J=6.2 \mathrm{~Hz}), 1.24 *(\mathrm{~d}, 3 \mathrm{H}, J=6.1 \mathrm{~Hz})$.

${ }^{13} \mathbf{C ~ N M R}\left(151 \mathrm{MHz}, \mathrm{CDCl}_{3}\right): \delta[\mathrm{ppm}]=168.7,159.4,138.6,138.2,130.5,129.5,128.5,126.0,114.0$, $72.3,70.3,68.0,60.6,55.4,42.3,41.5,19.2,14.4,12.7$.

HRMS (ESI-TOF) m/z: [M+Na] $]^{+}$Calcd for $\mathrm{C}_{21} \mathrm{H}_{30} \mathrm{O}_{5} \mathrm{Na}$ 385.1991; Found 385.1977.

Column chromatography: PE:EtOAc 5:1

$\mathbf{R}_{f}=0.15$ (PE:EtOAc 4:1)

$[\boldsymbol{\alpha}]_{\mathrm{D}}{ }^{20}=+23.5\left(\mathrm{c}=24.4 \mathrm{mg} / \mathrm{mL}, \mathrm{CHCl}_{3}\right)$

Appearance: Yellow oil 


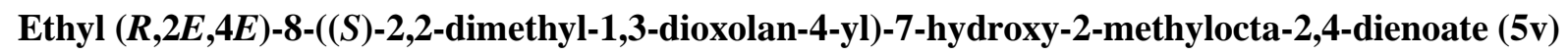

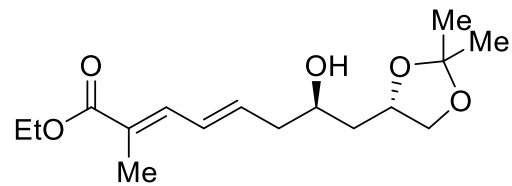

$64 \mathrm{mg}, 215 \mu \mathrm{mol}, 54 \%$, d.r. $\geq 95: 5$, starting from $57.7 \mathrm{mg}$ of aldehyde $\mathbf{4 r}$.

${ }^{1} \mathbf{H}$ NMR (400 MHz, $\left.\mathrm{CDCl}_{3}\right): \delta[\mathrm{ppm}]=7.19-7.13(\mathrm{~m}, 1 \mathrm{H}), 6.49-6.39(\mathrm{~m}, 1 \mathrm{H}), 6.12-6.02(\mathrm{~m}, 1 \mathrm{H})$, 4.39-4.31 (m, 1H), 4.20 (q, 2H, J=7.1 Hz), 4.09 (dd, 1H, J=6.1, 8.1 Hz), 4.01-3.92 (m, 1H), 4.20 (t, $1 \mathrm{H}, J=7.1 \mathrm{~Hz}), 2.46-2.35(\mathrm{~m}, 2 \mathrm{H}), 1.94-1.92(\mathrm{~m}, 3 \mathrm{H}), 1.80-1.67(\mathrm{~m}, 2 \mathrm{H}), 1.42(\mathrm{~s}, 3 \mathrm{H}), 1.36(\mathrm{~s}, 3 \mathrm{H})$, $1.30(\mathrm{t}, 3 \mathrm{H}, J=7.1 \mathrm{~Hz})$.

${ }^{13} \mathbf{C}$ NMR (101 MHz, $\left.\mathrm{CDCl}_{3}\right): \delta[\mathrm{ppm}]=168.7,137.9,137.7,129.1,126.5,109.1,73.6,69.5,68.4,60.7$, $41.7,39.6,27.0,25.8,14.5,12.8$.

HRMS (ESI-TOF) m/z: [M+Na] ${ }^{+}$Calcd for $\mathrm{C}_{16} \mathrm{H}_{26} \mathrm{O}_{5} \mathrm{Na}$ 321.1678; Found 321.1668.

Column chromatography: PE:EtOAc 5:1

$\mathbf{R}_{f}=0.19($ PE:EtOAc 2:1)

$[\alpha]_{\mathrm{D}}{ }^{20}=+0.95(\mathrm{c}=10.5 \mathrm{mg} / \mathrm{mL})$

Appearance: Yellow oil 


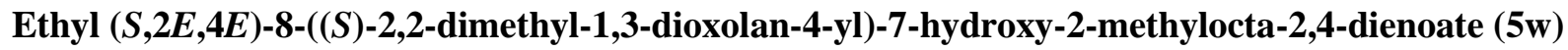

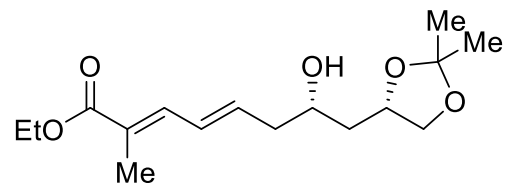

$43 \mathrm{mg}, 144 \mu \mathrm{mol}, 36 \%$, d.r. $\geq 95: 5$, starting from $57.7 \mathrm{mg}$ of aldehyde $\mathbf{4 r}$.

${ }^{1} \mathbf{H}$ NMR $\left(400 \mathrm{MHz}, \mathrm{CDCl}_{3}\right): \delta[\mathrm{ppm}]=7.19-7.13(\mathrm{~m}, 1 \mathrm{H}), 6.46-6.37(\mathrm{~m}, 1 \mathrm{H}), 6.14-6.03(\mathrm{~m}, 1 \mathrm{H})$, 4.31-4.23 (m, 1H), 4.19 (q, 2H, $J=7.1 \mathrm{~Hz}), 4.09$ (dd, $1 \mathrm{H}, J=8.1,6.0 \mathrm{~Hz}), 3.98-3.90(\mathrm{~m}, 2 \mathrm{H}), 3.56$ $(\mathrm{dd}, 1 \mathrm{H}, J=8.1,7.2 \mathrm{~Hz}), 2.46-2.30(\mathrm{~m}, 2 \mathrm{H}), 1.92-1.91(\mathrm{~m}, 3 \mathrm{H}), 1.76-1.58(\mathrm{~m}, 2 \mathrm{H}), 1.42(\mathrm{~s}, 3 \mathrm{H}), 1.36$ $(\mathrm{s}, 3 \mathrm{H}), 1.29(\mathrm{t}, 3 \mathrm{H}, J=7.1 \mathrm{~Hz})$.

${ }^{13} \mathbf{C}$ NMR $\left(101 \mathrm{MHz}, \mathrm{CDCl}_{3}\right): \delta[\mathrm{ppm}]=168.7,138.08,138.0,128.7,126.2,109.7,75.9,70.6,69.8$, $60.7,41.3,39.9,31.0,27.0,25.9,14.4,12.8$.

HRMS (ESI-TOF) m/z: [M+Na] $]^{+}$Calcd for $\mathrm{C}_{16} \mathrm{H}_{26} \mathrm{O}_{5} \mathrm{Na} 321.1678$; Found 321.1682.

Column chromatography: PE:EtOAc 5:1

$\mathbf{R}_{f}=0.32$ (PE:EtOAc 2:1)

$[\boldsymbol{\alpha}]_{\mathbf{D}}{ }^{20}=+4.39\left(\mathrm{c}=11.4 \mathrm{mg} / \mathrm{mL}, \mathrm{CHCl}_{3}\right)$

Appearance: Yellow oil 


\section{General procedure 3: Synthesis of Mosher esters using the Mosher's acid chloride}

4-DMAP (6.00 equiv), $\mathrm{Et}_{3} \mathrm{~N}$ (10.0 equiv) and (S)-MTPA-Cl (6.00 equiv) were added to a solution of the corresponding VMAR-product (usually 20-40 $\mu$ mol, 1.00 equiv) in $\mathrm{CH}_{2} \mathrm{Cl}_{2}(0.5 \mathrm{~mL})$ at r.t. The reaction mixture was stirred for $10 \mathrm{~min}$, then diluted with MTBE. The solution was washed with $\mathrm{NaOH}(1 \mathrm{M}$, $3 \mathrm{x}), \mathrm{NaHCO}_{3}(3 \mathrm{x}), \mathrm{CuSO}_{4}(1 \mathrm{x})$ and brine $(1 \mathrm{x})$, dried over $\mathrm{Na}_{2} \mathrm{SO}_{4}$, and the solvent was removed under reduced pressure. The crude Mosher esters were used for e.r. determination via ${ }^{19} \mathrm{~F}-\mathrm{NMR}$.

\section{Representative Mosher esters for determination of absolute stereochemistry}
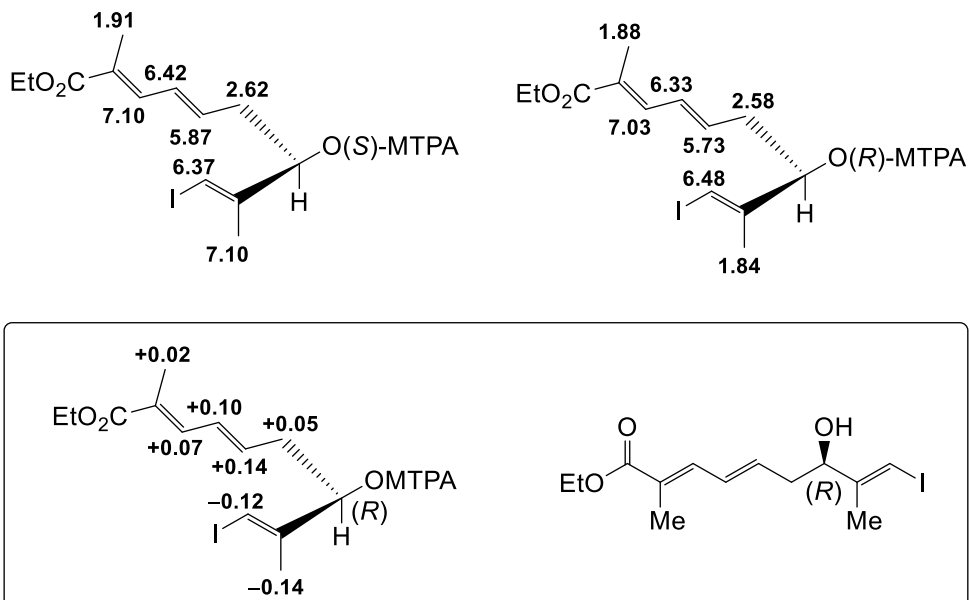

Figure 1. Mosher's ester analysis for the determination of the absolute configuration of bVMAR product $5 \mathrm{~g}$.
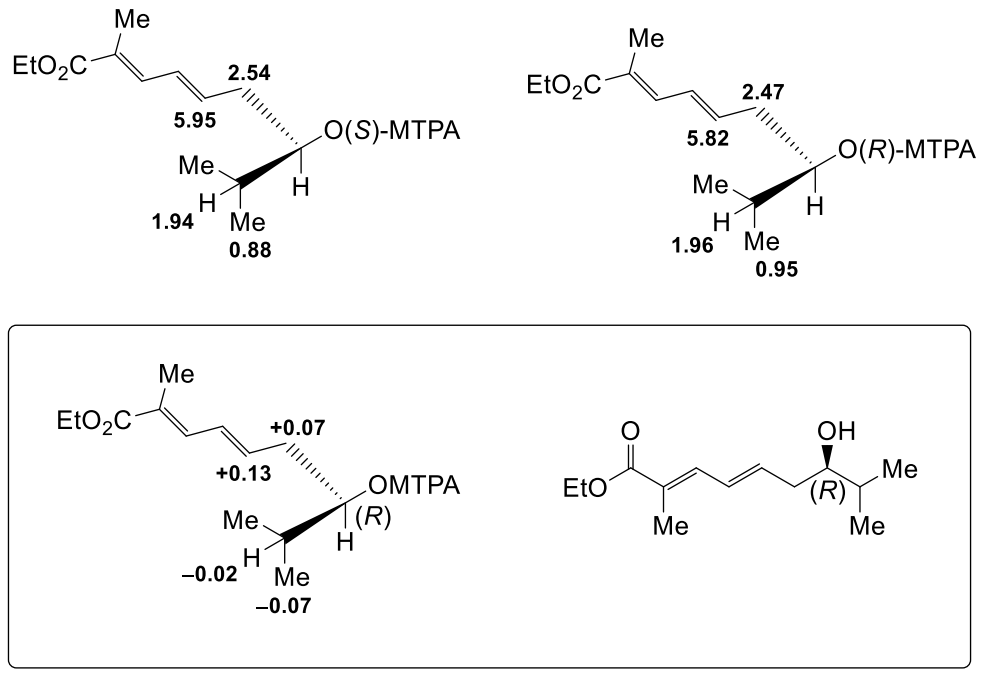

Figure 2. Mosher's ester analysis for the determination of the absolute configuration of bVMAR product $\mathbf{5 j}$. 

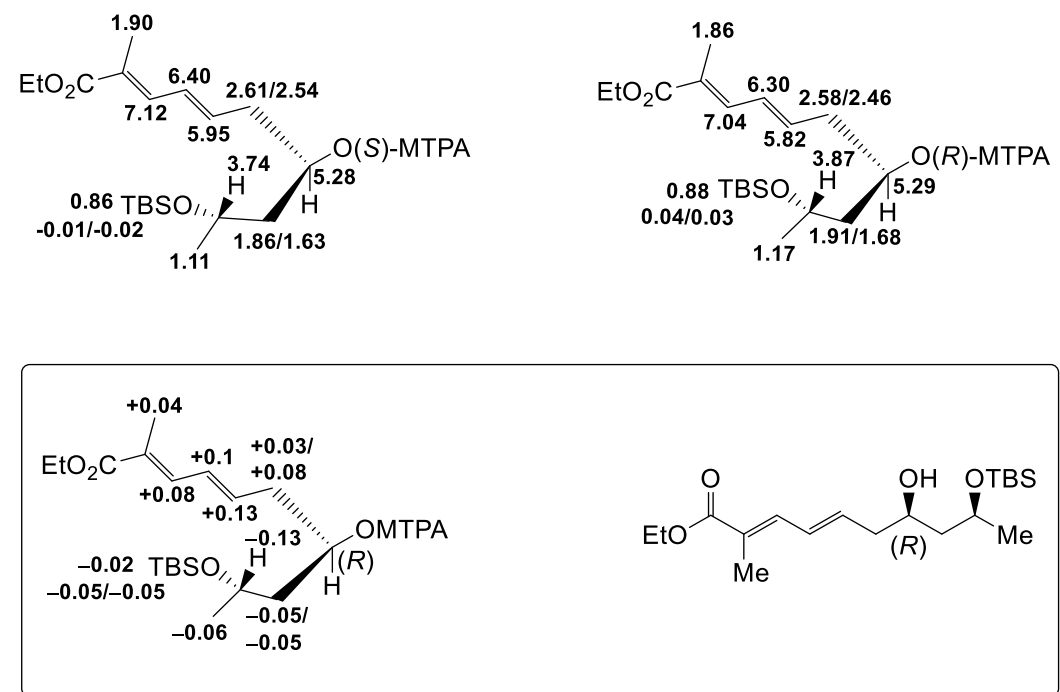

Figure 3. Mosher's ester analysis for the determination of the absolute configuration of bVMAR product $5 \mathbf{r}$.
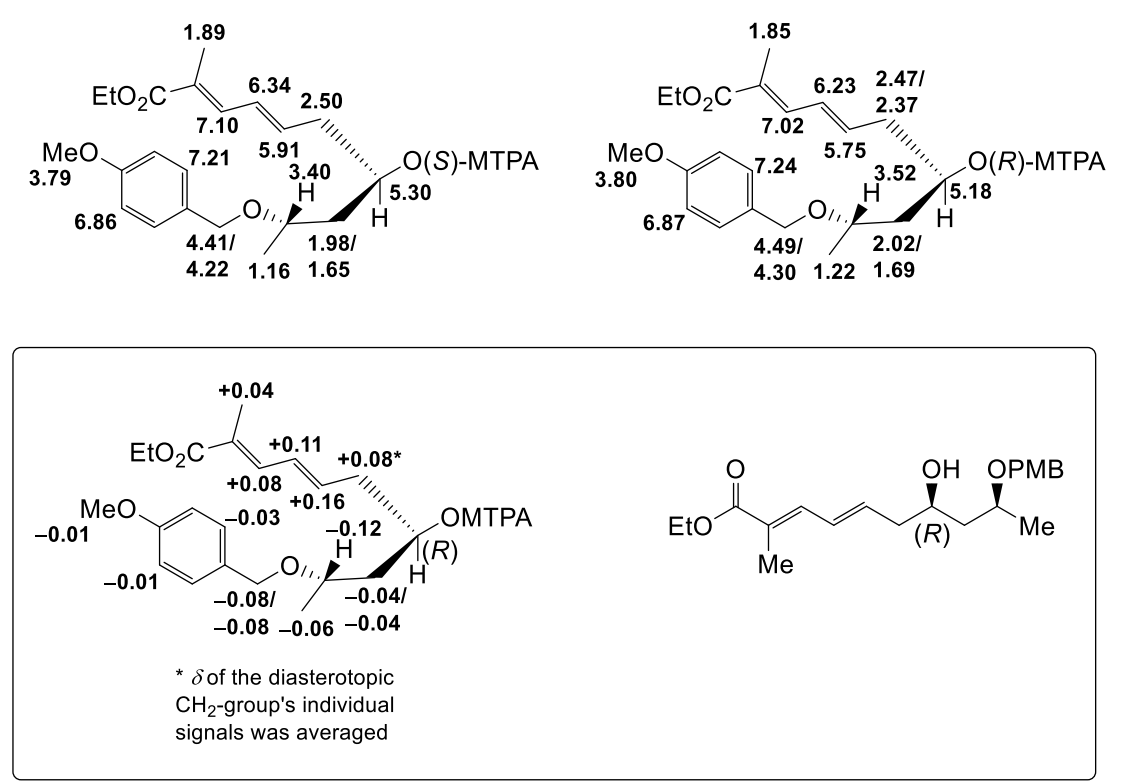

Figure 4. Mosher's ester analysis for the determination of the absolute configuration of bVMAR product 5 t. 

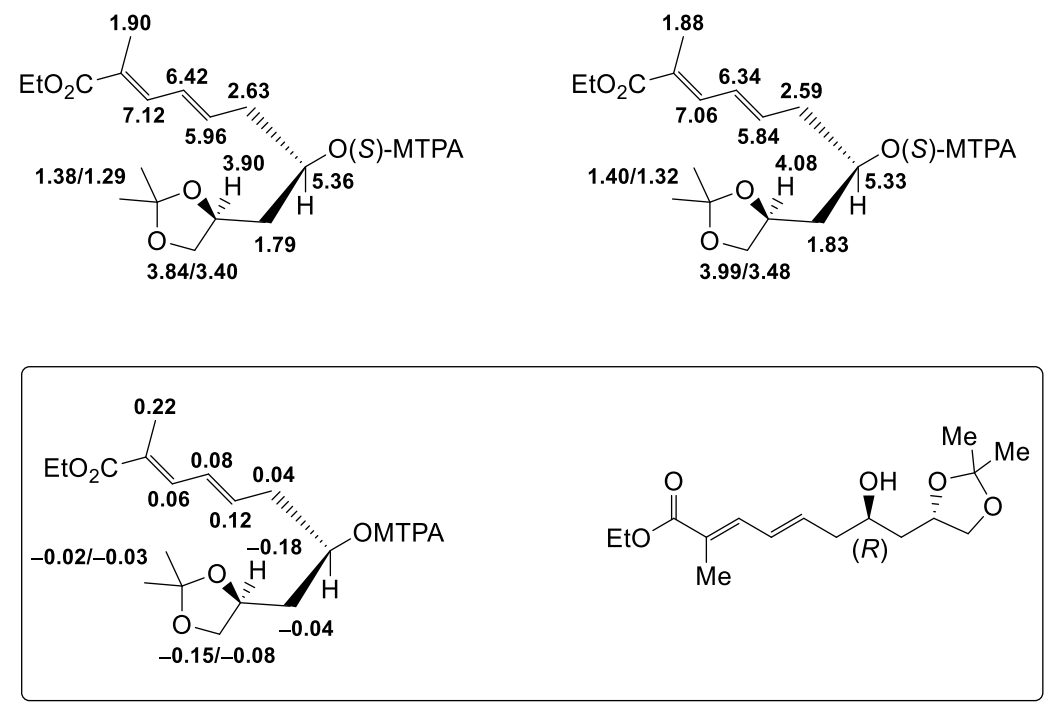

Figure 5. Mosher's ester analysis for the determination of the absolute configuration of bVMAR product $5 \mathbf{v}$. 
Investigations on the substrate-induced stereocontrol of aldehyde $4 \mathrm{p}$ and matched/mismatched cases

Table 1. Lewis acid-dependent distribution syn:anti product distribution for the bVMAR between ketene acetal 3a and $\beta$-chiral aldehyde 4p.

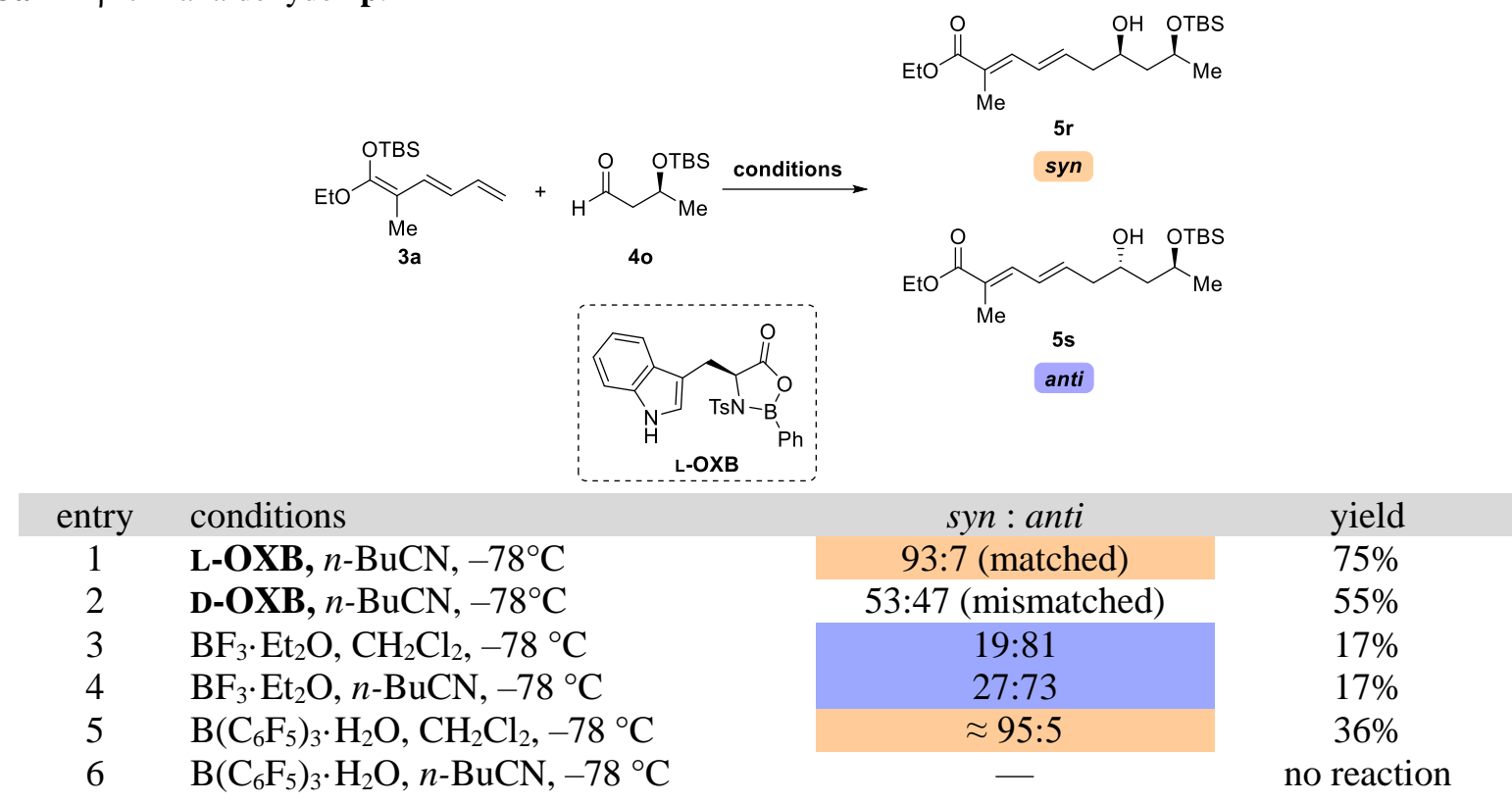


NMR Spectra of new compounds

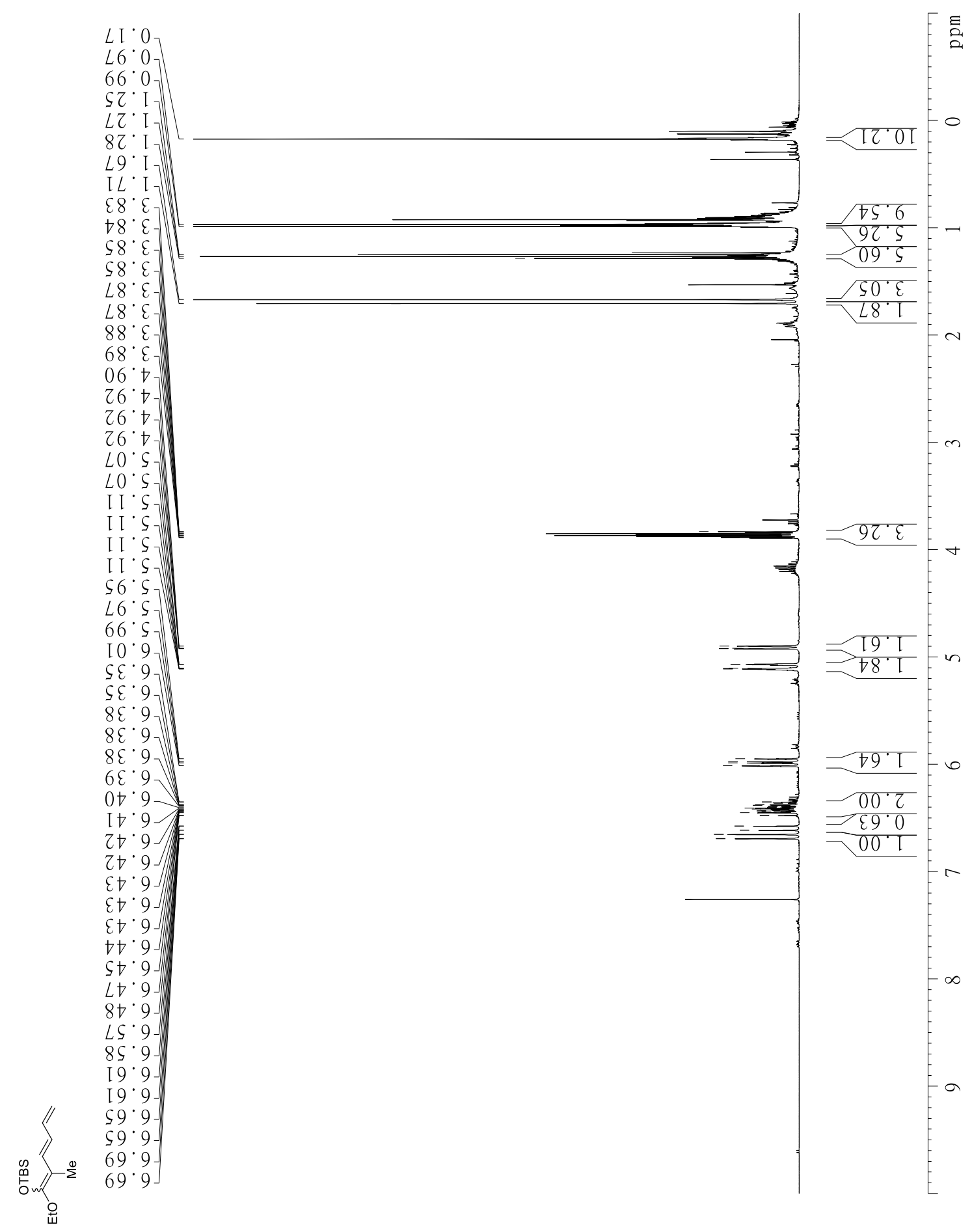

Spectrum 1. ${ }^{1} \mathrm{H}$ spectrum of 3a measured in $\mathrm{CDCl}_{3}$ at $400 \mathrm{MHz}$. 


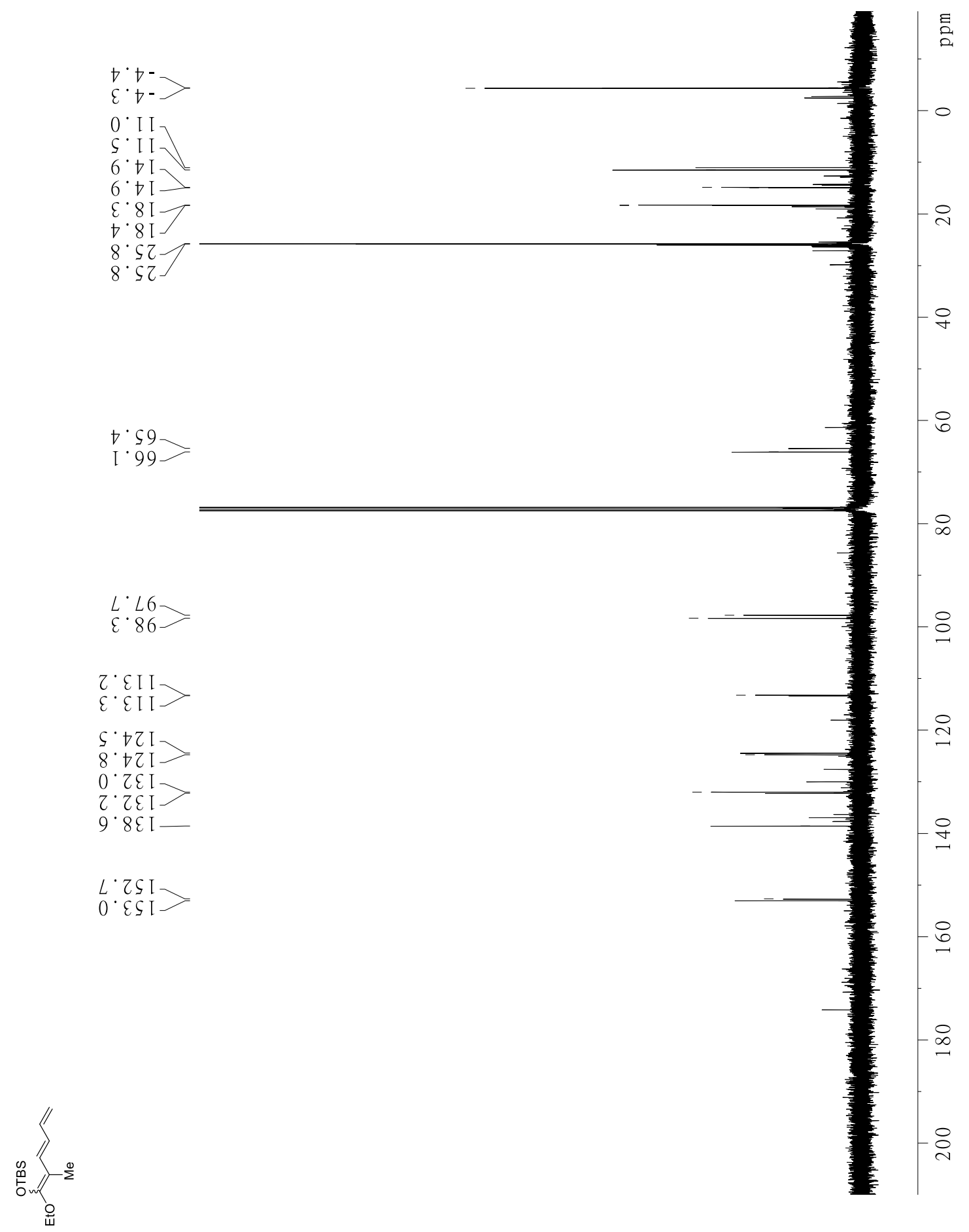

Spectrum 2. ${ }^{13} \mathrm{C}$ spectrum of 3a measured in $\mathrm{CDCl}_{3}$ at $101 \mathrm{MHz}$. 


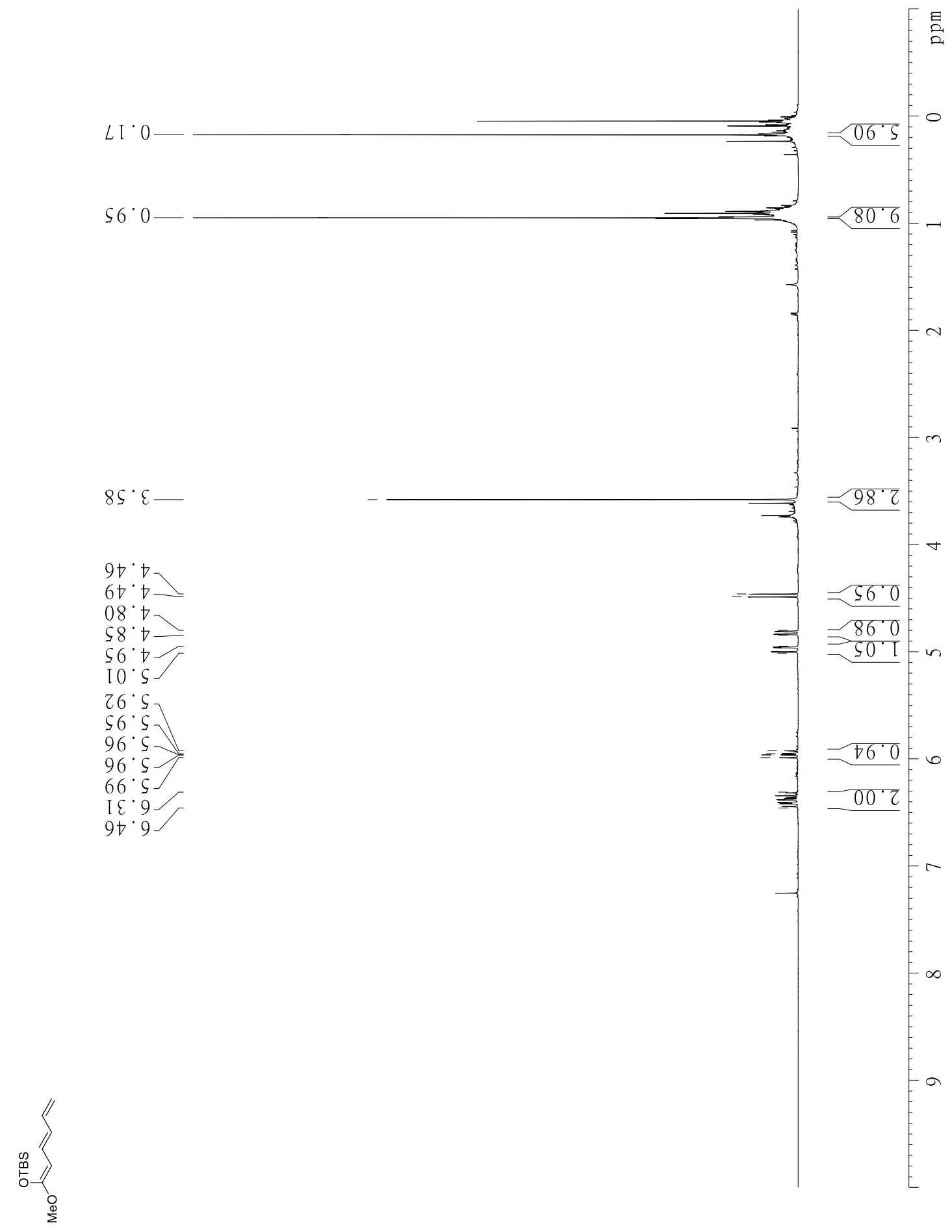

Spectrum 3. ${ }^{1} \mathrm{H}$ spectrum of $3 \mathbf{e}$ measured in $\mathrm{CDCl}_{3}$ at $400 \mathrm{MHz}$. 


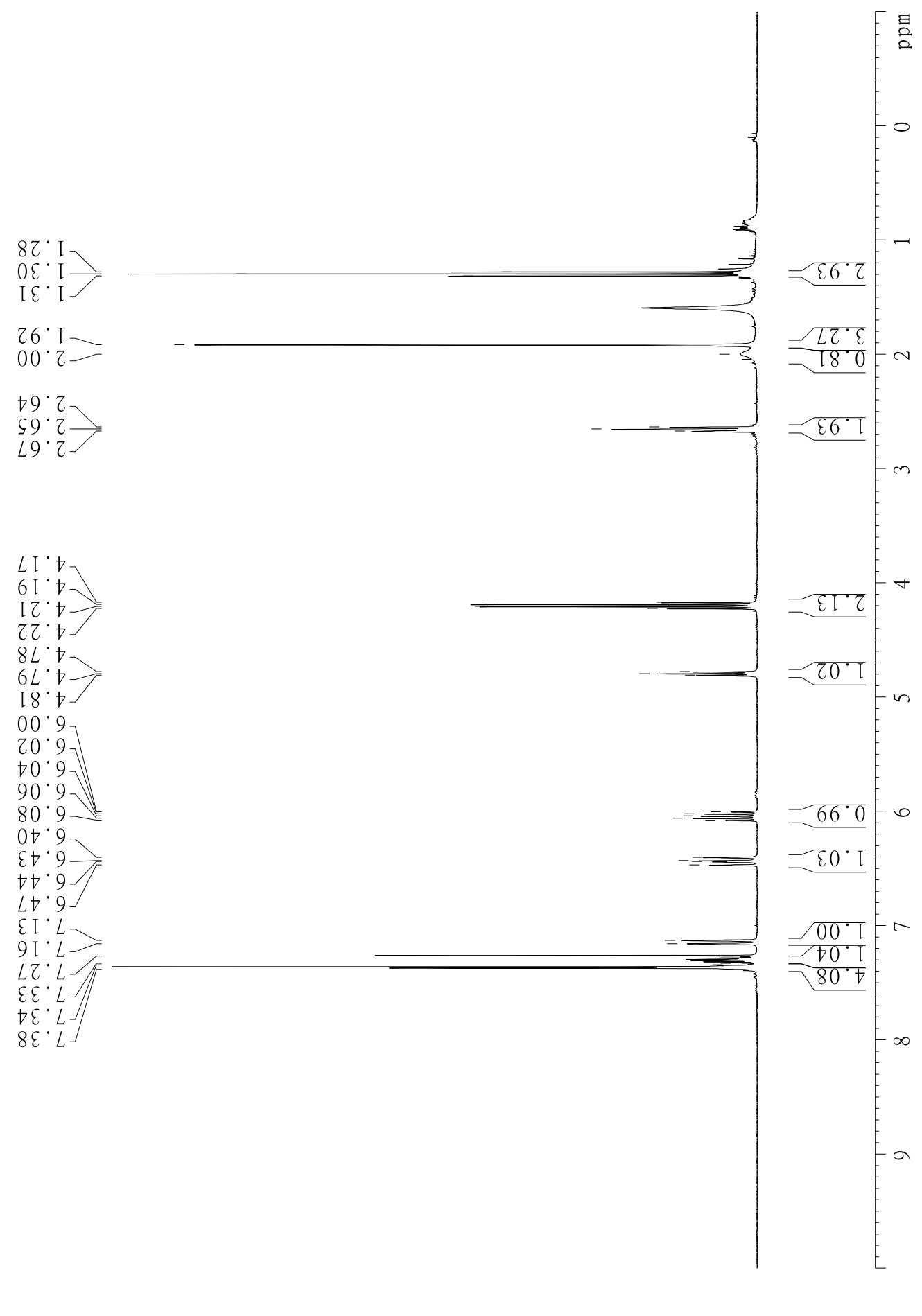

Spectrum 4. ${ }^{1} \mathrm{H}$ spectrum of $5 \mathrm{a}$ measured in $\mathrm{CDCl}_{3}$ at $400 \mathrm{MHz}$. 


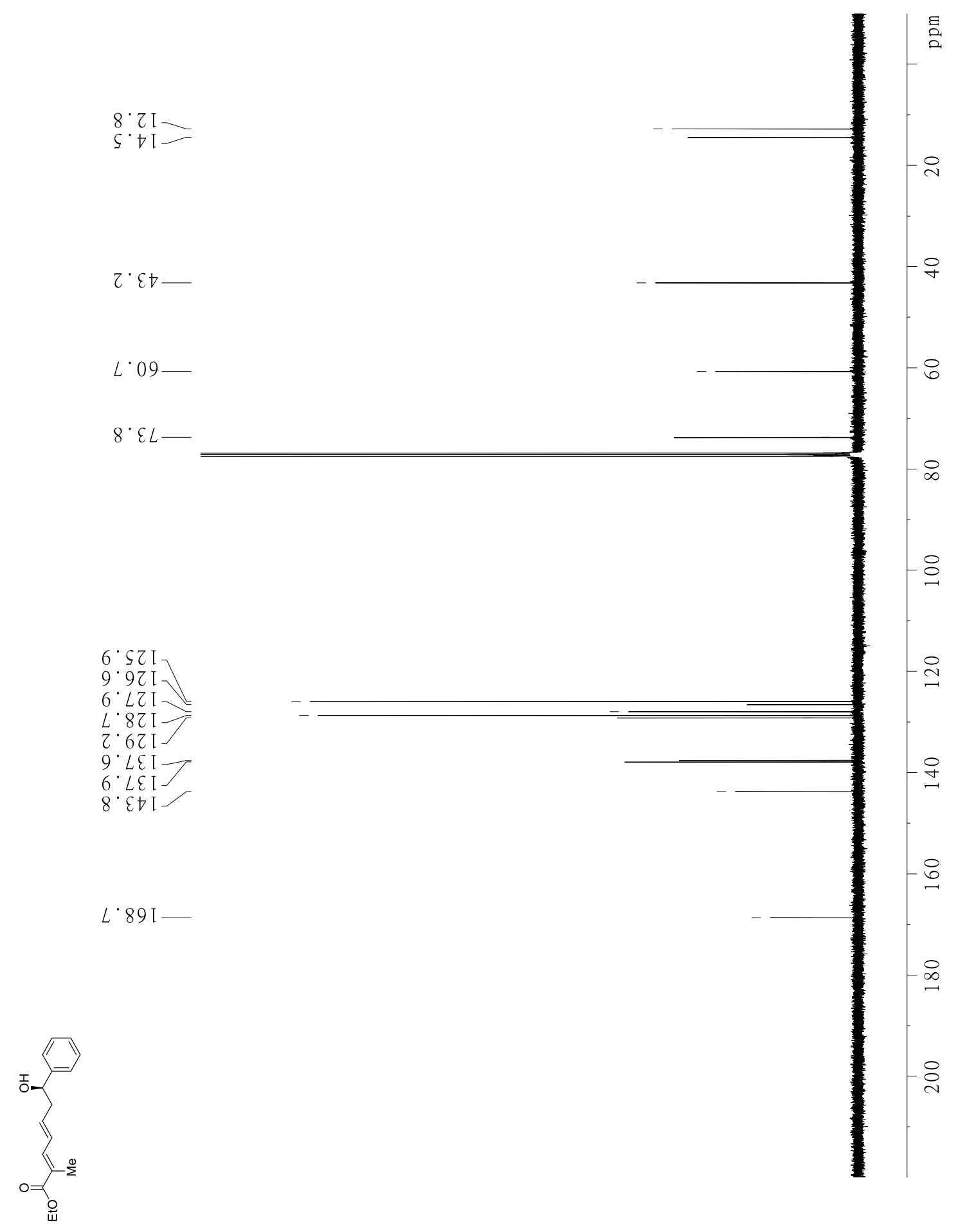

Spectrum 5. ${ }^{13} \mathrm{C}$ spectrum of 5a measured in $\mathrm{CDCl}_{3}$ at $101 \mathrm{MHz}$. 

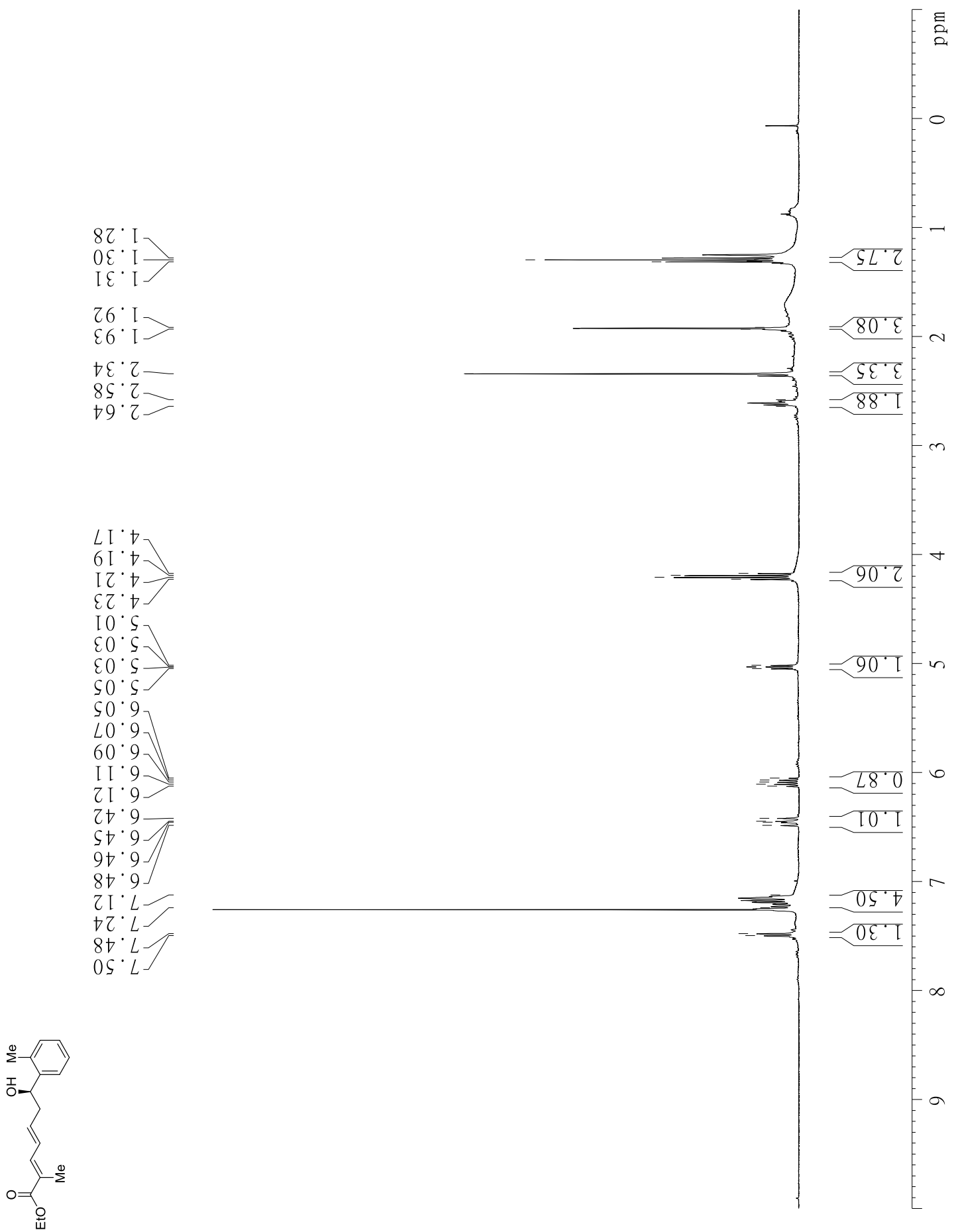


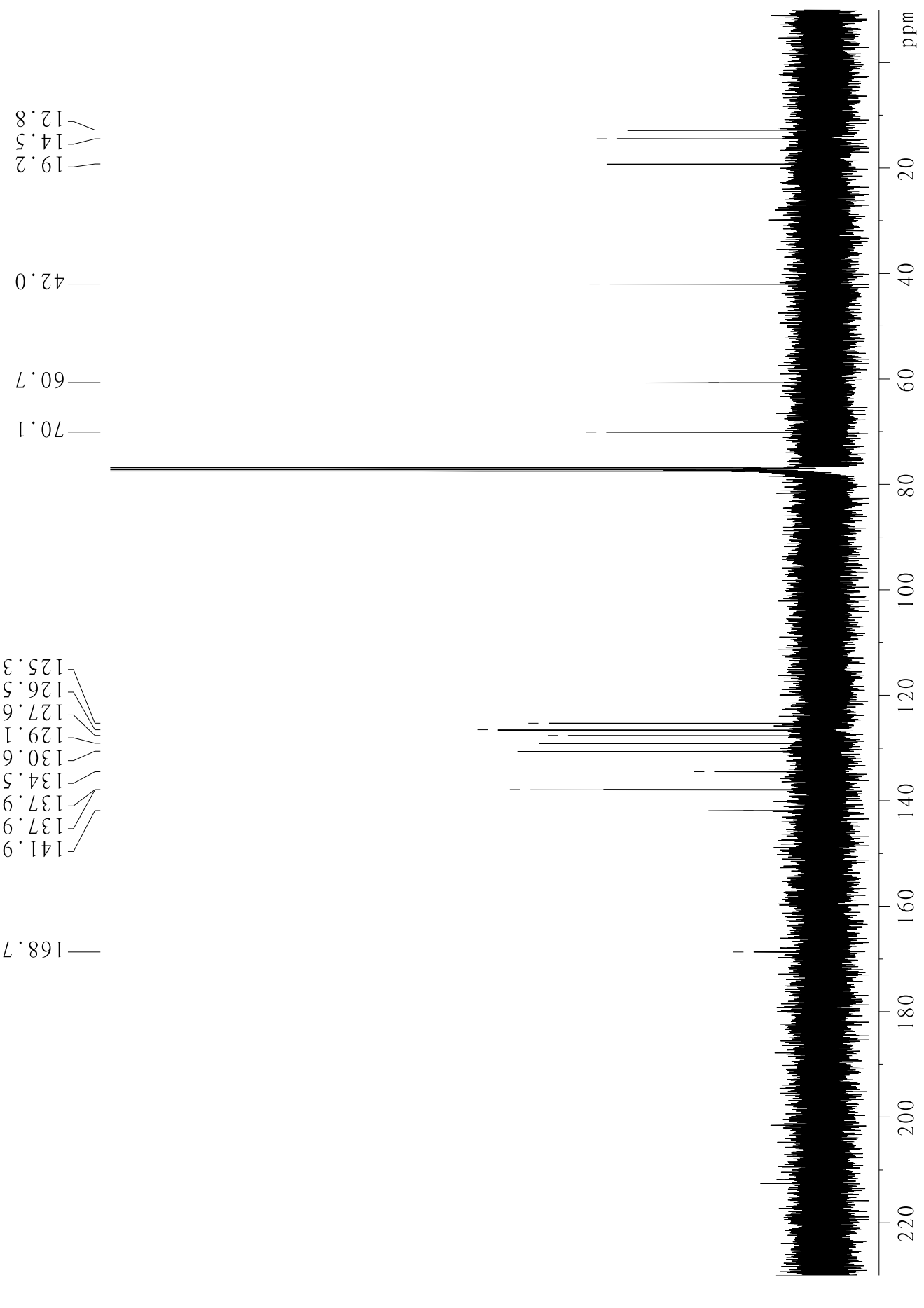

Spectrum 7. ${ }^{13} \mathrm{C}$ spectrum of $\mathbf{5 b}$ measured in $\mathrm{CDCl}_{3}$ at $101 \mathrm{MHz}$. 


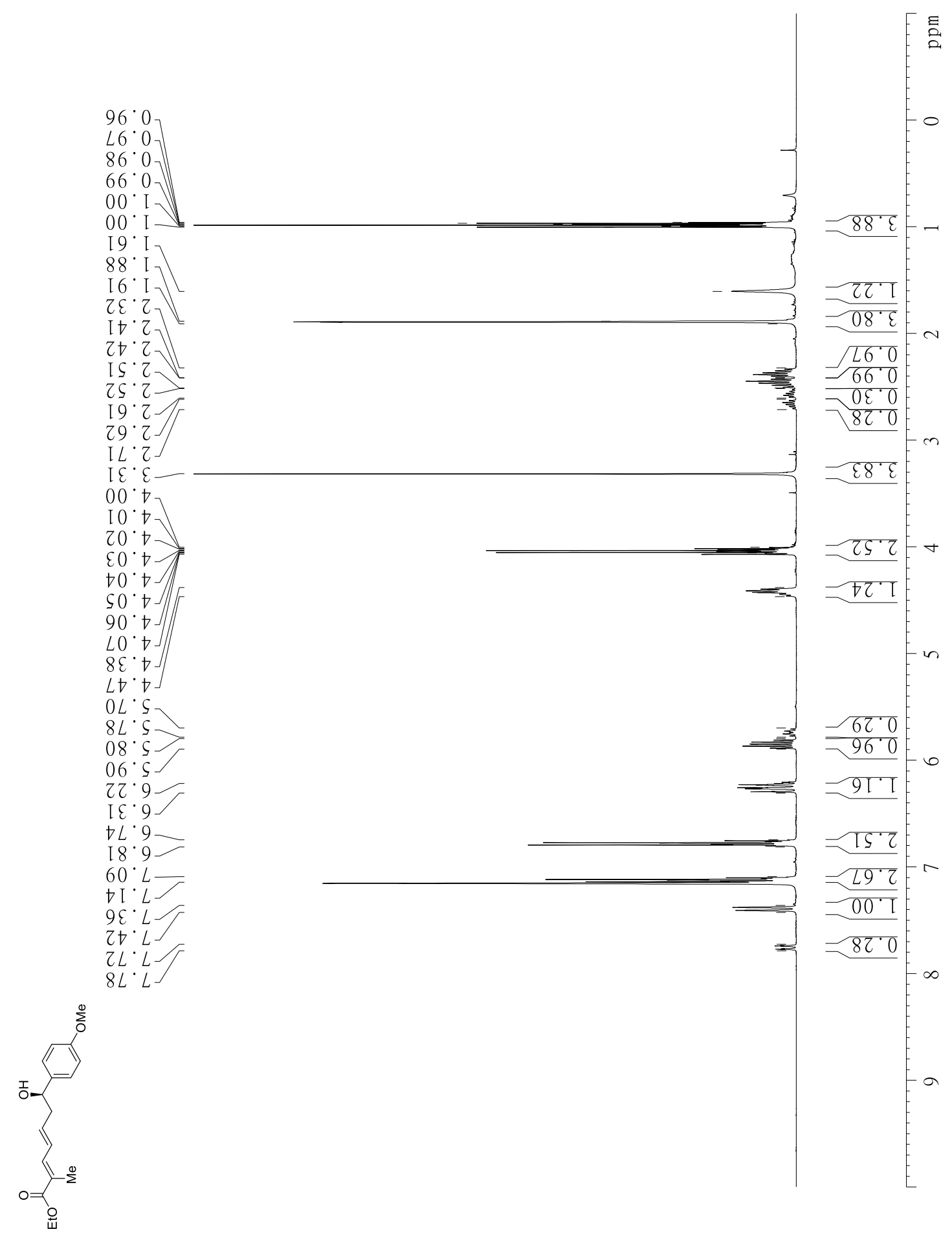

Spectrum 8. ${ }^{1} \mathrm{H}$ spectrum of $\mathbf{5 c}$ measured in $\mathrm{C}_{6} \mathrm{D}_{6}$ at $400 \mathrm{MHz}$. 


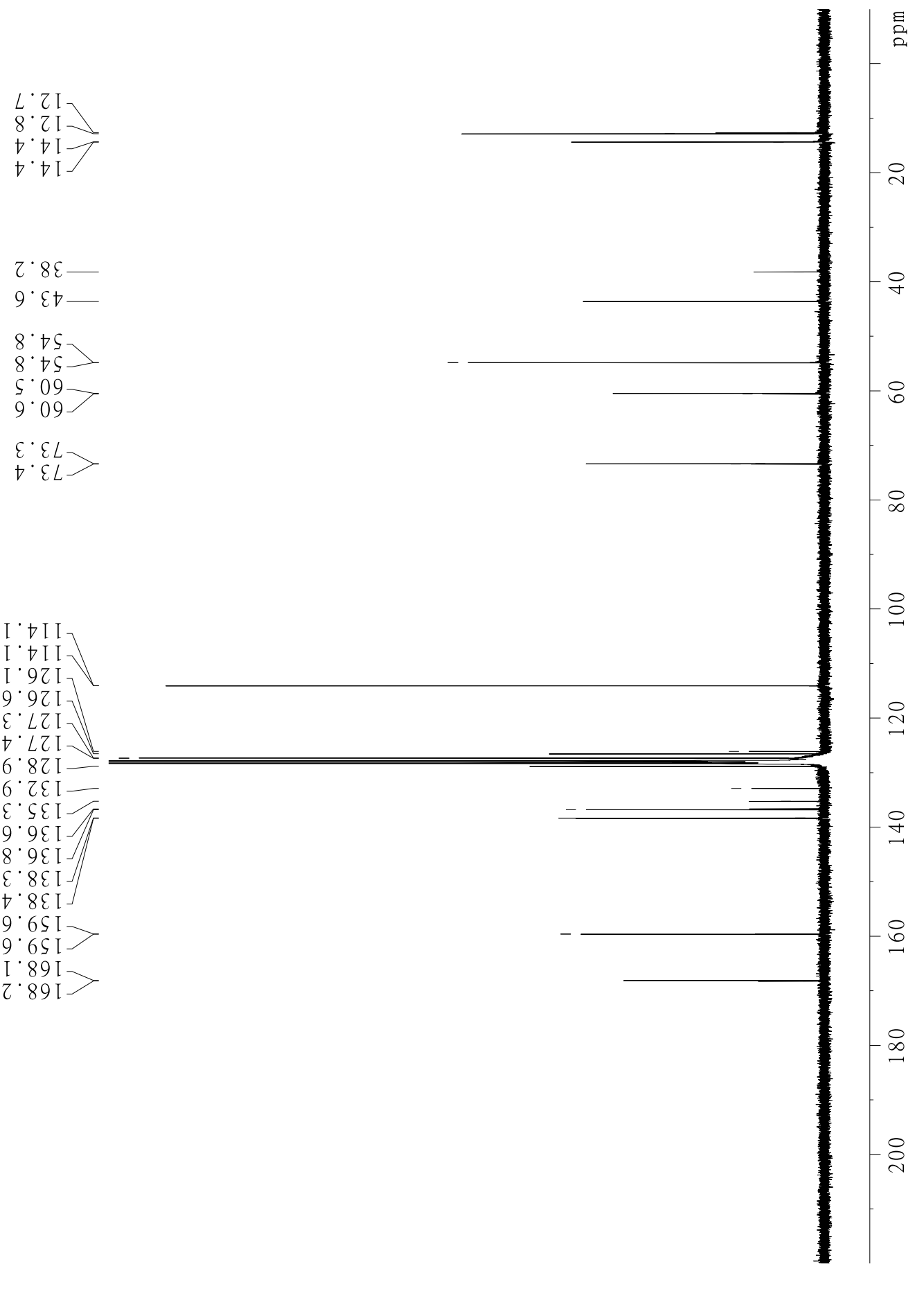

Spectrum 9. ${ }^{13} \mathrm{C}$ spectrum of $\mathbf{5 c}$ measured in $\mathrm{C}_{6} \mathrm{D}_{6}$ at $101 \mathrm{MHz}$. 


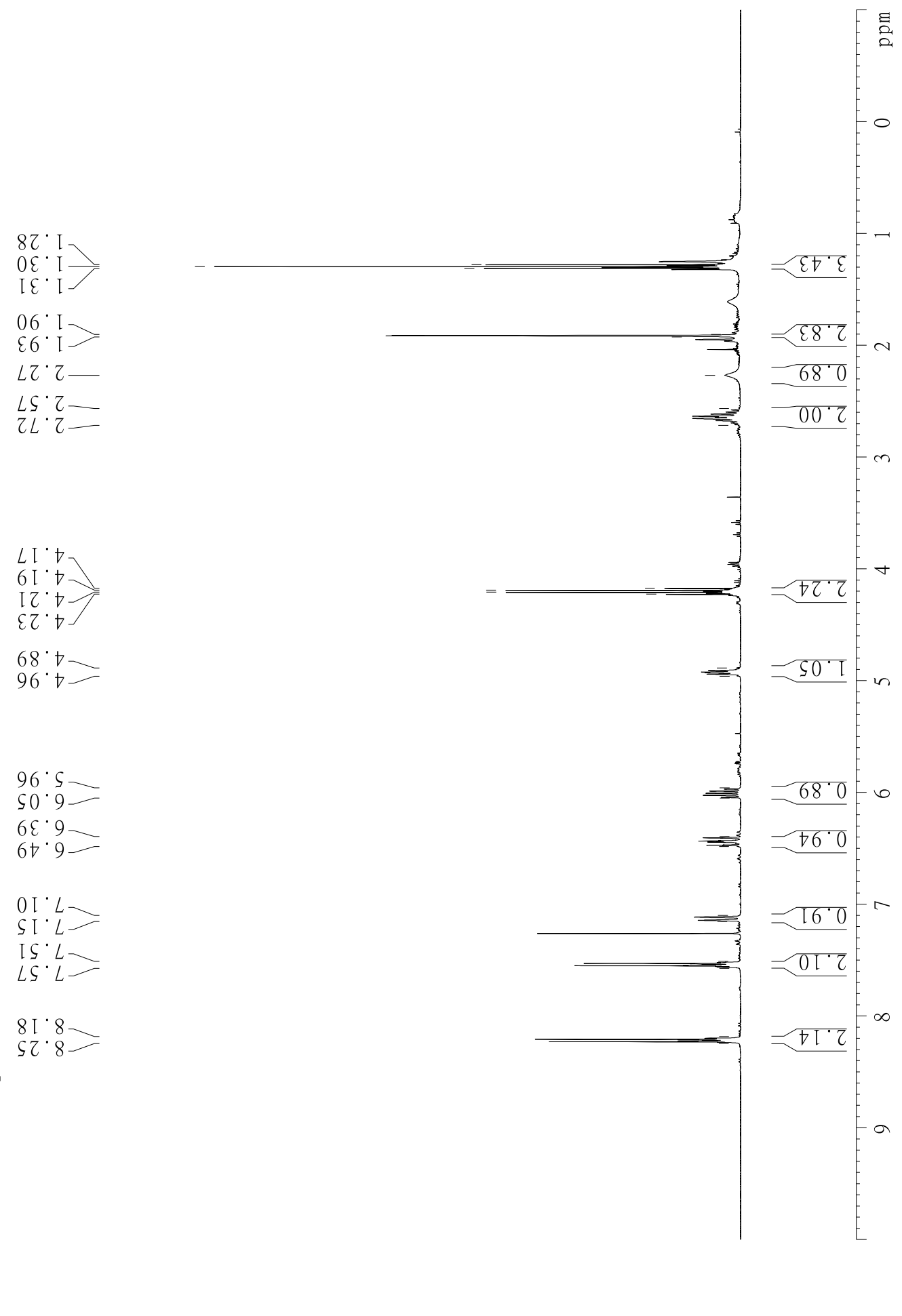

Spectrum 10. ${ }^{1} \mathrm{H}$ spectrum of $\mathbf{5 d}$ measured in $\mathrm{CDCl}_{3}$ at $400 \mathrm{MHz}$. 
$8^{\cdot} \mathrm{ZI}$

$\rightarrow \cdot \mathrm{I}$

$\varepsilon \cdot \varepsilon \nabla$

8.09

$L \cdot T L$

$6 \cdot \varepsilon Z \mathrm{I}$

$L \cdot 92 \mathrm{I}$

$\varepsilon \cdot L Z I$

I. $0 \varepsilon \mathrm{I}$

$6 \cdot \varsigma \varepsilon \mathrm{I}$

$\varepsilon \cdot L \varepsilon I$

$\varsigma \cdot L \triangleright I$

$0^{*}$ IS I -

S.89I

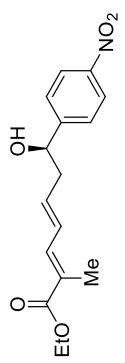

Spectrum 11. ${ }^{13} \mathrm{C}$ spectrum of $\mathbf{5 d}$ measured in $\mathrm{CDCl}_{3}$ at $101 \mathrm{MHz}$. 


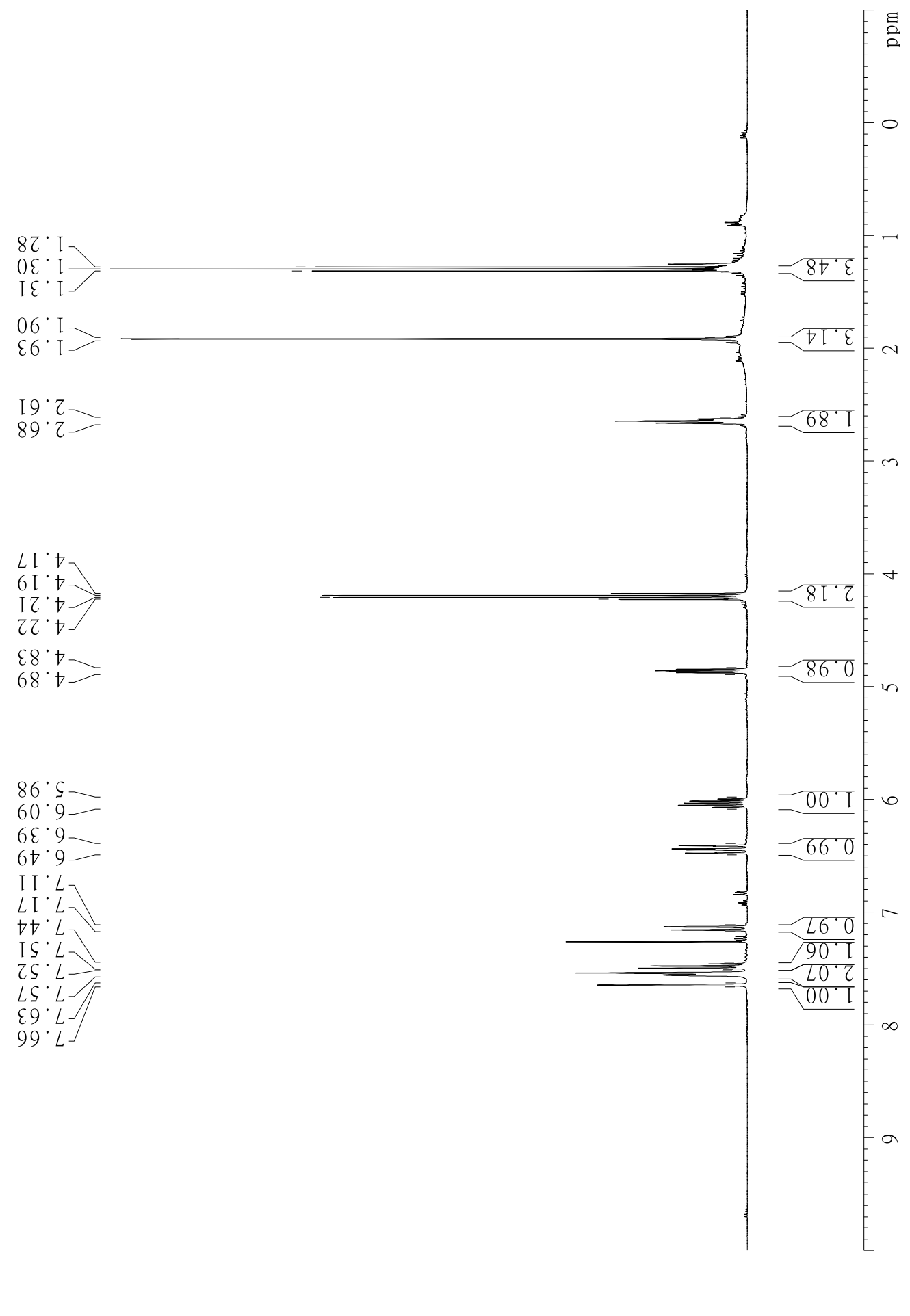

Spectrum 12. ${ }^{1} \mathrm{H}$ spectrum of $\mathbf{5 e}$ measured in $\mathrm{CDCl}_{3}$ at $400 \mathrm{MHz}$. 


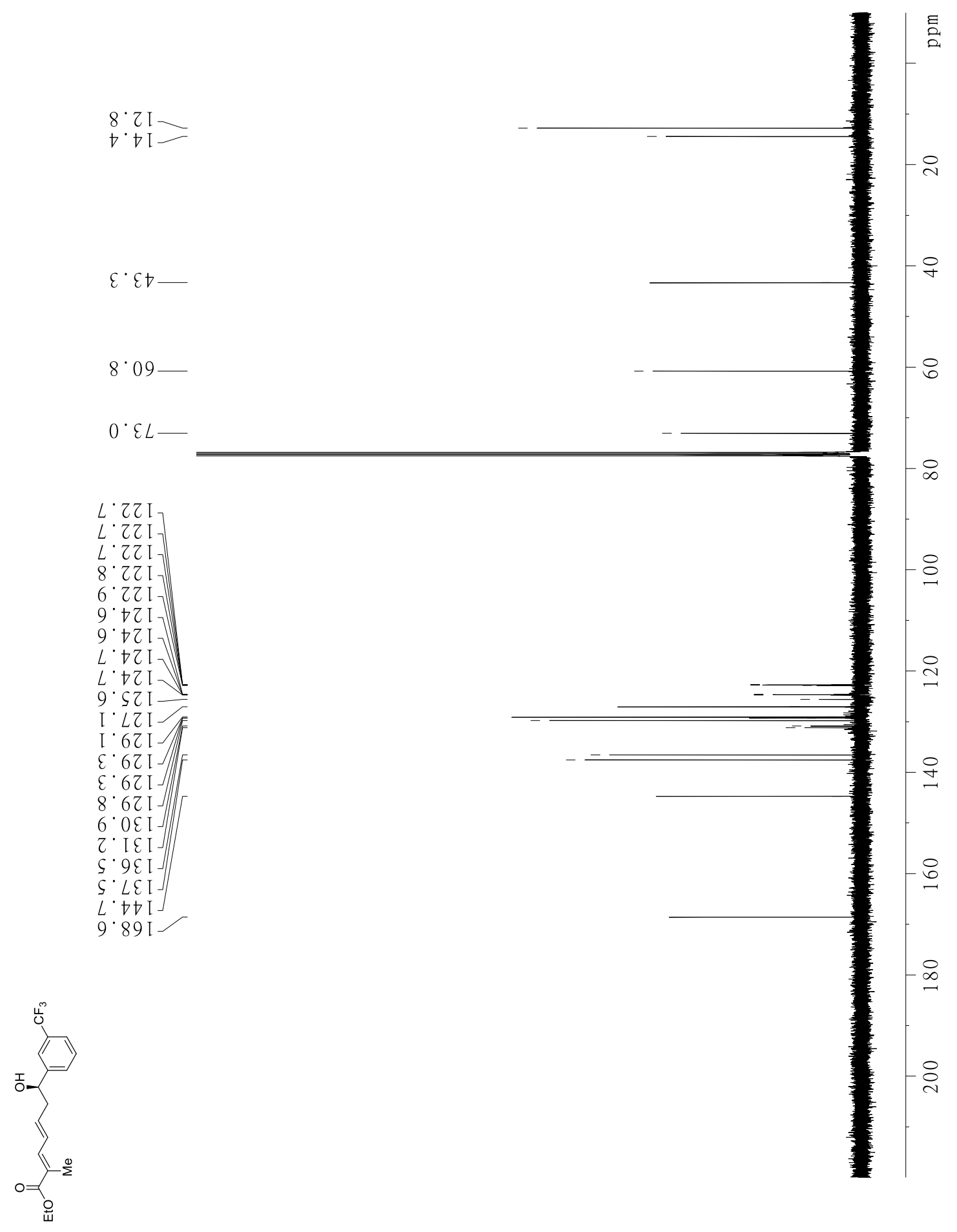

Spectrum 13. ${ }^{13} \mathrm{C}$ spectrum of $\mathbf{5 e}$ measured in $\mathrm{CDCl}_{3}$ at $101 \mathrm{MHz}$. 


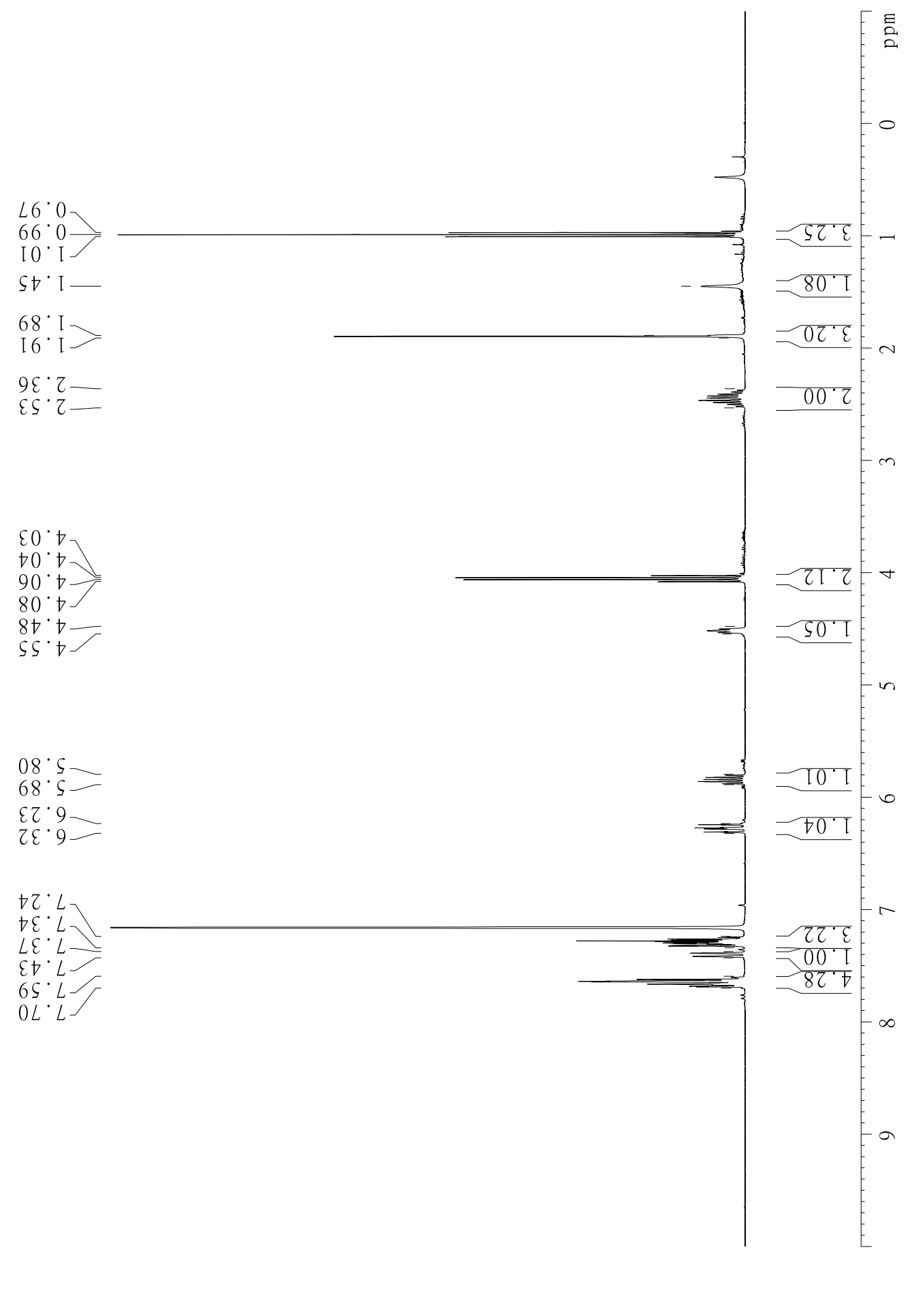

Spectrum 14. ${ }^{1} \mathrm{H}$ spectrum of $\mathbf{5 f}$ measured in $\mathrm{C}_{6} \mathrm{D}_{6}$ at $400 \mathrm{MHz}$. 


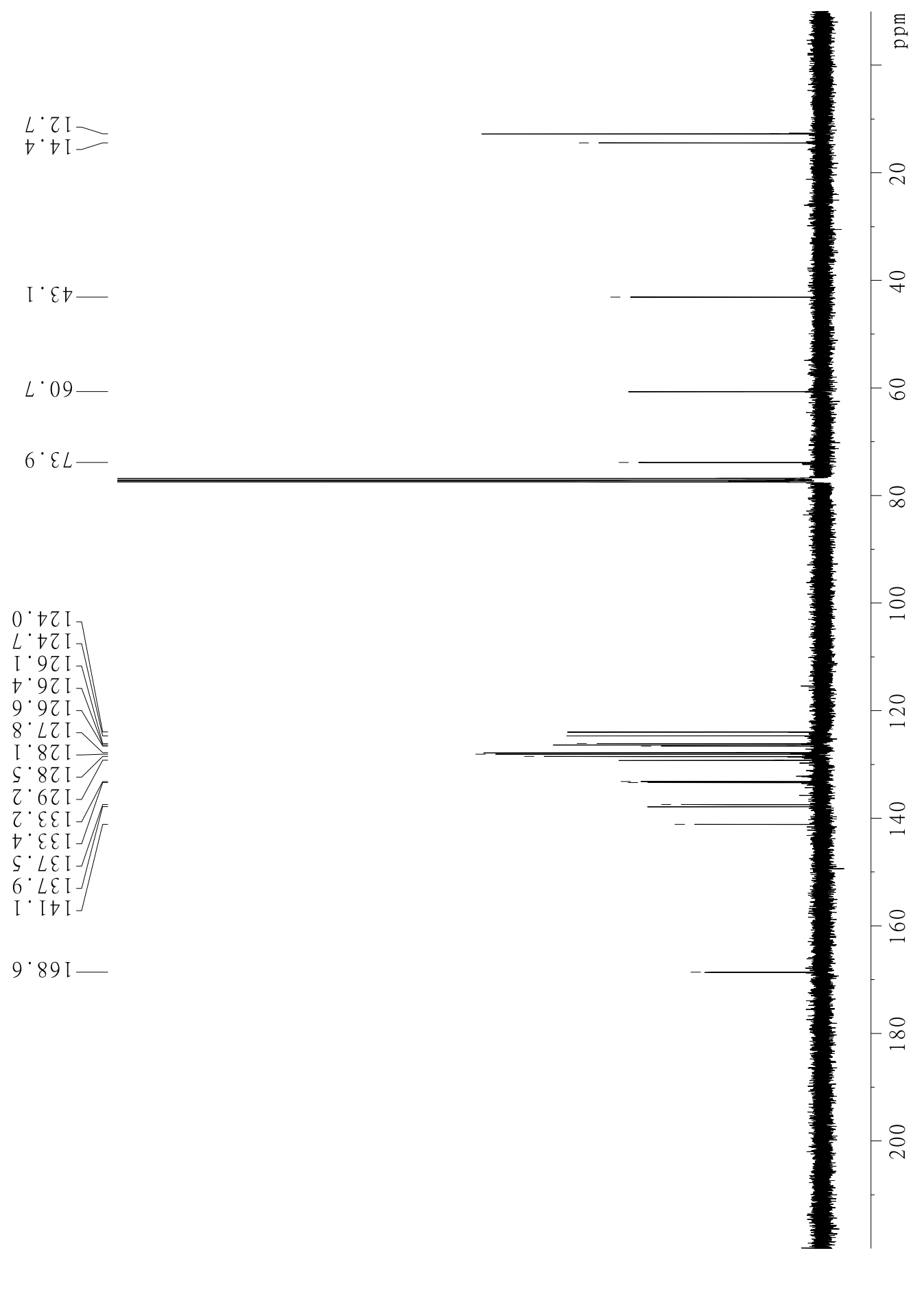

Spectrum 15. ${ }^{13} \mathrm{C}$ spectrum of $\mathbf{5 f}$ measured in $\mathrm{CDCl}_{3}$ at $101 \mathrm{MHz}$. 


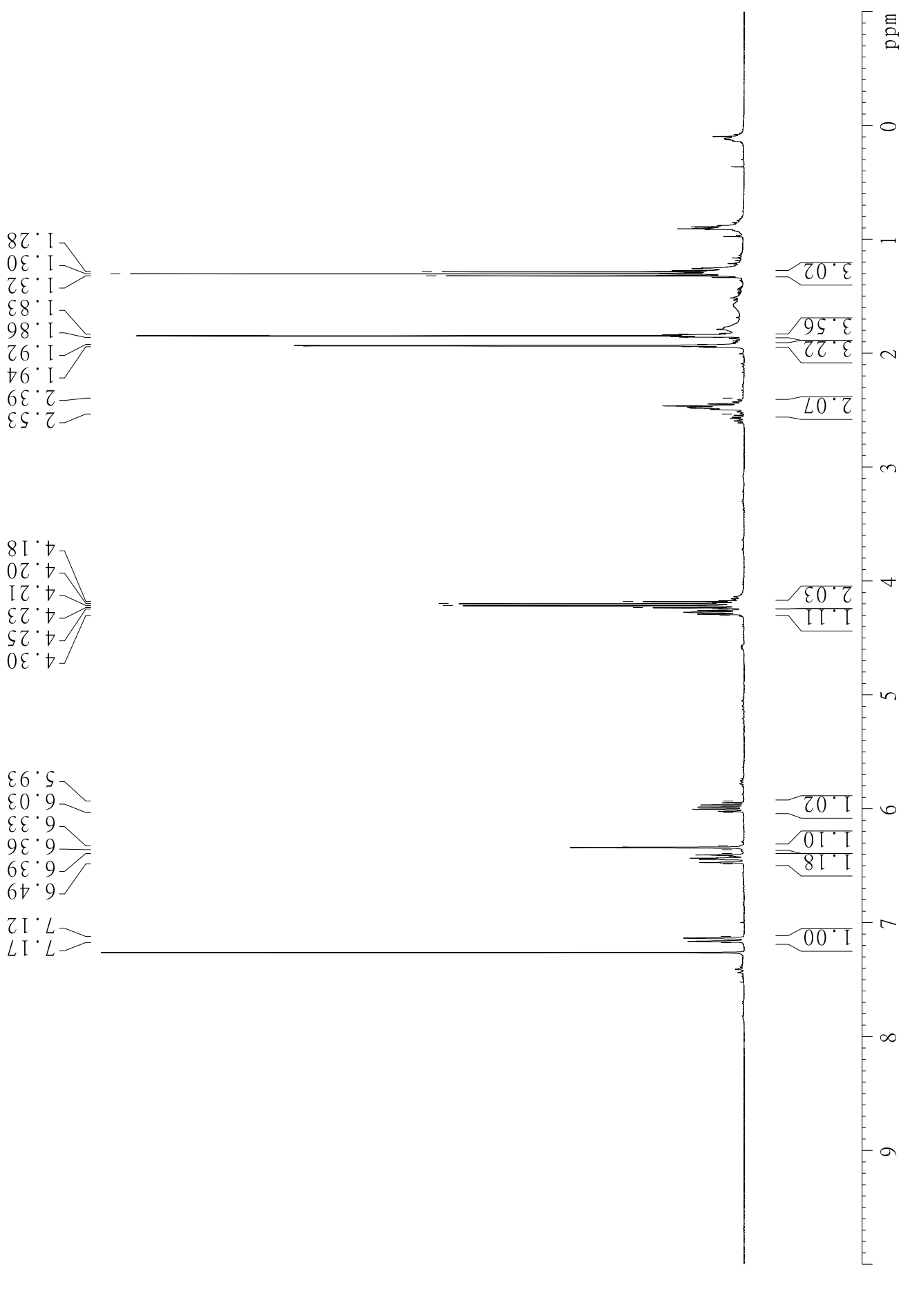

Spectrum 16. ${ }^{1} \mathrm{H}$ spectrum of $\mathbf{5 g}$ measured in $\mathrm{CDCl}_{3}$ at $400 \mathrm{MHz}$. 

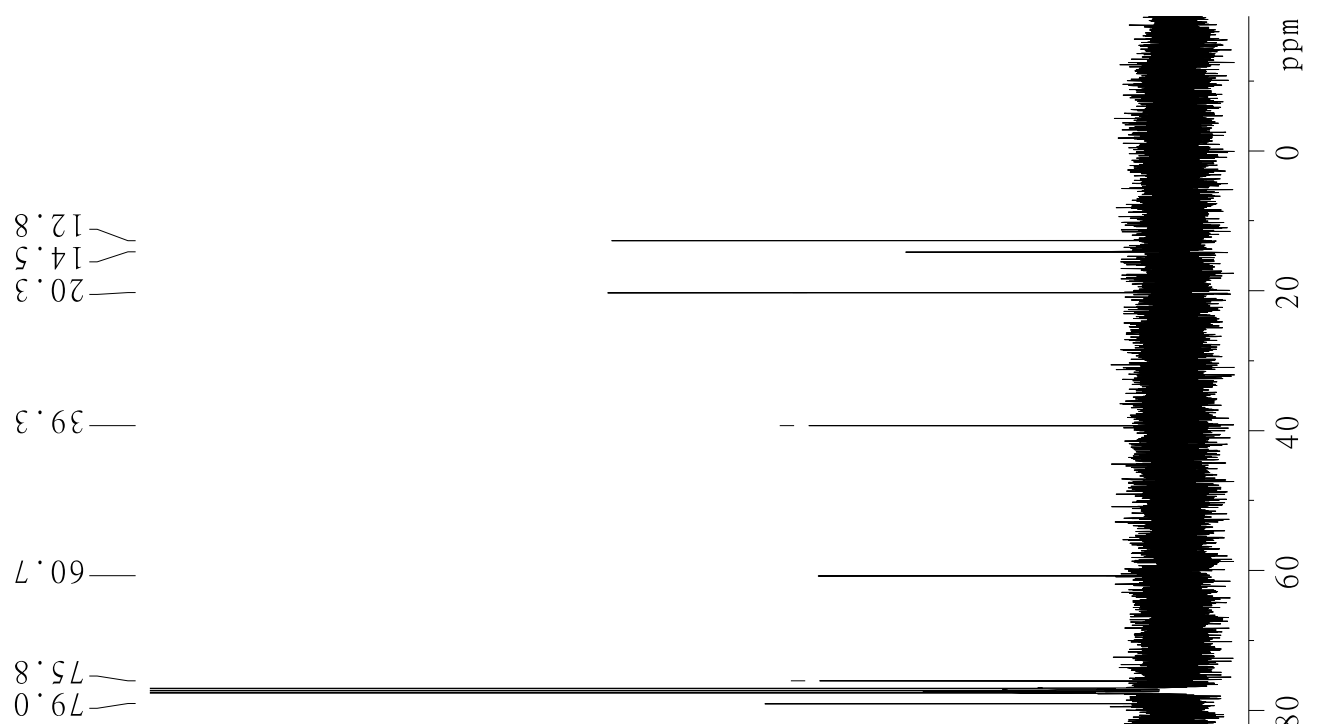

6.97 I -

ह. 62 I

$9^{\circ} 9 \varepsilon \mathrm{I}$

$9^{\circ} L \varepsilon[$

$[\cdot 6 t I$

$9 \cdot 89$ I
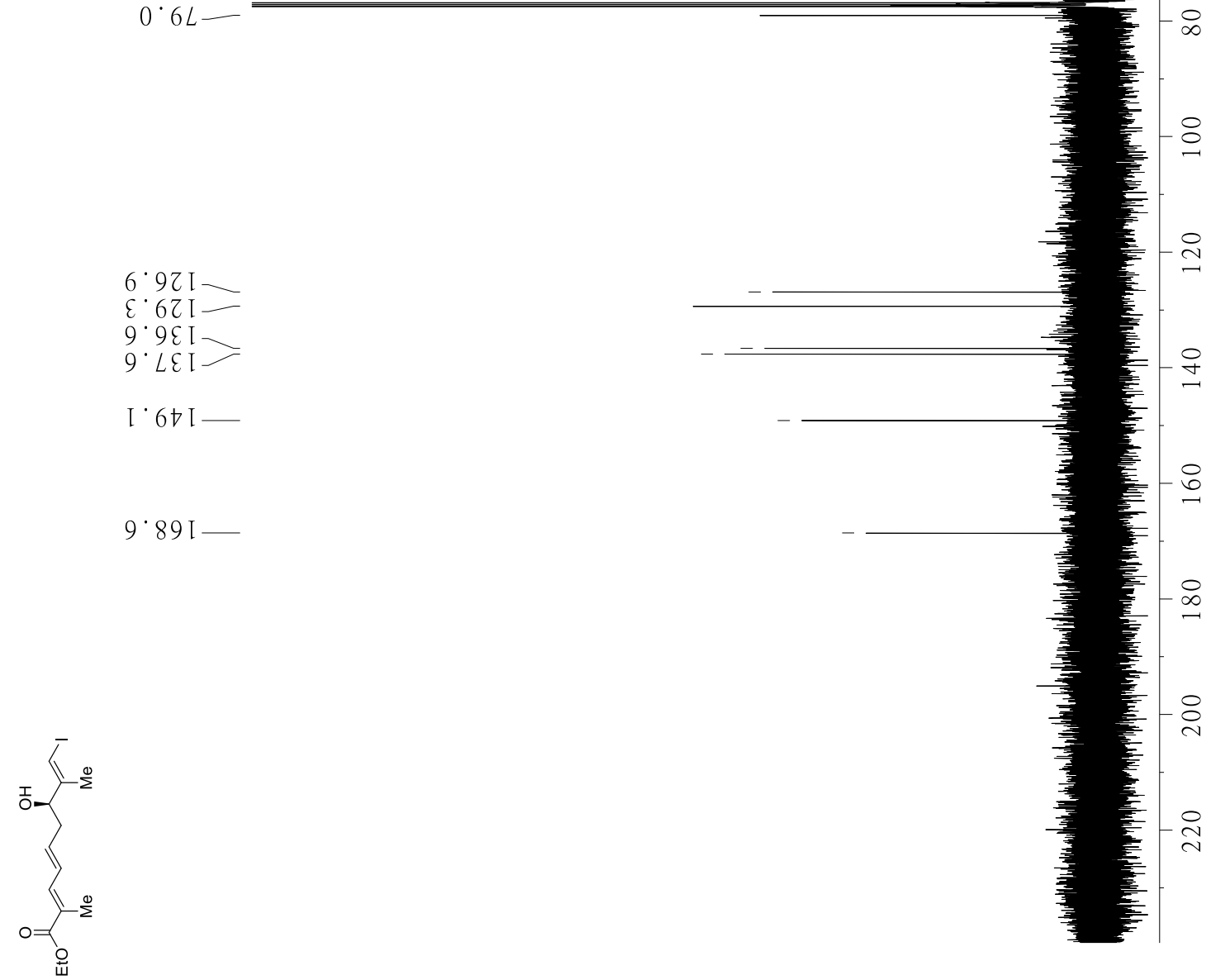

Spectrum 17. ${ }^{13} \mathrm{C}$ spectrum of $\mathbf{5 g}$ measured in $\mathrm{CDCl}_{3}$ at $101 \mathrm{MHz}$. 

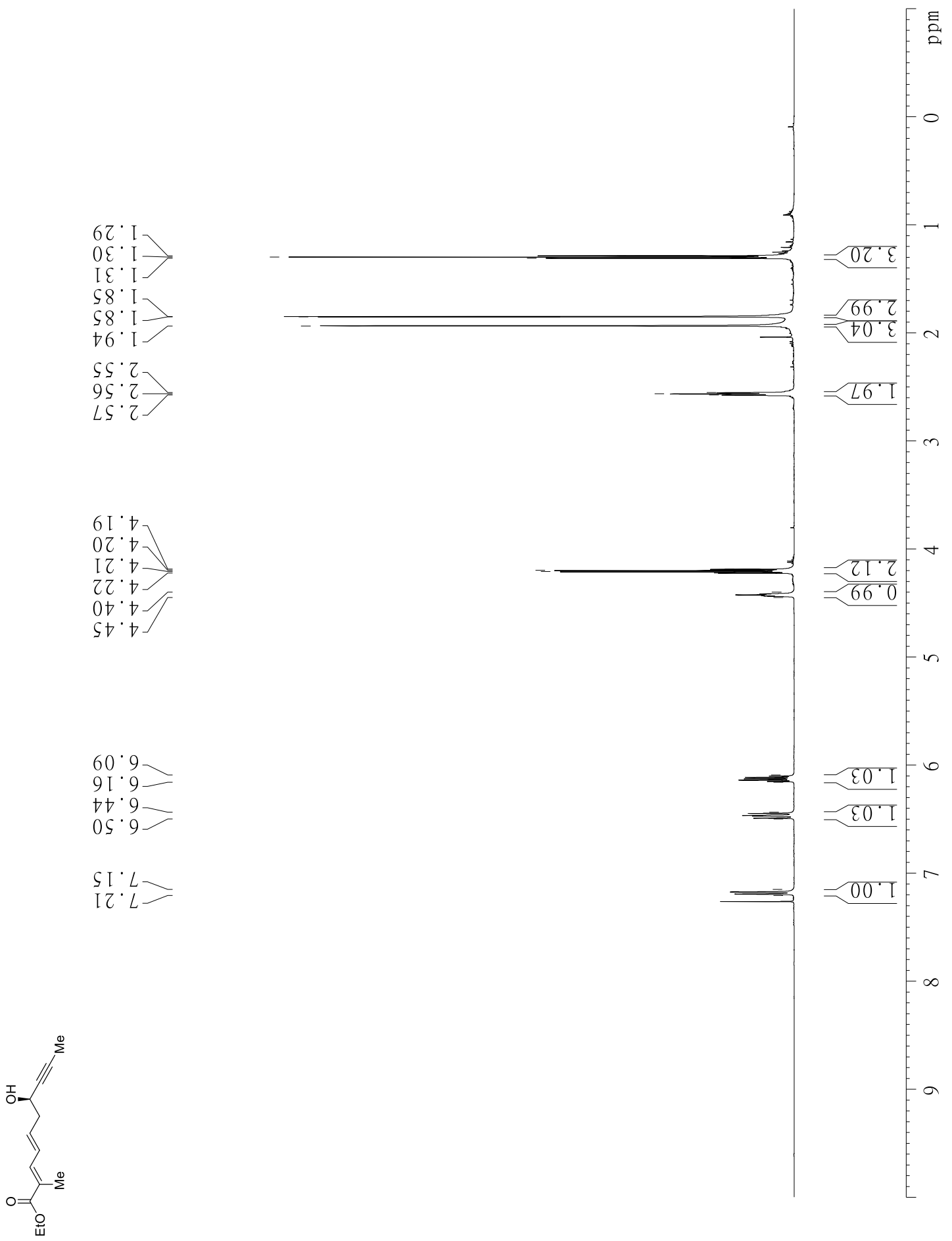

Spectrum 18. ${ }^{1} \mathrm{H}$ spectrum of $\mathbf{5 h}$ measured in $\mathrm{CDCl}_{3}$ at $600 \mathrm{MHz}$. 


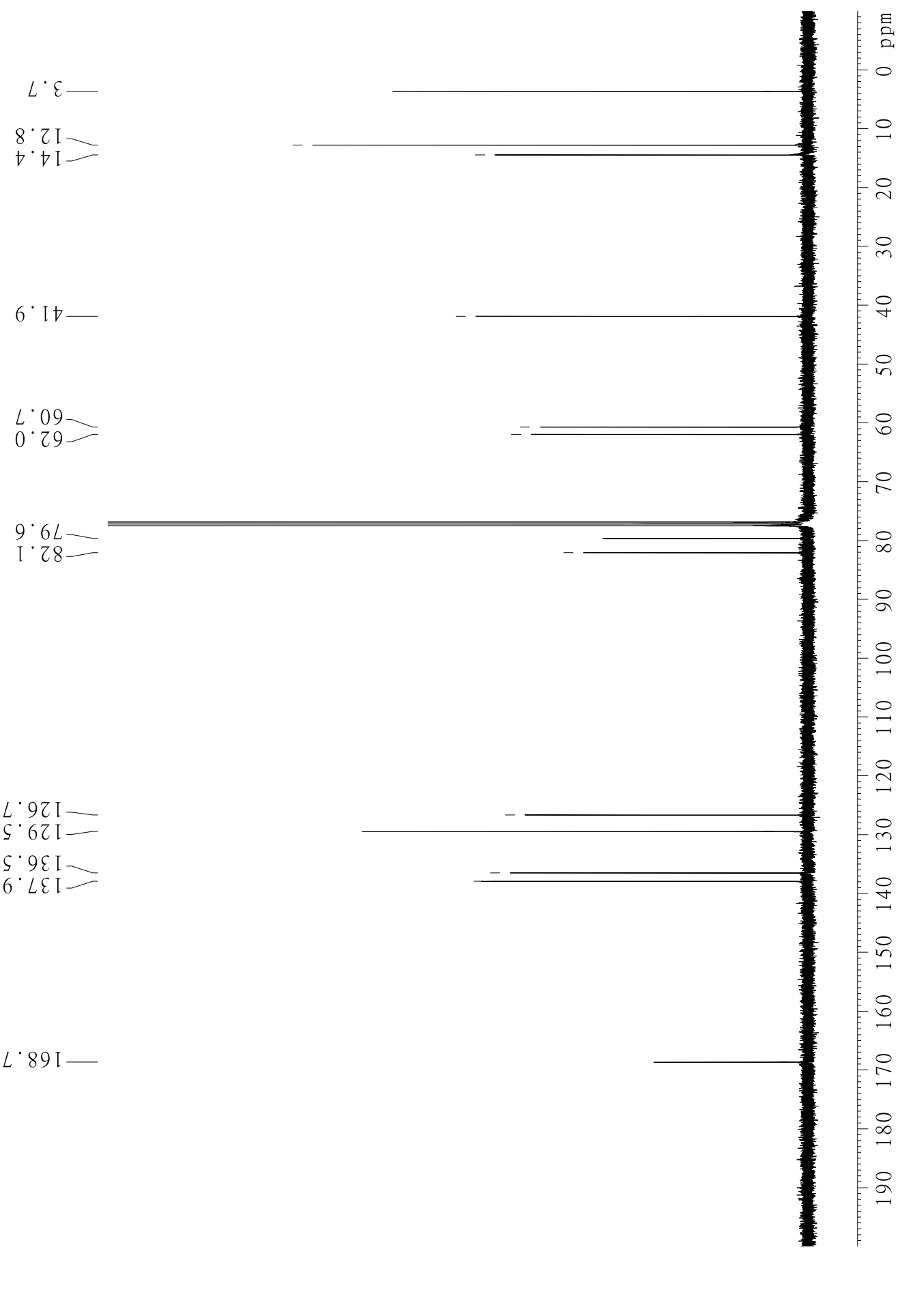

Spectrum 19. ${ }^{13} \mathrm{C}$ spectrum of $\mathbf{5 h}$ measured in $\mathrm{CDCl}_{3}$ at $151 \mathrm{MHz}$. 


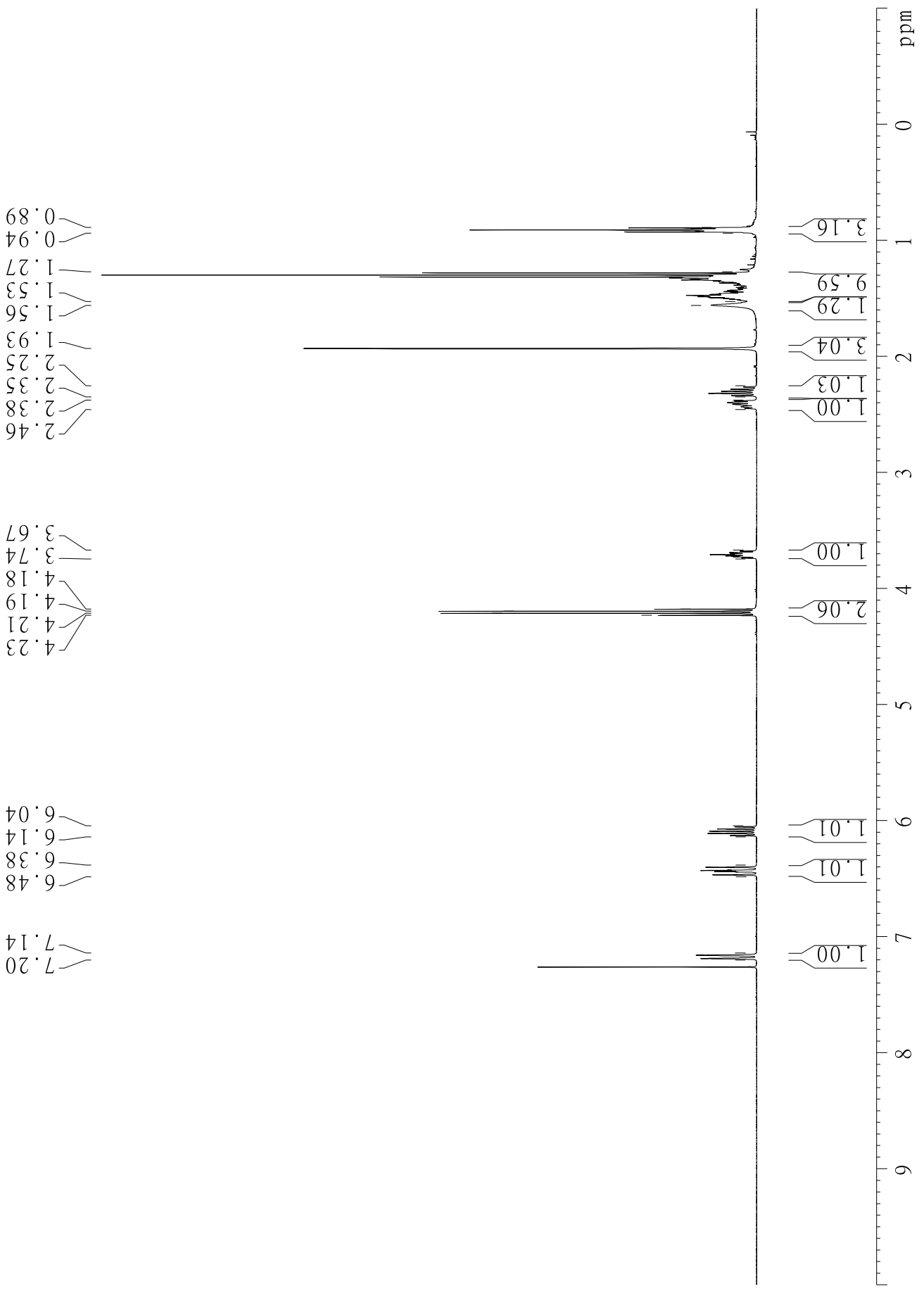

$\sum_{-2}^{\infty}$

Spectrum 20. ${ }^{1} \mathrm{H}$ spectrum of $\mathbf{5 i}$ measured in $\mathrm{CDCl}_{3}$ at $400 \mathrm{MHz}$. 


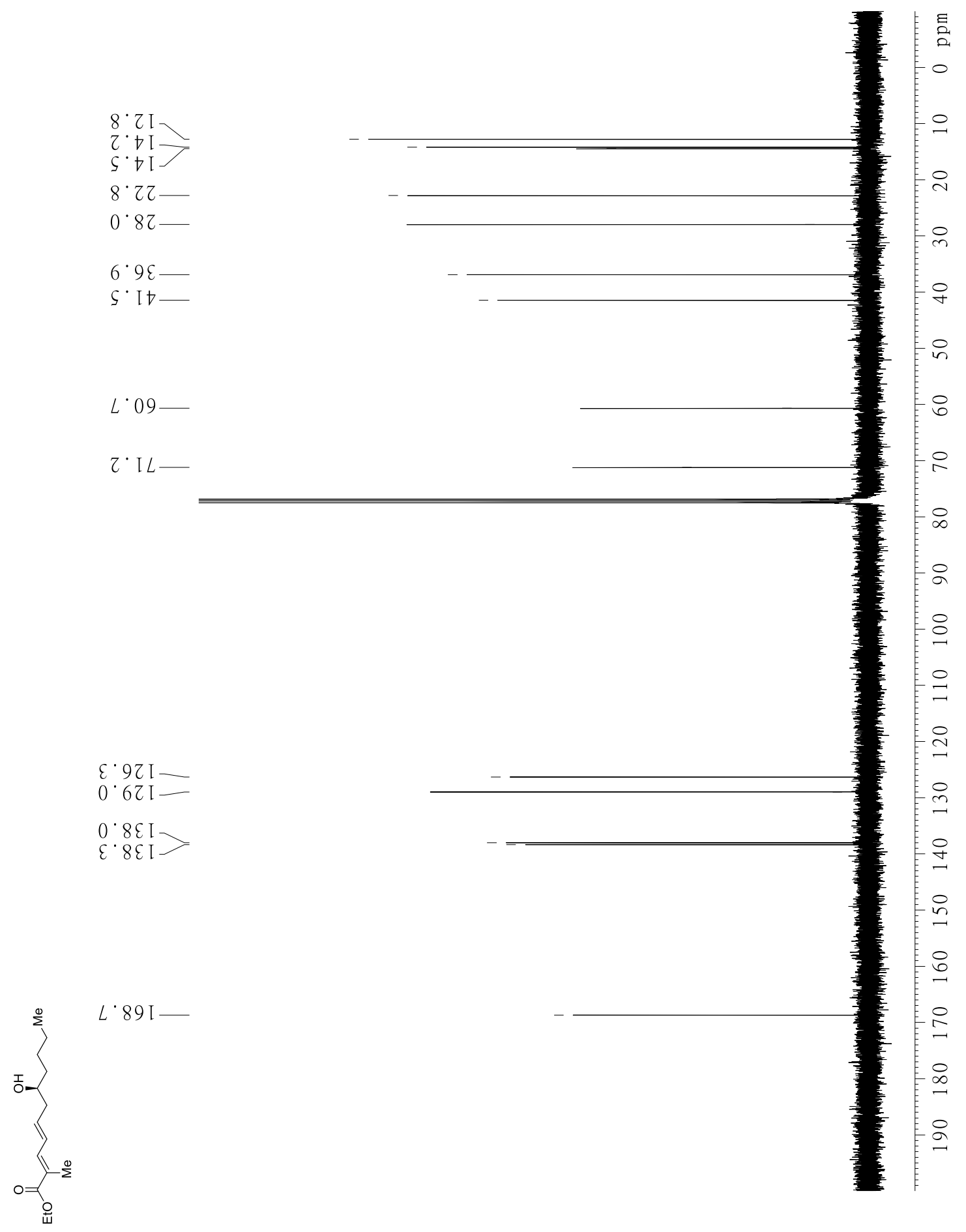

Spectrum 21. ${ }^{13} \mathrm{C}$ spectrum of $5 \mathbf{i}$ measured in $\mathrm{CDCl}_{3}$ at $101 \mathrm{MHz}$. 


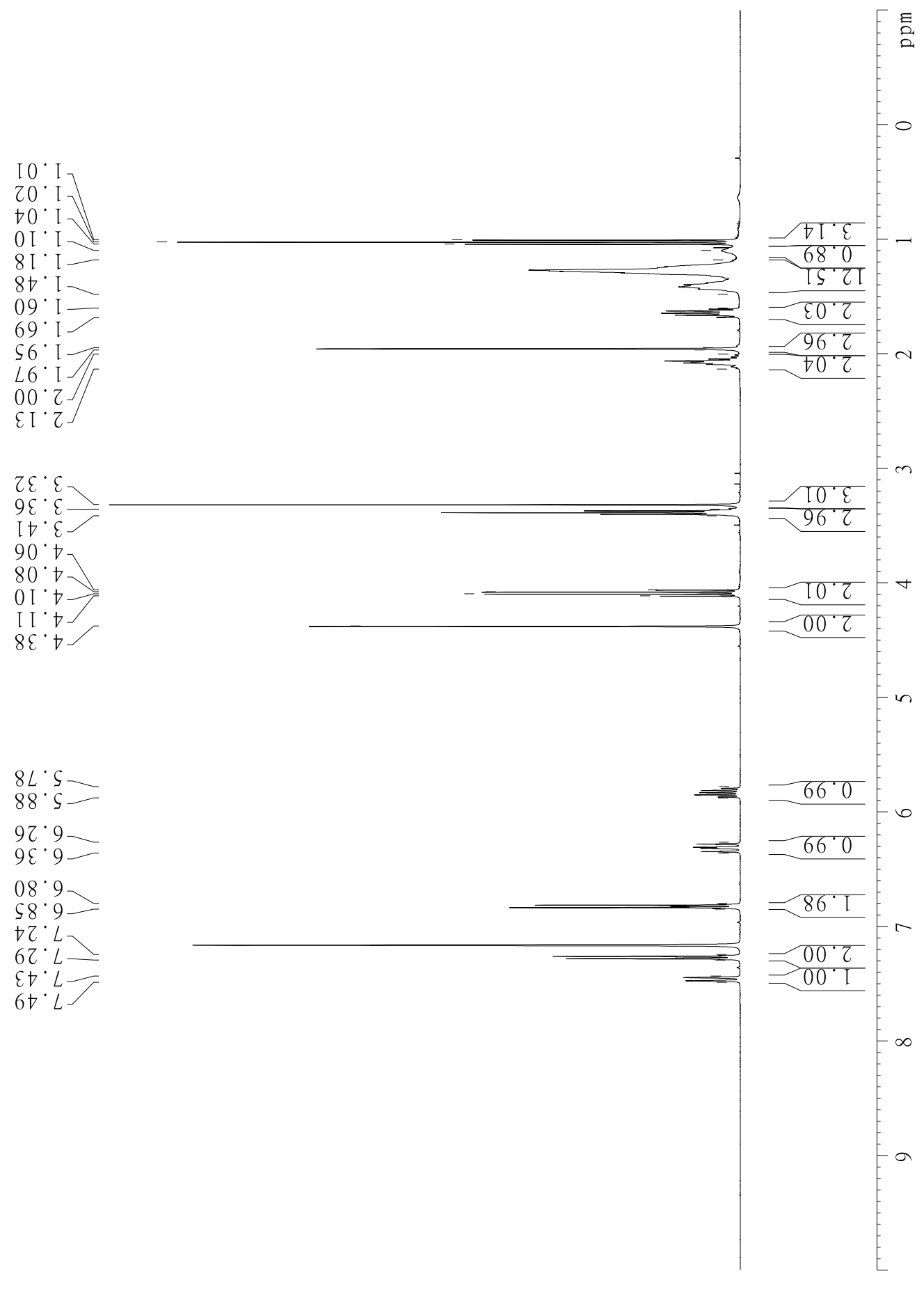

Spectrum 22. ${ }^{1} \mathrm{H}$ spectrum of $\mathbf{5 j}$ measured in $\mathrm{C}_{6} \mathrm{D}_{6}$ at $400 \mathrm{MHz}$. 

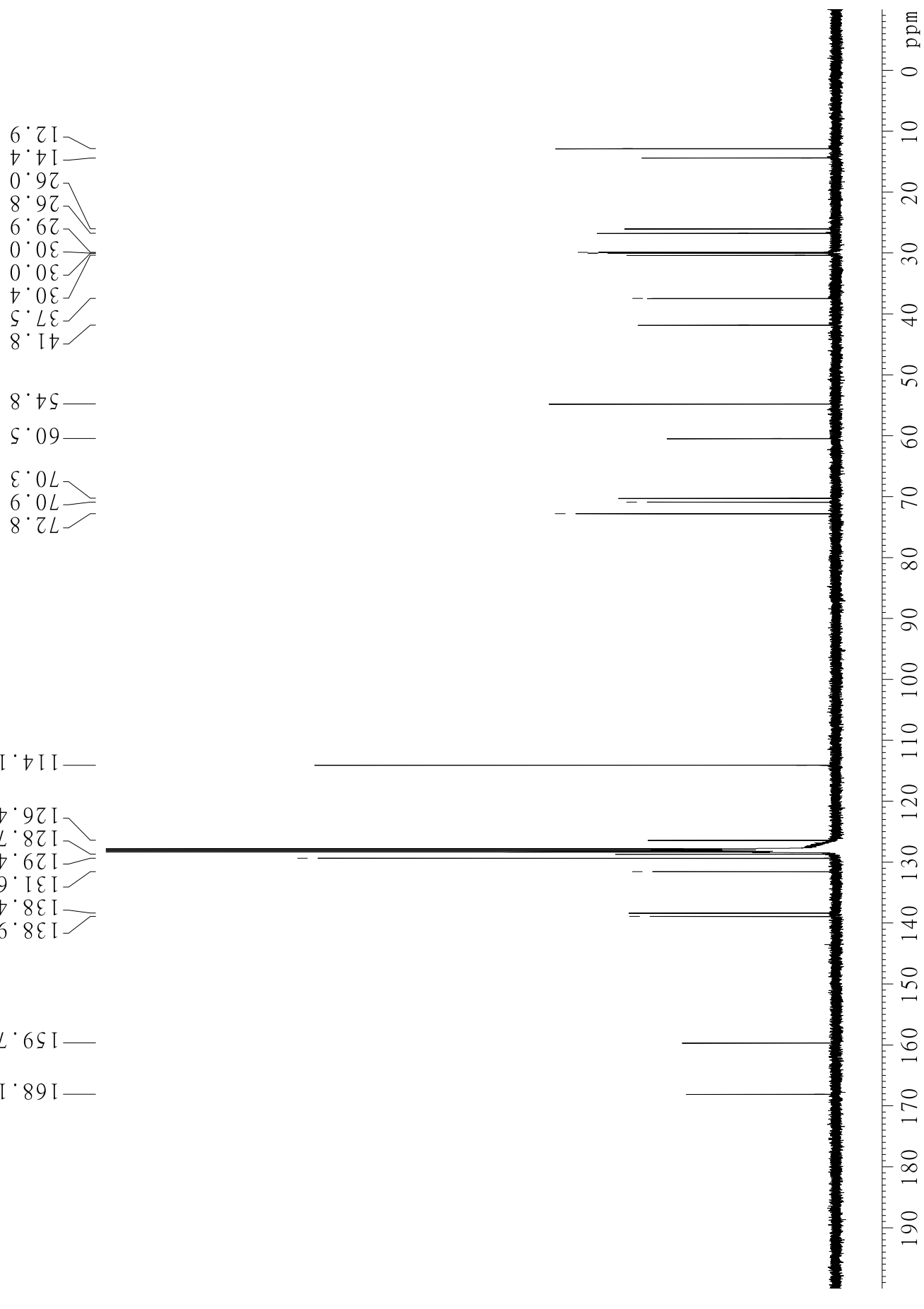

$6^{\circ} 0 L$

$8 \cdot 2 L$

I’ t I I

t.92 I

$L \cdot 8 Z \mathrm{I}$

$t \cdot 62 \mathrm{I}$

$9^{\circ}$ I $\varepsilon$ I

$\nabla^{\circ} 8 \varepsilon \mathrm{I}$

$6 \cdot 8 \varepsilon \mathrm{I}$

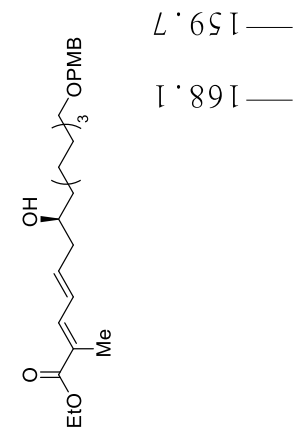

Spectrum 23. ${ }^{13} \mathrm{C}$ spectrum of $\mathbf{5 j}$ measured in $\mathrm{C}_{6} \mathrm{D}_{6}$ at $101 \mathrm{MHz}$. 


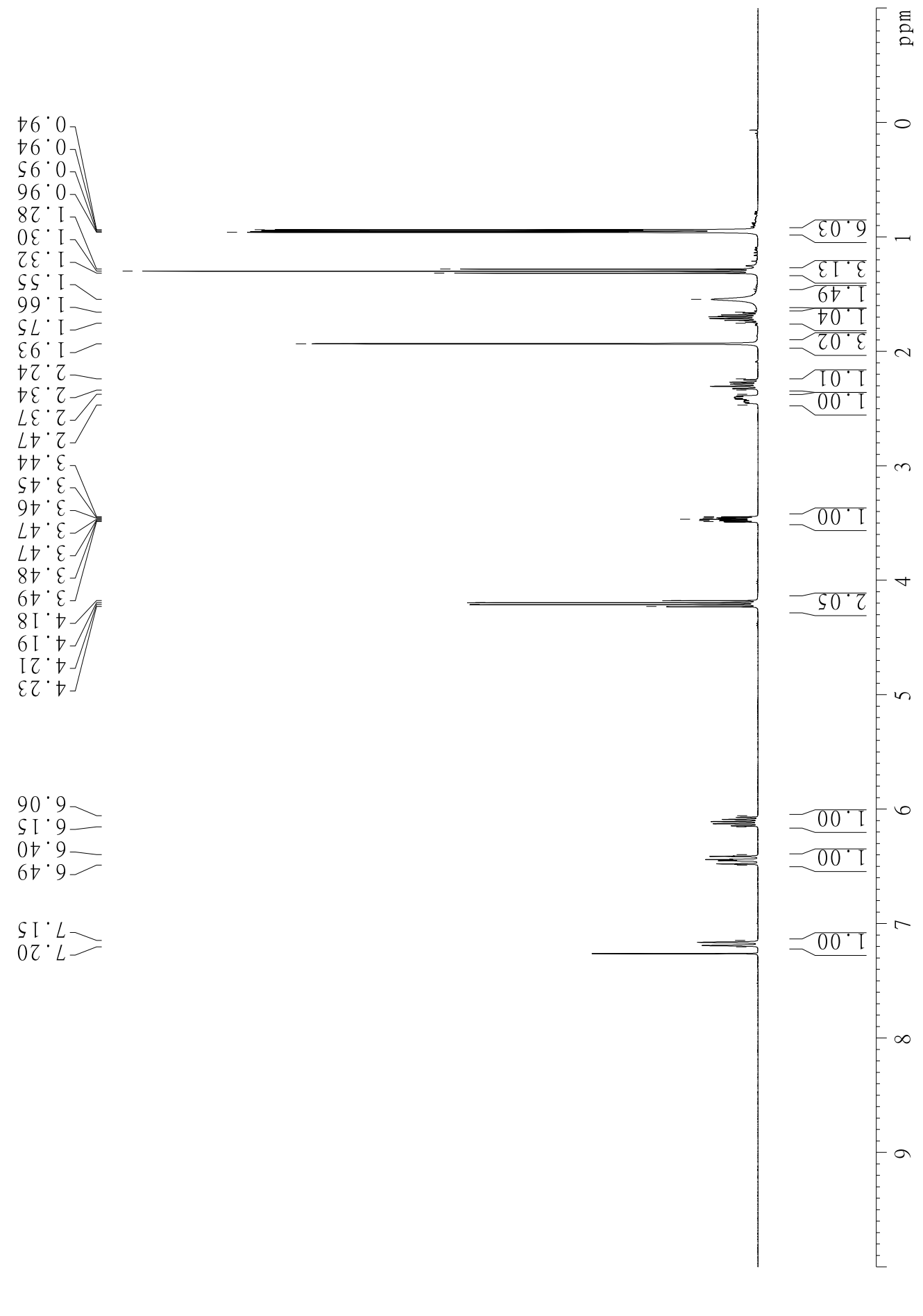

Spectrum 24. ${ }^{1} \mathrm{H}$ spectrum of $\mathbf{5 k}$ measured in $\mathrm{CDCl}_{3}$ at $400 \mathrm{MHz}$. 


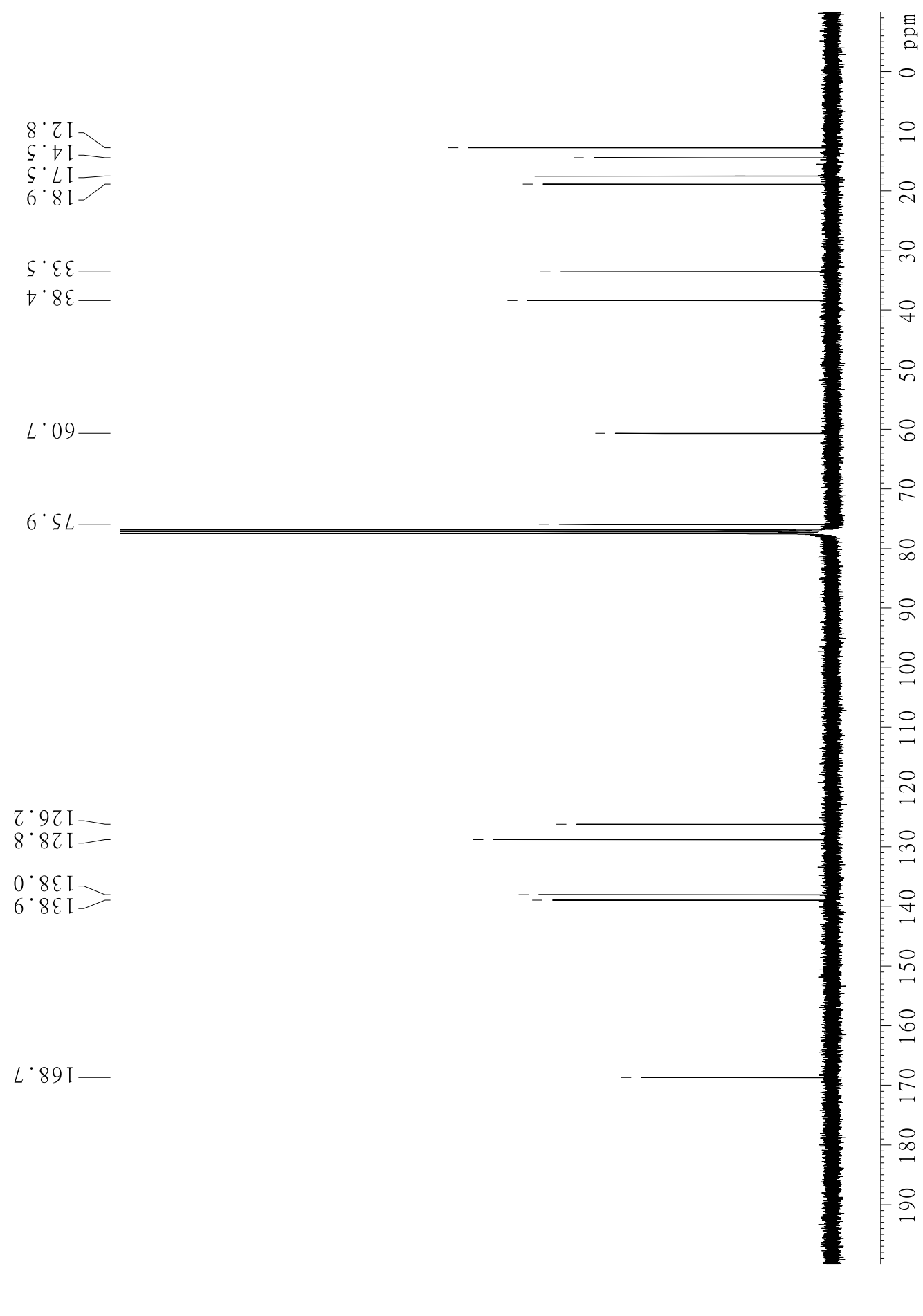

Spectrum $25{ }^{13} \mathrm{C}$ spectrum of $\mathbf{5 k}$ measured in $\mathrm{CDCl}_{3}$ at $101 \mathrm{MHz}$. 


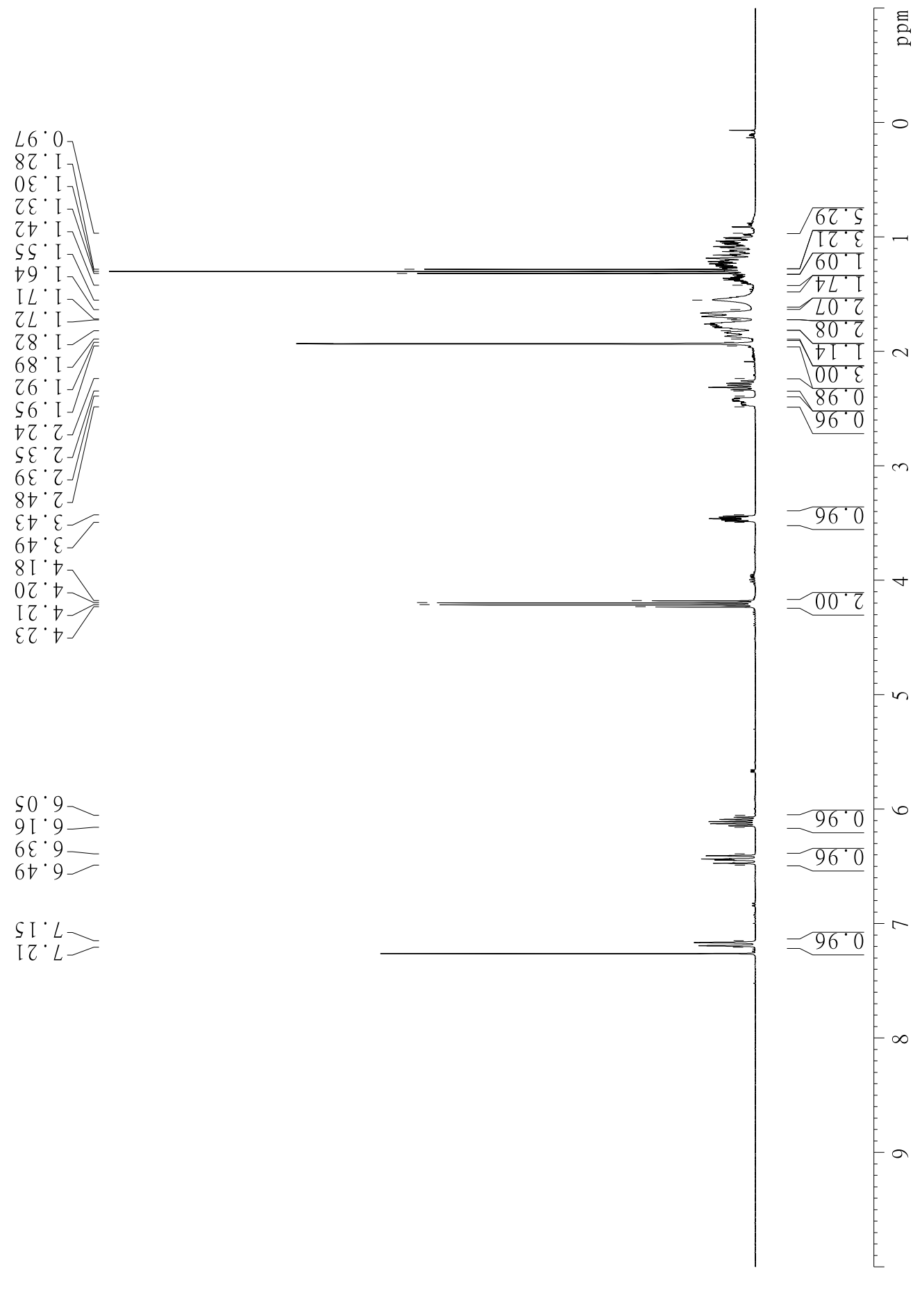

Spectrum 26. ${ }^{1} \mathrm{H}$ spectrum of 51 measured in $\mathrm{CDCl}_{3}$ at $400 \mathrm{MHz}$. 
$8^{\circ} \cdot \mathrm{ZI}-$
$\mathrm{C} \cdot \mathrm{DI}$

r. 9 r

$7 \cdot 97$

$9 \cdot 97$

I. $8 \mathrm{Z}$

$\varepsilon \cdot 62$

$\varepsilon \cdot 8 \varepsilon$

$\downarrow \cdot \varepsilon$ t

$L \cdot 09$

$\varepsilon^{\cdot} \varsigma L$

$7 \cdot 97 \mathrm{I}$

$8 \cdot 8 Z \mathrm{I}-$

$[\cdot 8 \varepsilon \mathrm{I}$

$0 \cdot 6 \varepsilon \mathrm{I}$

L.89I
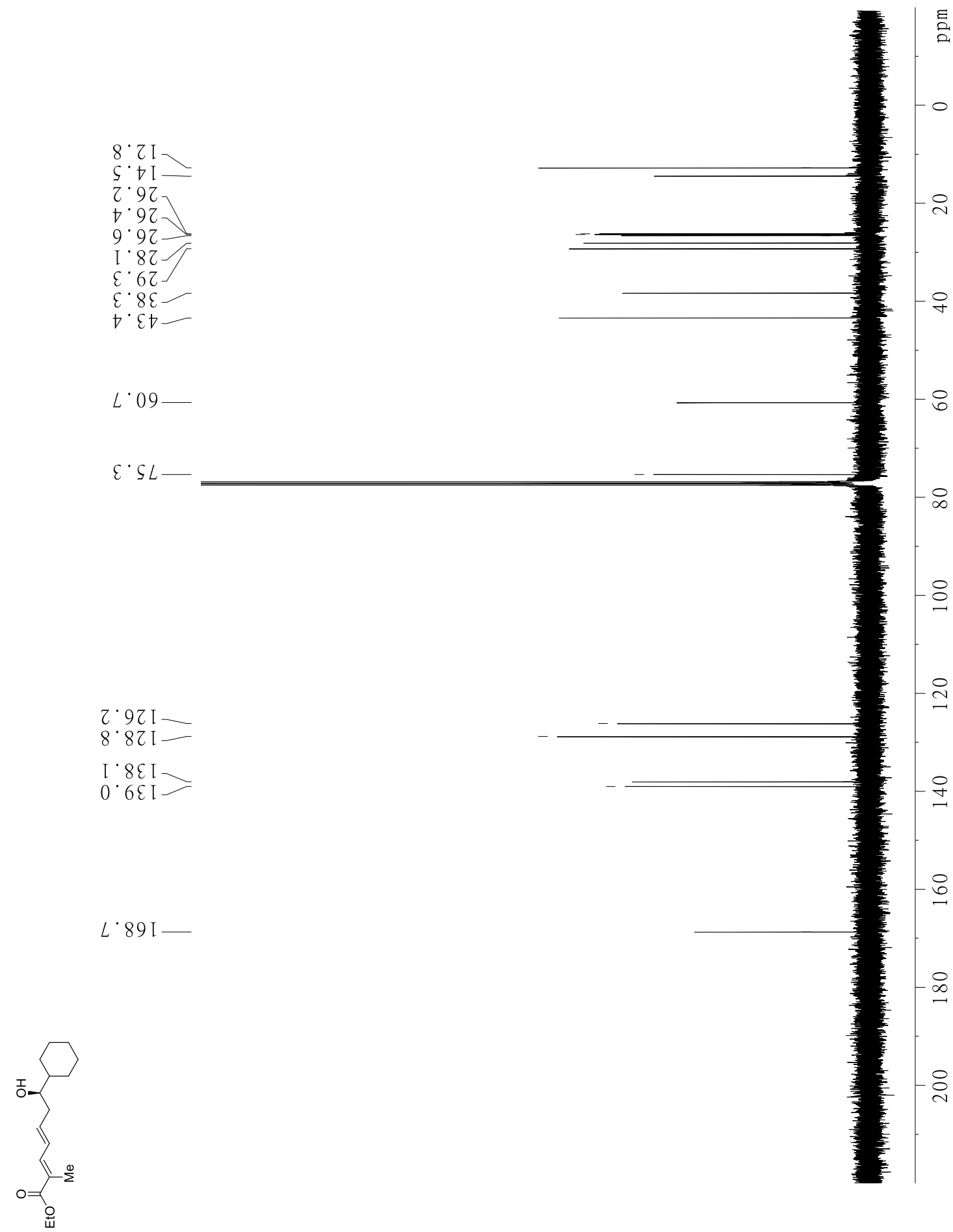

Spectrum 27. ${ }^{13} \mathrm{C}$ spectrum of $\mathbf{5 l}$ measured in $\mathrm{CDCl}_{3}$ at $101 \mathrm{MHz}$. 


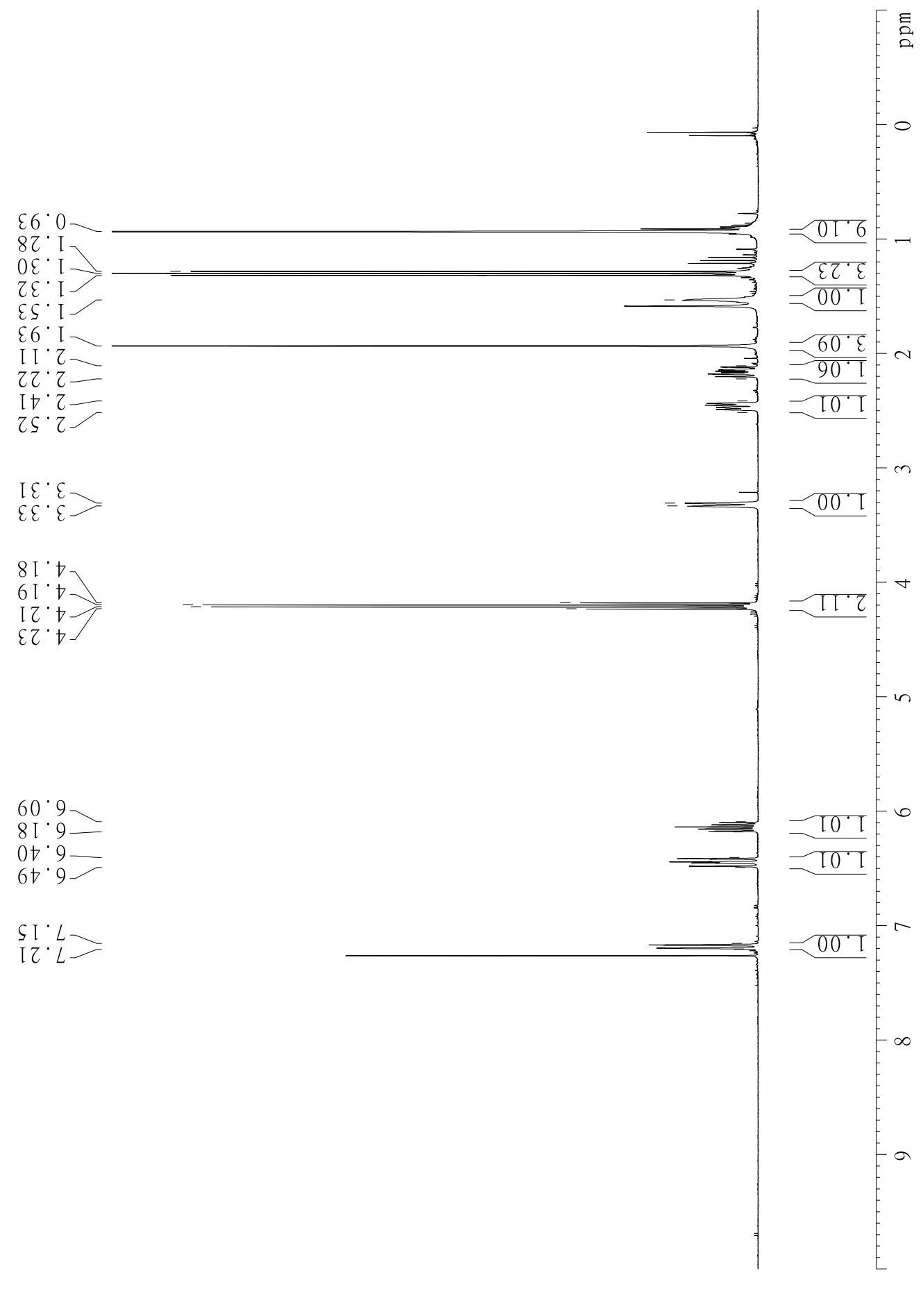

Spectrum 28. ${ }^{1} \mathrm{H}$ spectrum of $\mathbf{5 m}$ measured in $\mathrm{CDCl}_{3}$ at $400 \mathrm{MHz}$. 


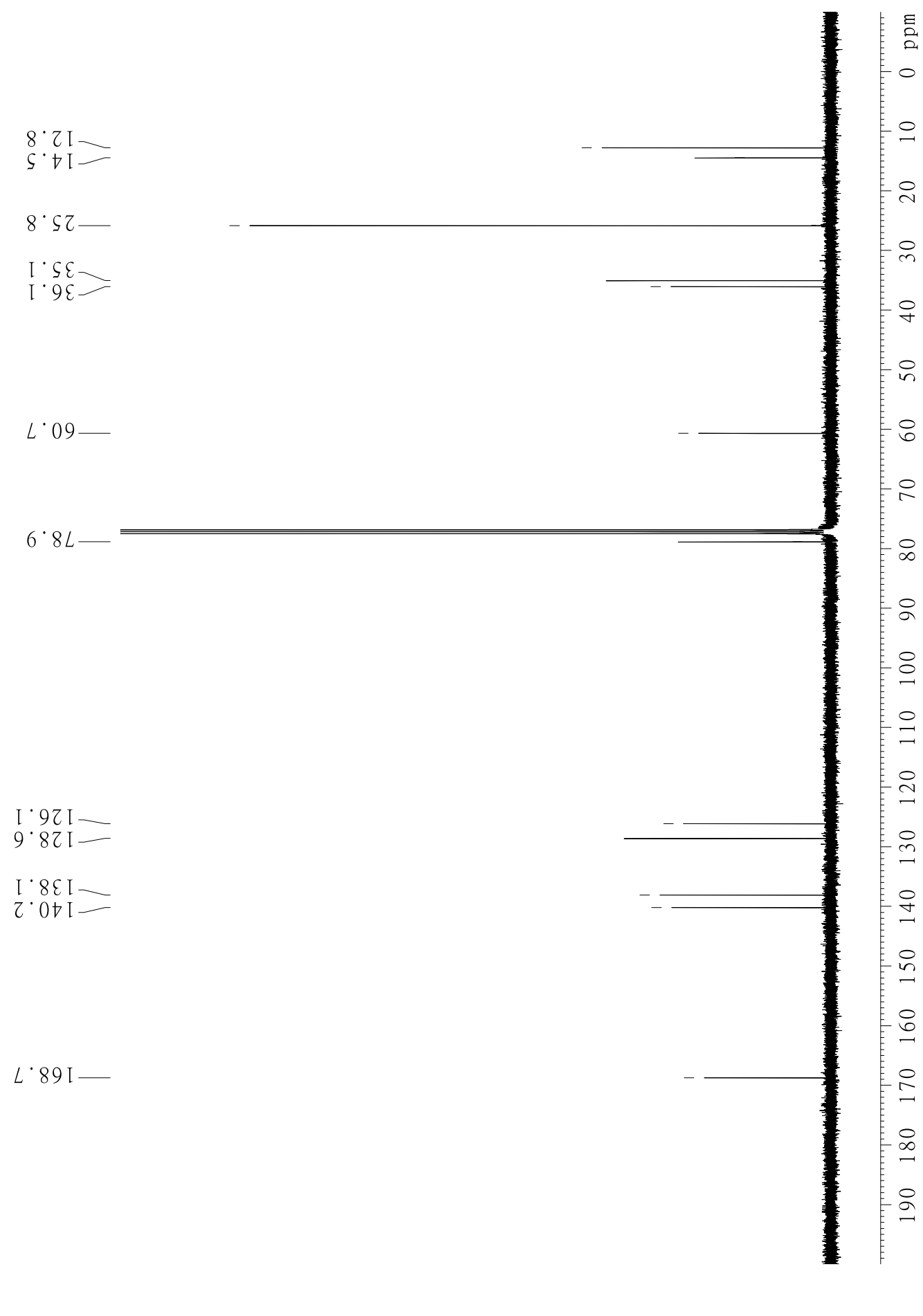

Spectrum 29. ${ }^{13} \mathrm{C}$ spectrum of $\mathbf{5 m}$ measured in $\mathrm{CDCl}_{3}$ at $101 \mathrm{MHz}$. 


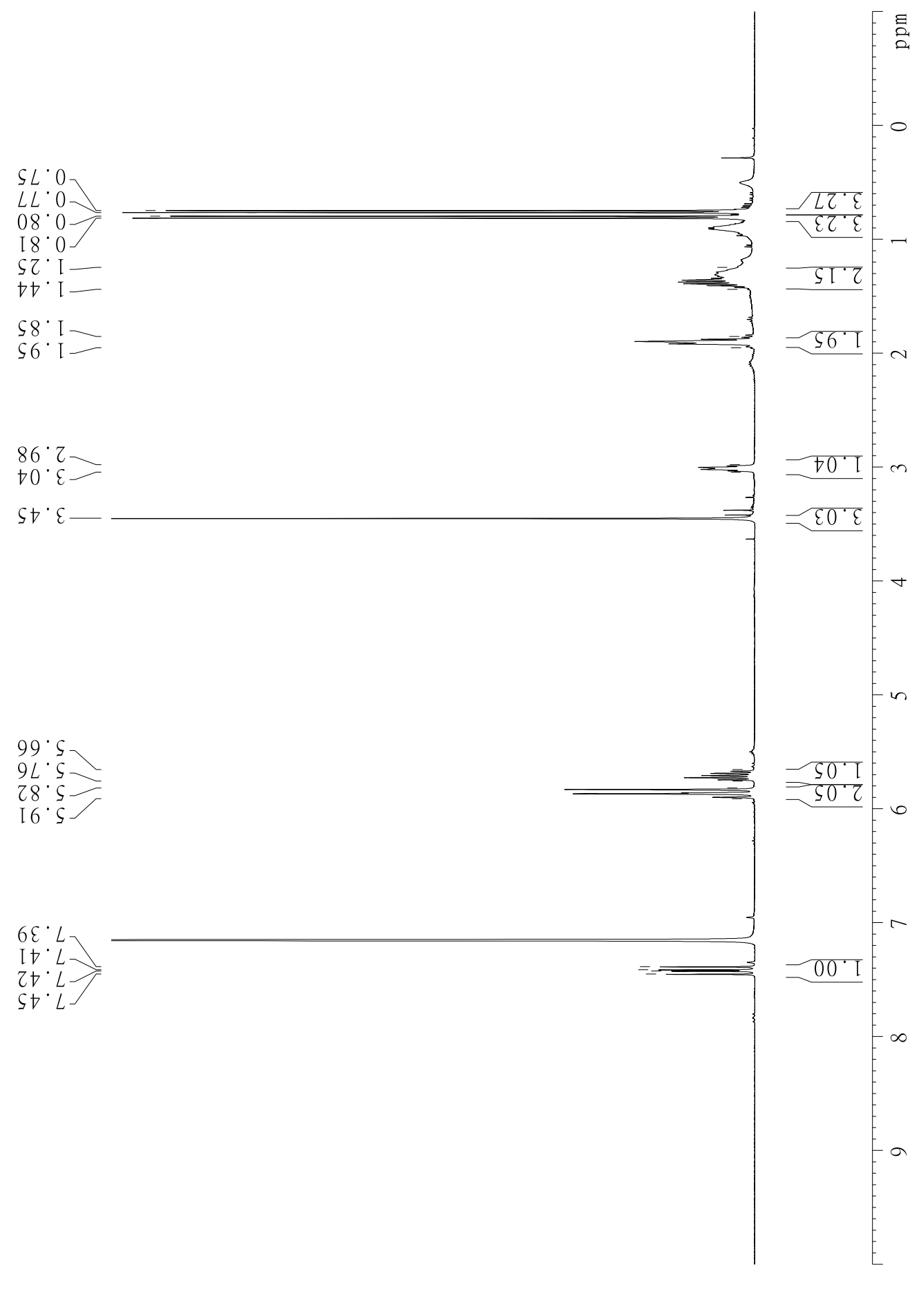

Spectrum 30. ${ }^{1} \mathrm{H}$ spectrum of $5 \mathrm{n}$ measured in $\mathrm{C}_{6} \mathrm{D}_{6}$ at $400 \mathrm{MHz}$. 


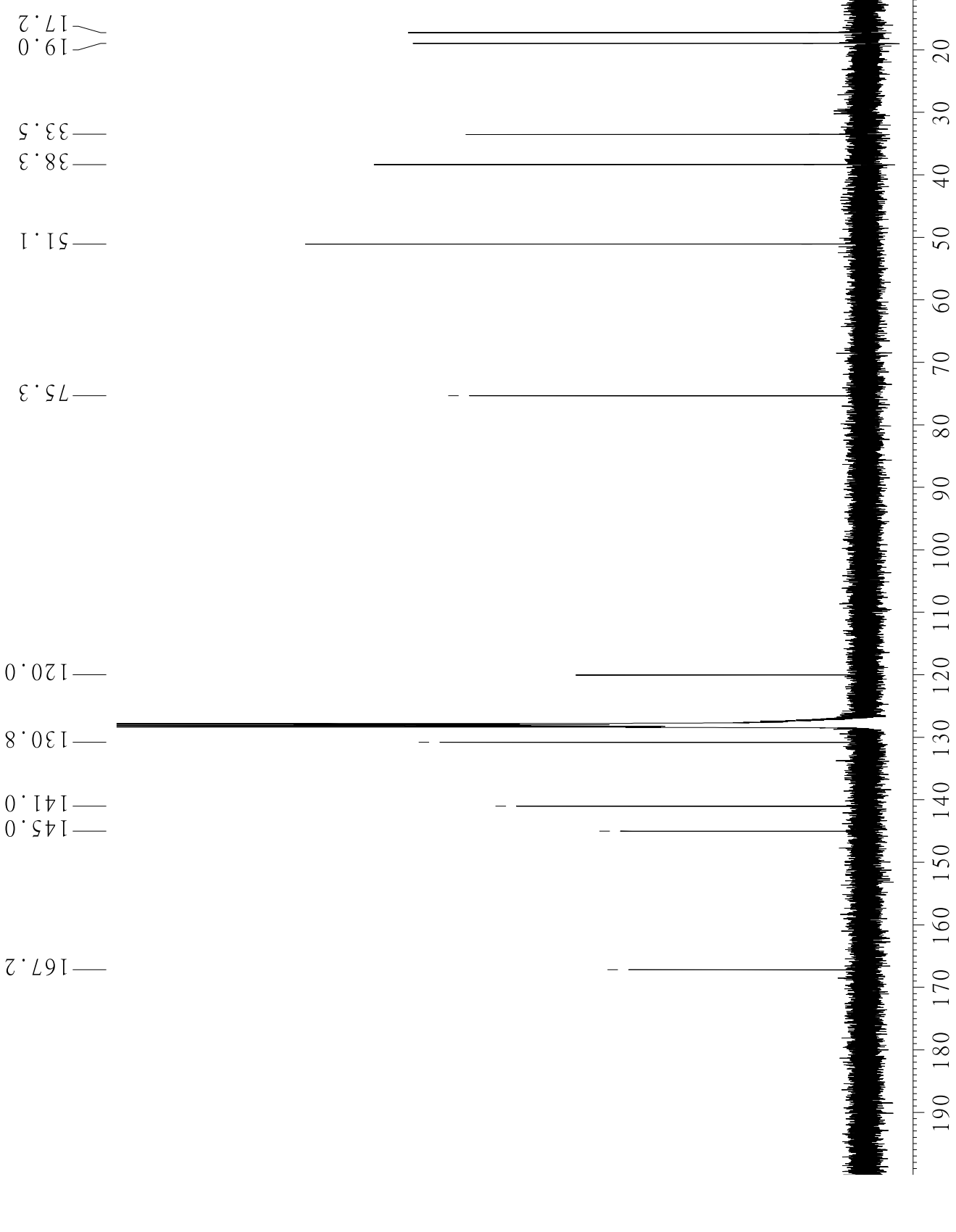

Spectrum 31. ${ }^{13} \mathrm{C}$ spectrum of $\mathbf{5 n}$ measured in $\mathrm{C}_{6} \mathrm{D}_{6}$ at $101 \mathrm{MHz}$. 

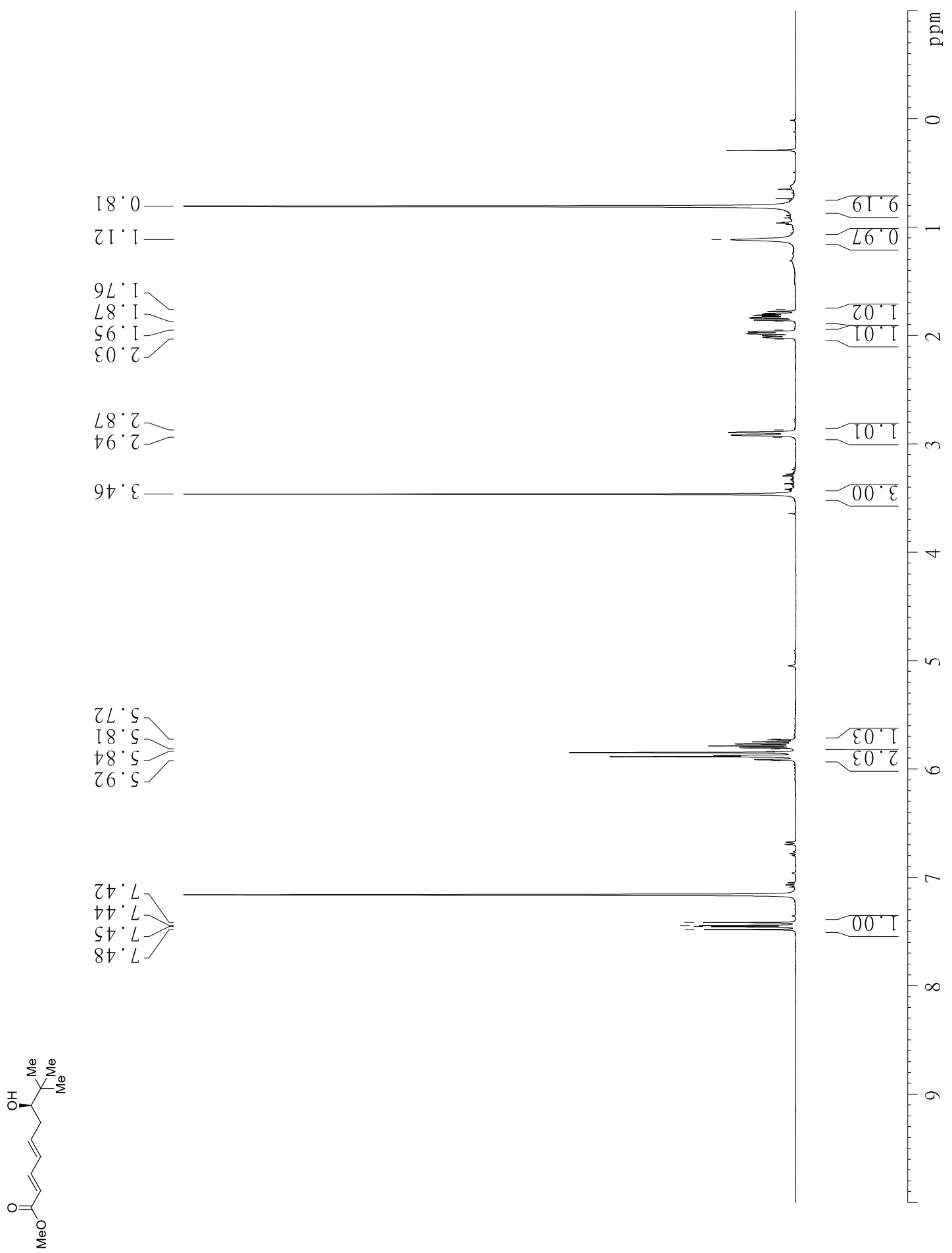

Spectrum 32. ${ }^{1} \mathrm{H}$ spectrum of 50 measured in $\mathrm{C}_{6} \mathrm{D}_{6}$ at $400 \mathrm{MHz}$. 


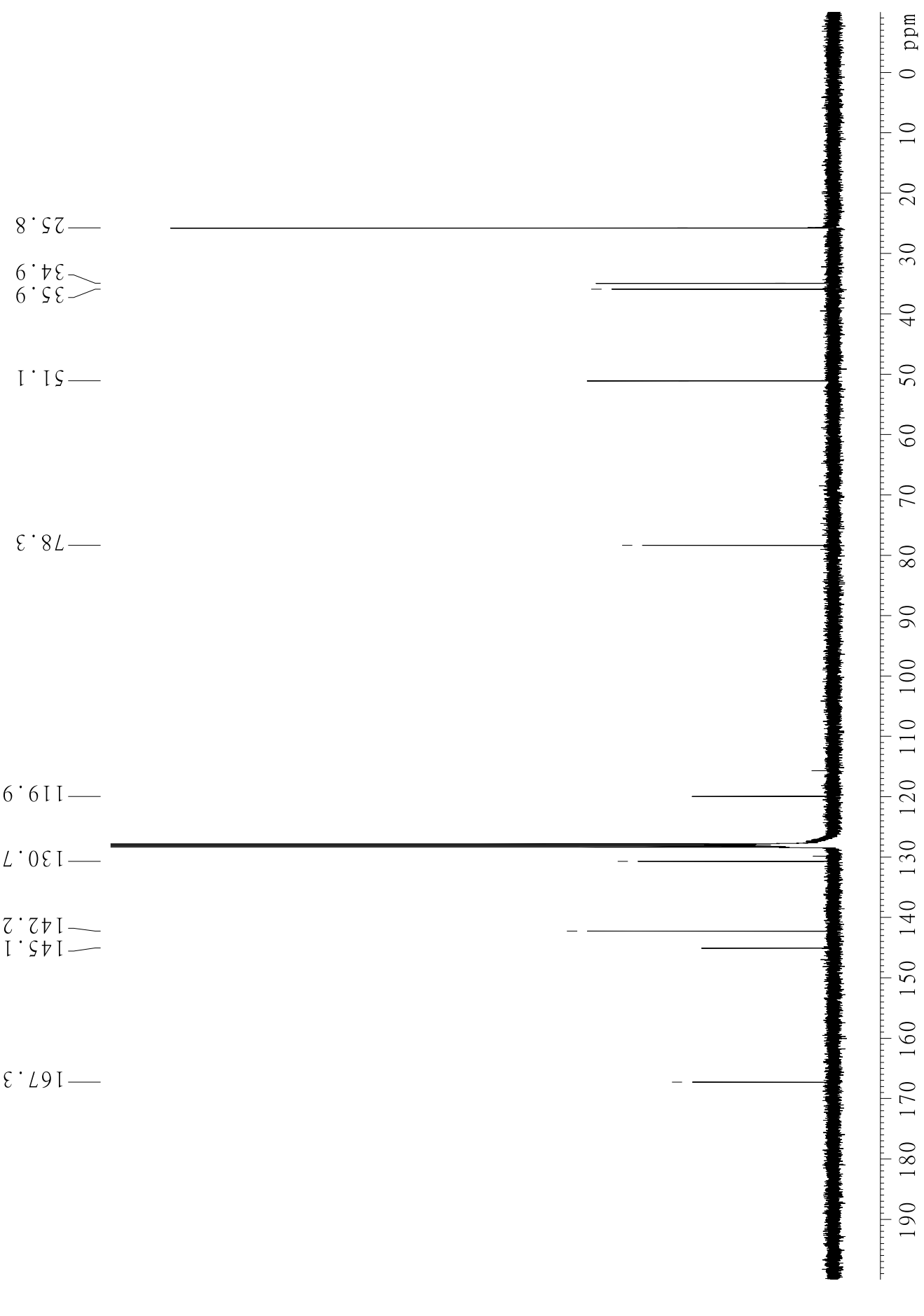

Spectrum 33. ${ }^{13} \mathrm{C}$ spectrum of $\mathbf{5 o}$ measured in $\mathrm{C}_{6} \mathrm{D}_{6}$ at $101 \mathrm{MHz}$. 


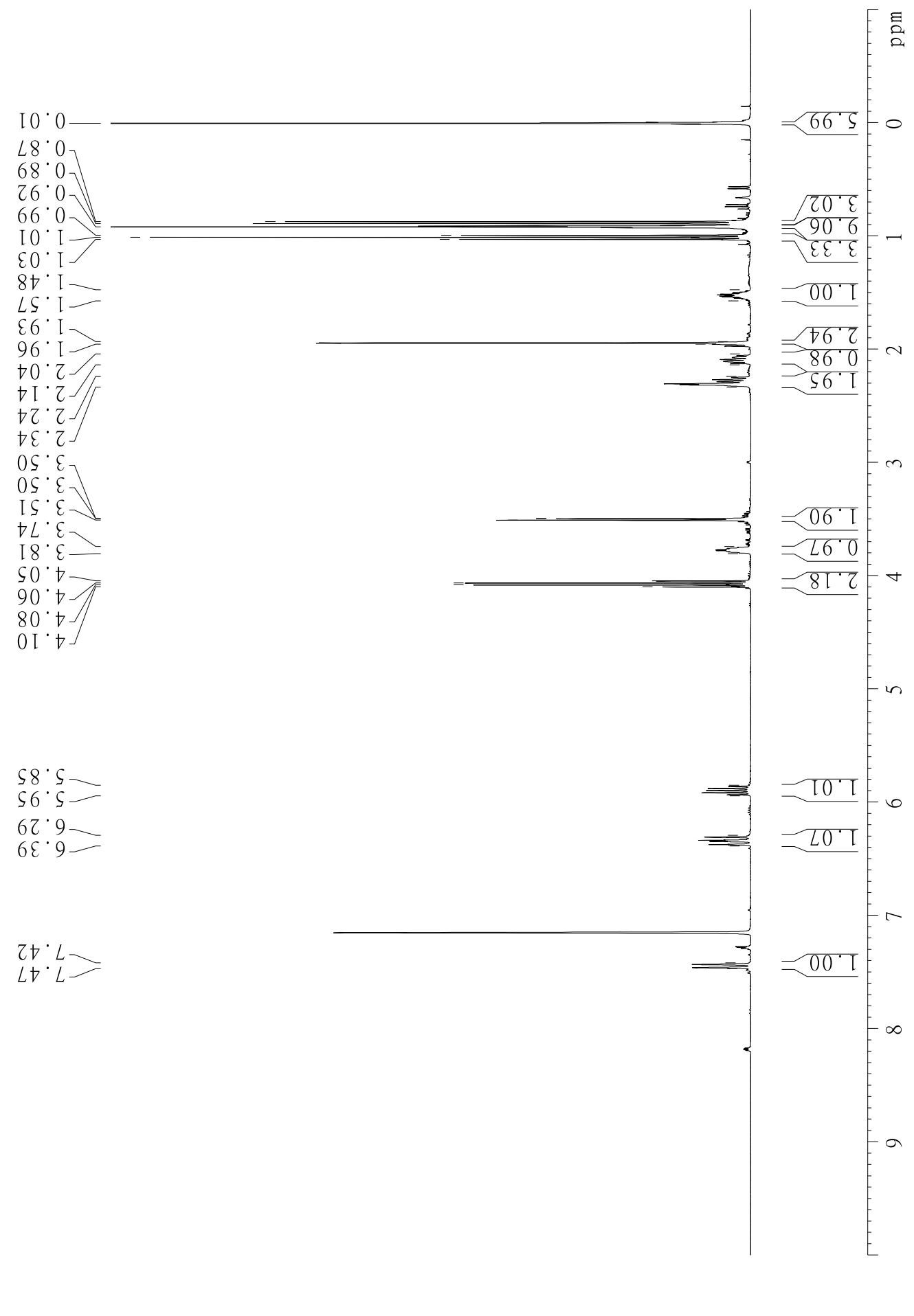

Spectrum 34. ${ }^{1} \mathrm{H}$ spectrum of $5 p$ measured in $\mathrm{C}_{6} \mathrm{D}_{6}$ at $400 \mathrm{MHz}$. 


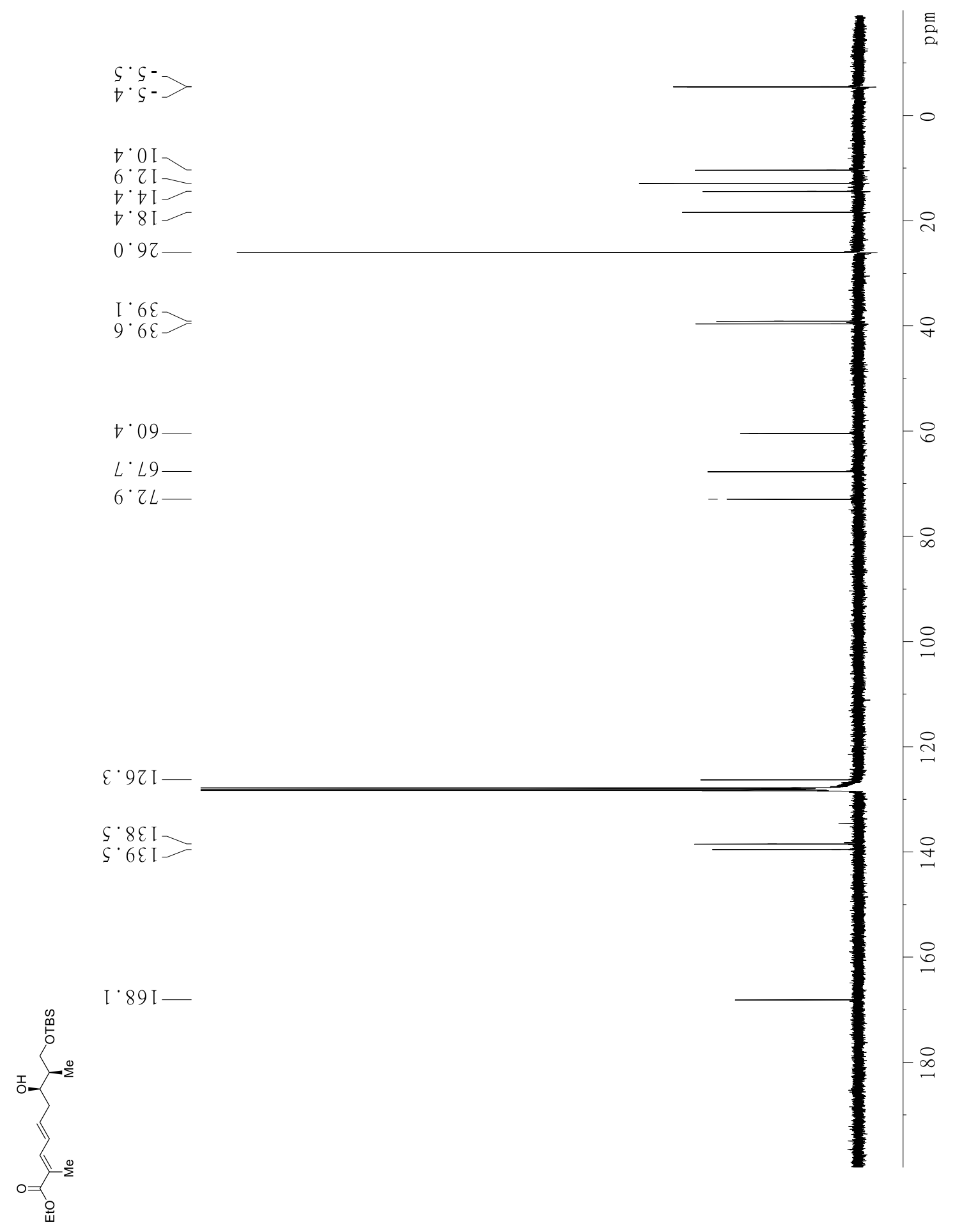

Spectrum 35. ${ }^{13} \mathrm{C}$ spectrum of $\mathbf{5 p}$ measured in $\mathrm{C}_{6} \mathrm{D}_{6}$ at $101 \mathrm{MHz}$. 


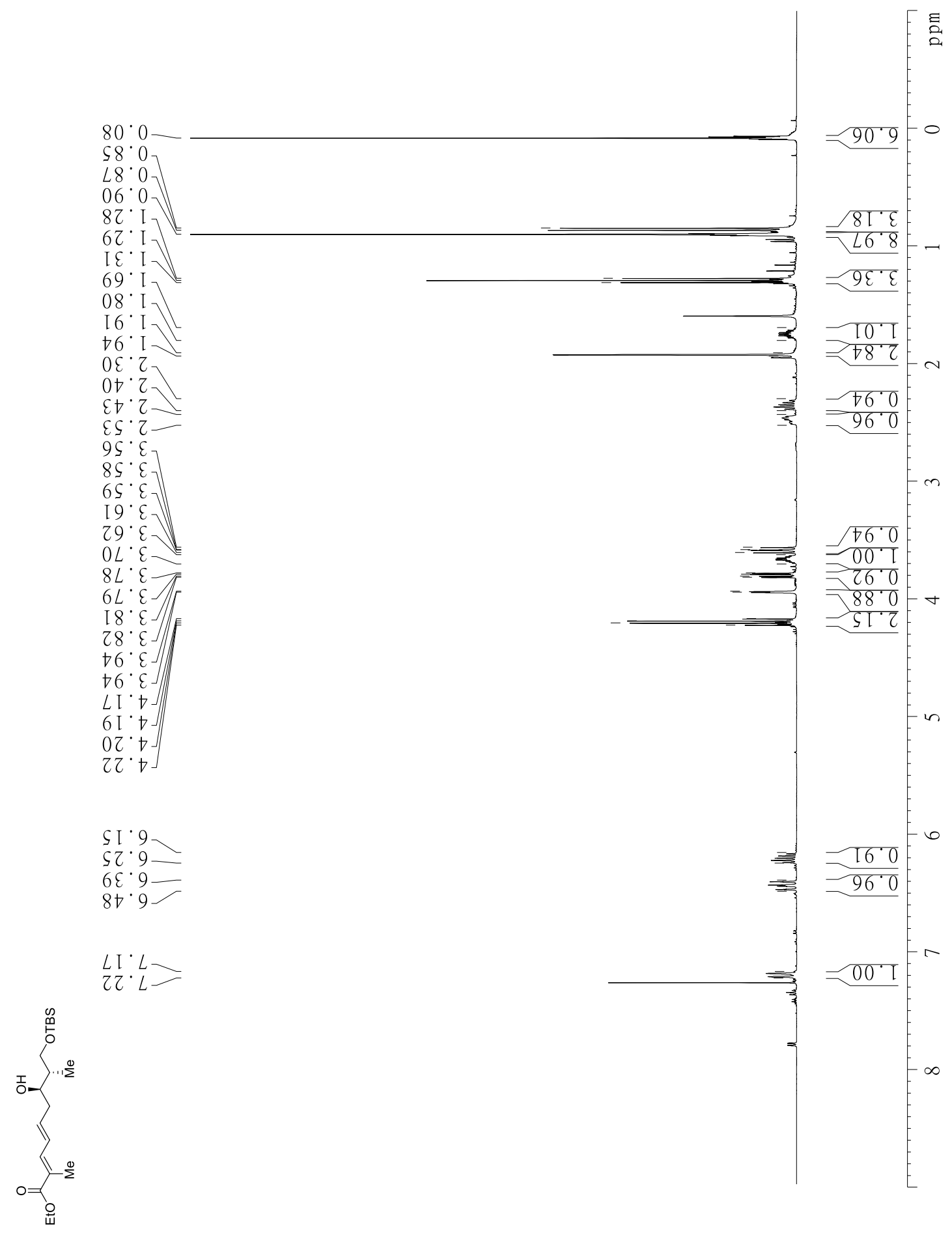

Spectrum 36. ${ }^{1} \mathrm{H}$ spectrum of $\mathbf{5 q}$ measured in $\mathrm{CDCl}_{3}$ at $600 \mathrm{MHz}$. 


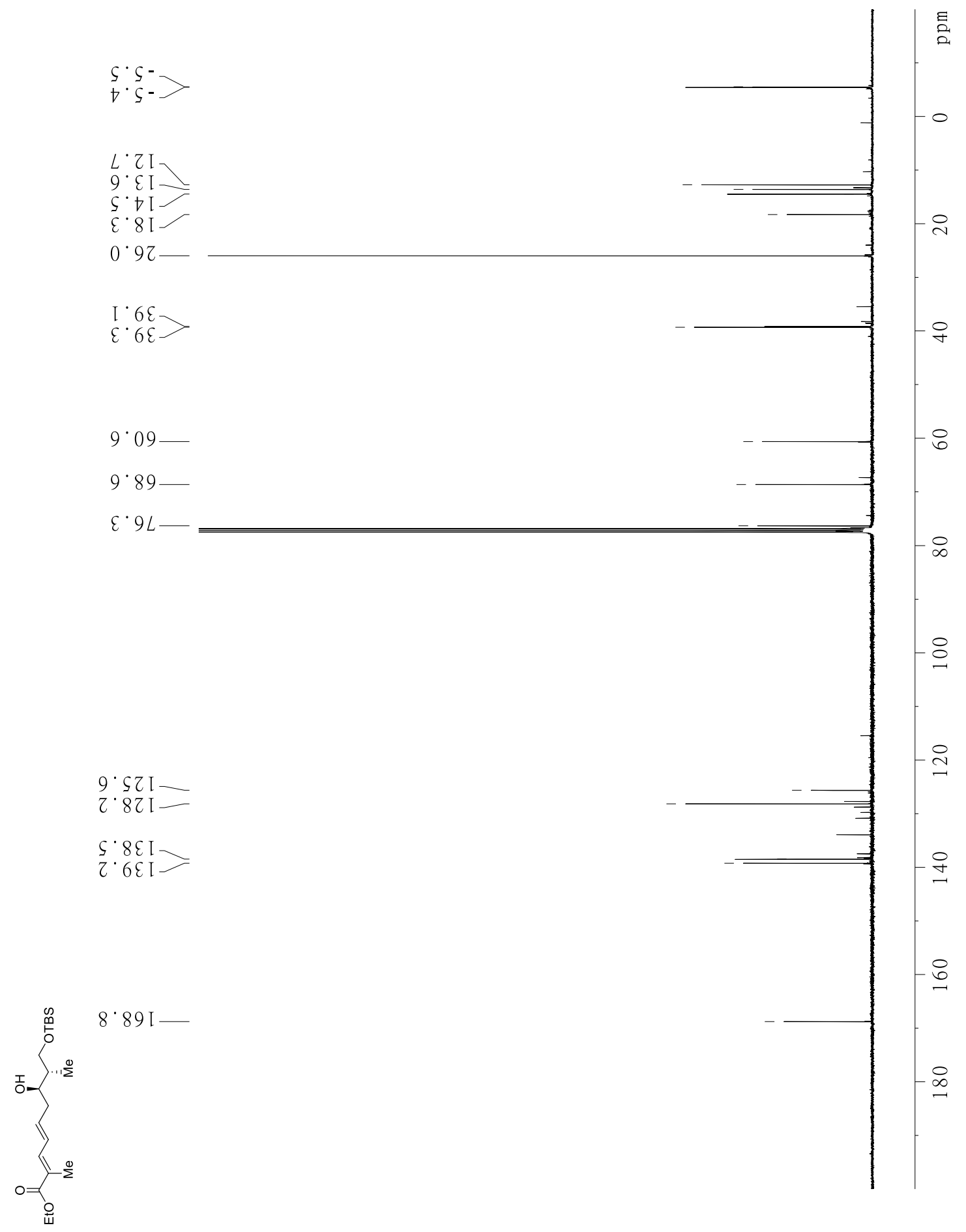

Spectrum 37. ${ }^{13} \mathrm{C}$ spectrum of $\mathbf{5 q}$ measured in $\mathrm{CDCl}_{3}$ at $151 \mathrm{MHz}$. 


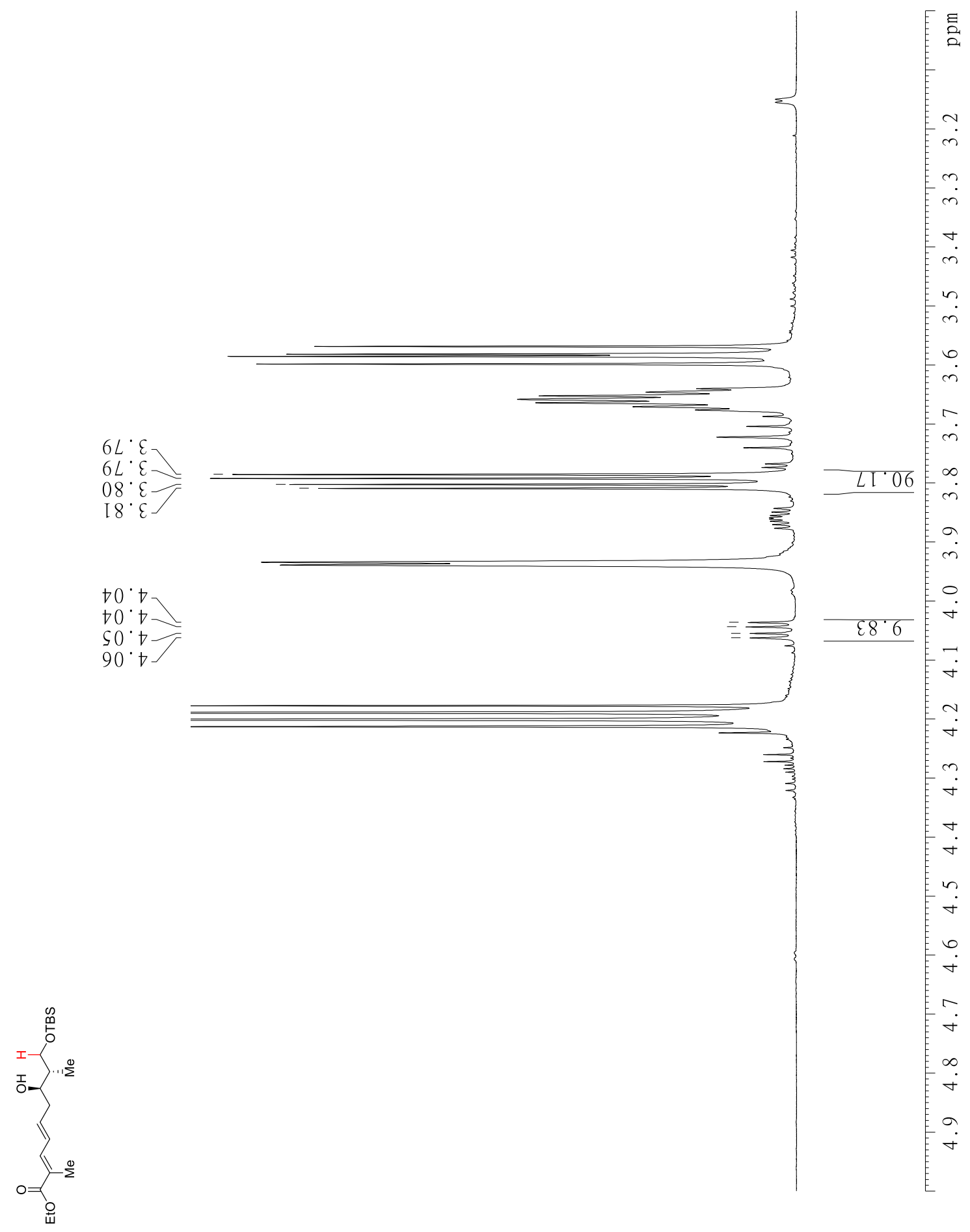

Spectrum 38. Enlarged view of the ${ }^{1} \mathrm{H}$ spectrum of $\mathbf{5 q}$ for the determination of the diastereomeric ratio; the spectrum was measured in $\mathrm{CDCl}_{3}$ at $600 \mathrm{MHz}$. 

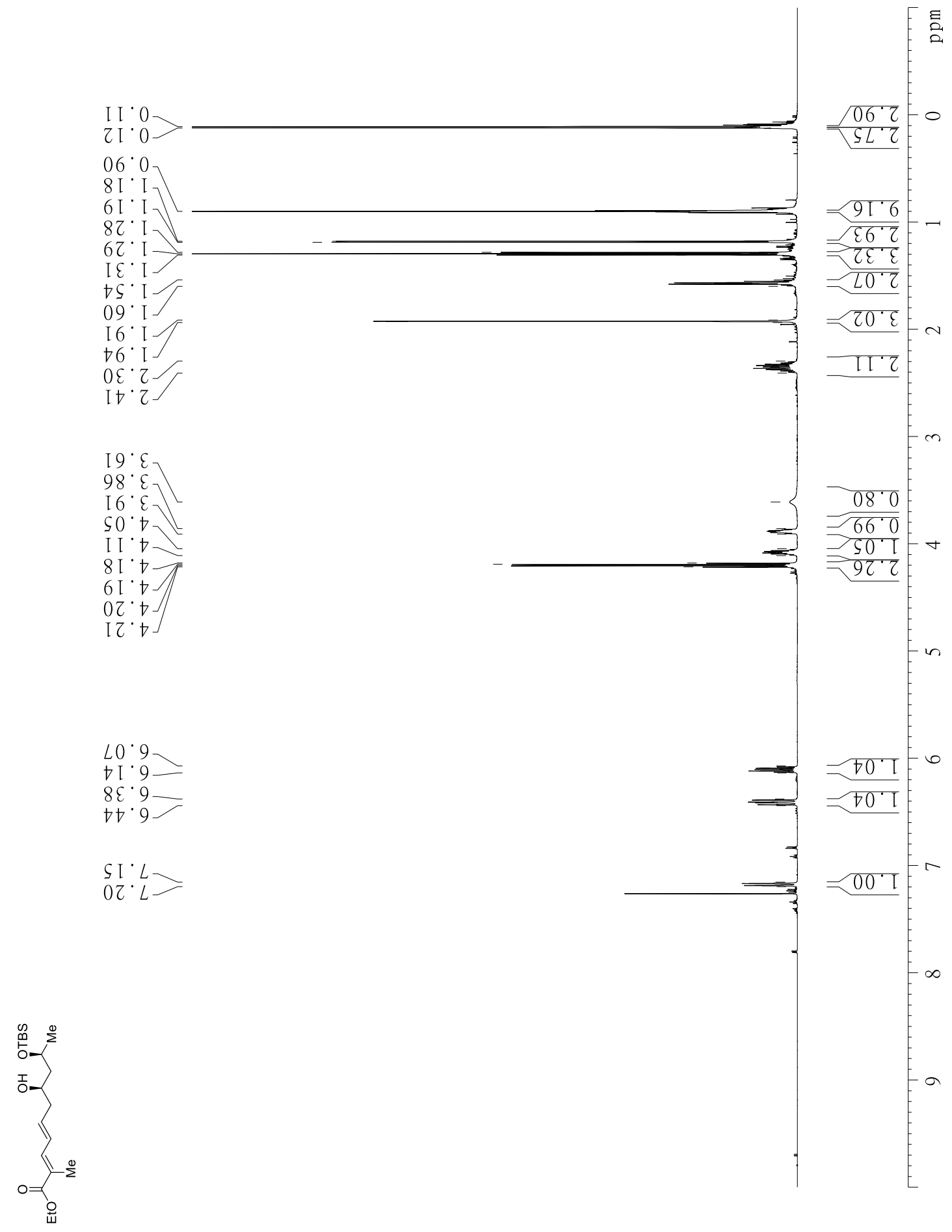

Spectrum 39. ${ }^{1} \mathrm{H}$ spectrum of $\mathbf{5 r}$ measured in $\mathrm{CDCl}_{3}$ at $400 \mathrm{MHz}$. 


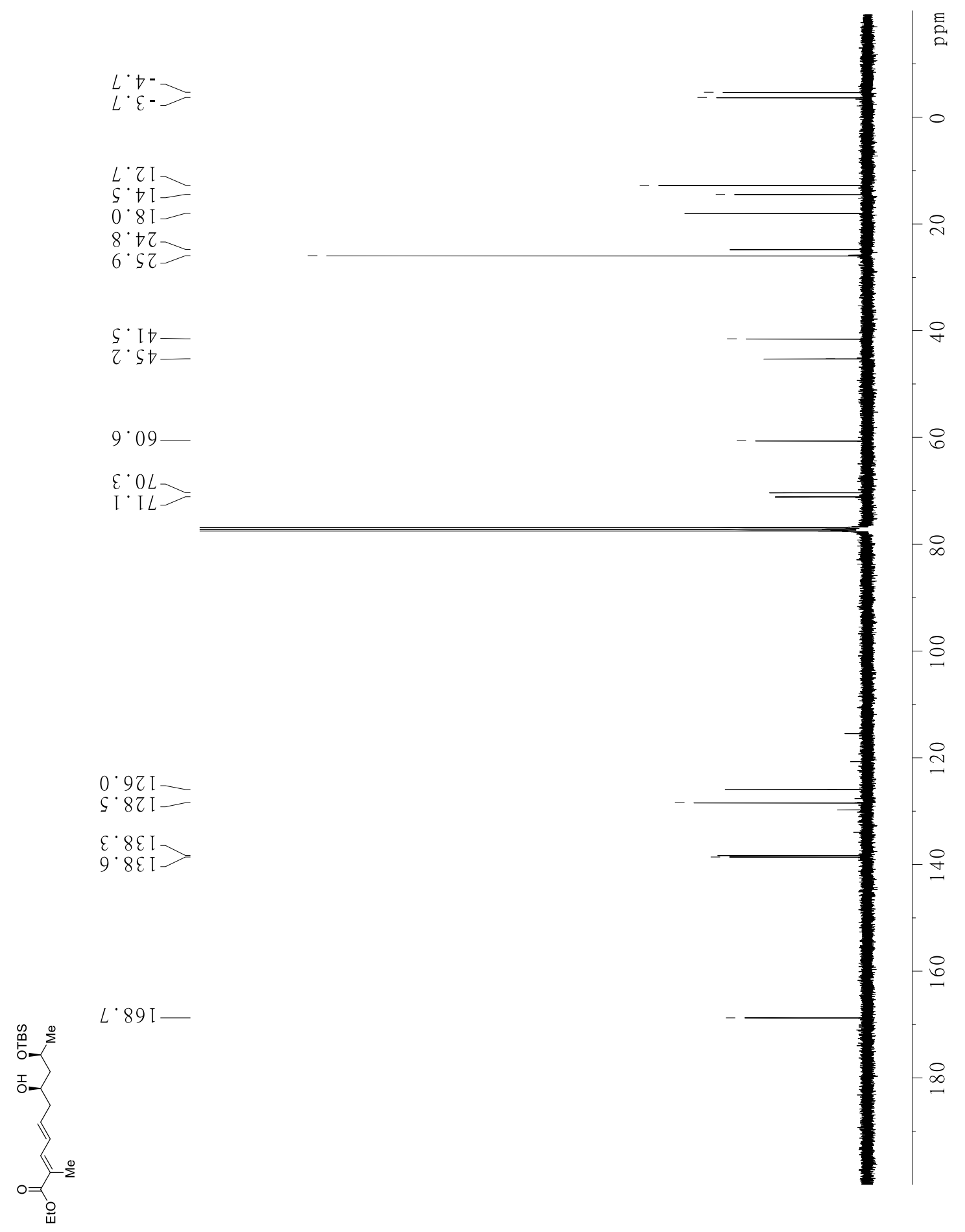

Spectrum 40. ${ }^{13} \mathrm{C}$ spectrum of $\mathbf{5 r}$ measured in $\mathrm{CDCl}_{3}$ at $101 \mathrm{MHz}$. 


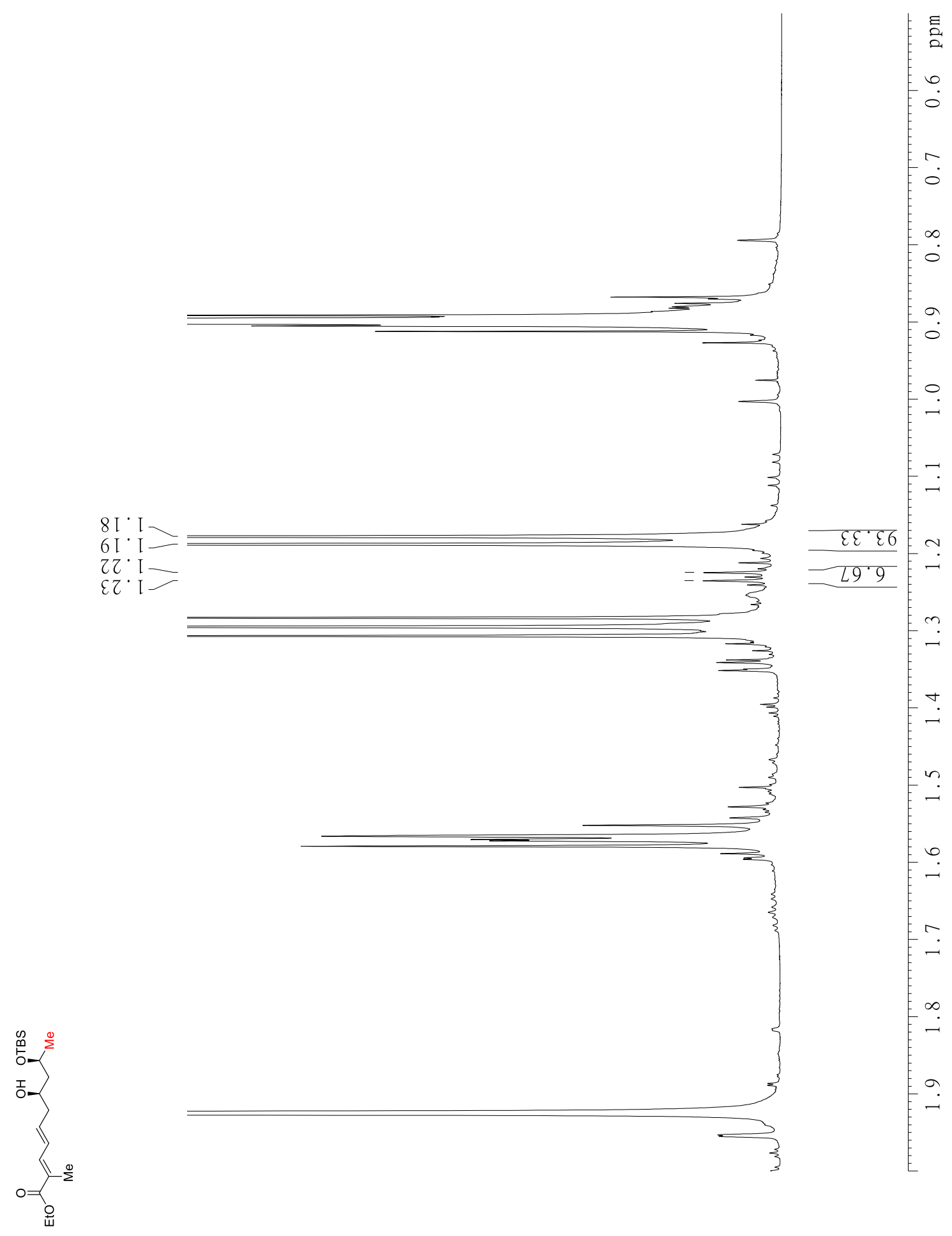

Spectrum 41. Enlarged view of the ${ }^{1} \mathrm{H}$ spectrum of $\mathbf{5 r}$ for the determination of the diastereomeric ratio; the spectrum was measured in $\mathrm{CDCl}_{3}$ at $600 \mathrm{MHz}$. 


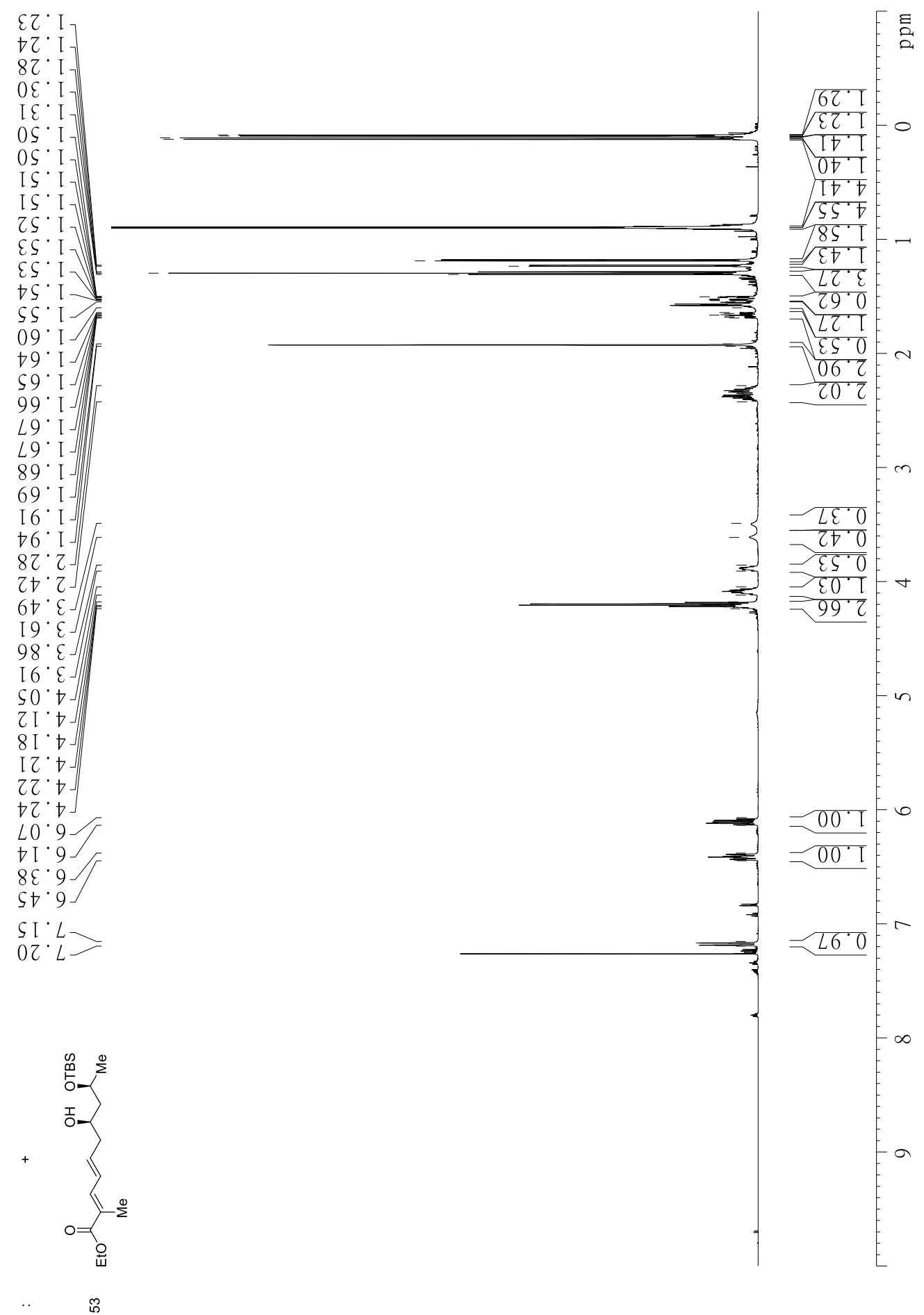

Spectrum 42. ${ }^{1} \mathrm{H}$ spectrum of $5 \mathrm{~s}$ measured in $\mathrm{CDCl}_{3}$ at $400 \mathrm{MHz}$. 5s was obtained as a mixture with its diastereomer $\mathbf{5 r}$. 
$\nabla^{\prime}$
$L$
$\varepsilon^{-}$

$8^{\circ} \mathrm{ZI}-$
$\mathrm{c}^{\circ} \cdot \mathrm{II}-$

$0.8 \mathrm{I}$

I. 8 I

$8 \cdot 27$

$8 \cdot \nabla z$

$6 \cdot 52$

$6^{\circ} \varsigma \mathrm{r}$

S. It

$8^{\circ}$ It

$8 \cdot \varepsilon t$

$\varepsilon \cdot \varsigma \downarrow$

$9^{\circ} 09$
$8^{\circ} \angle 9$

$8^{\circ} \angle 9$

$\varepsilon \cdot 0 L$

I. I $L$

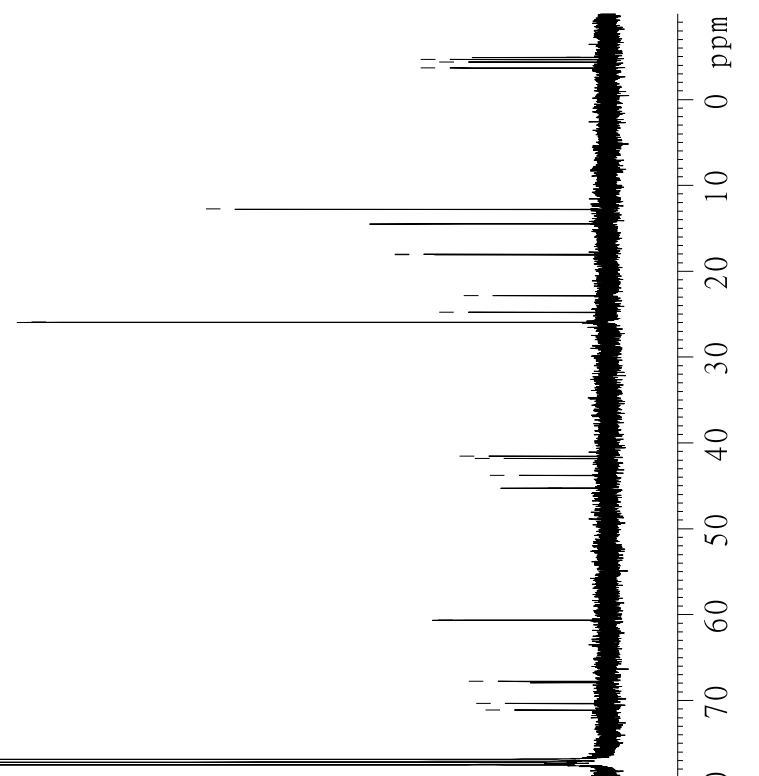

$\infty$

ㅇ

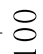

으

ำ

$0.97 \mathrm{I}$

0.921

t.8Z I

S.8Z I

$\varepsilon \cdot 8 \varepsilon \mathrm{I}$

$9 \cdot 8 \varepsilon \mathrm{I}$.

$L \cdot 8 \varepsilon I$

L.89I

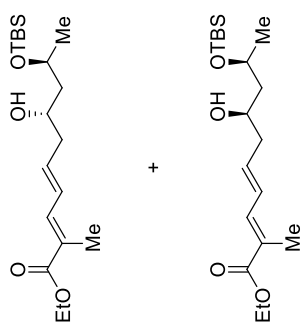

f $\quad . . \quad$ ถ

Spectrum 43. ${ }^{13} \mathrm{C}$ spectrum of $\mathbf{5 s}$ measured in $\mathrm{CDCl}_{3}$ at $101 \mathrm{MHz}$. $5 \mathbf{s}$ was obtained as a mixture with its diastereomer $\mathbf{5 r}$. 


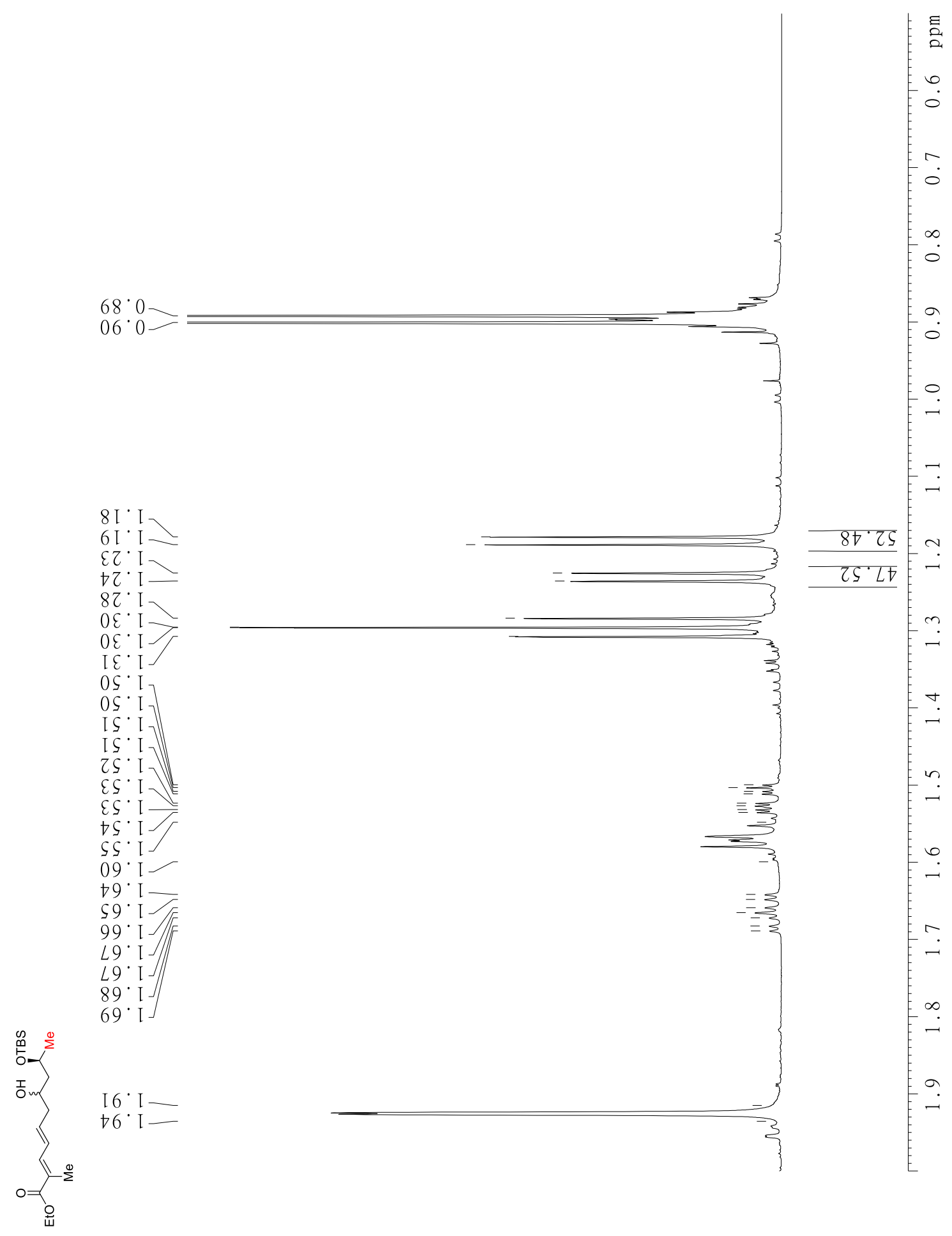

Spectrum 44. Enlarged view of the ${ }^{1} \mathrm{H}$ spectrum of $\mathbf{5 s}$ for the determination of the diastereomeric ratio; the spectrum was measured in $\mathrm{CDCl}_{3}$ at $600 \mathrm{MHz}$. 


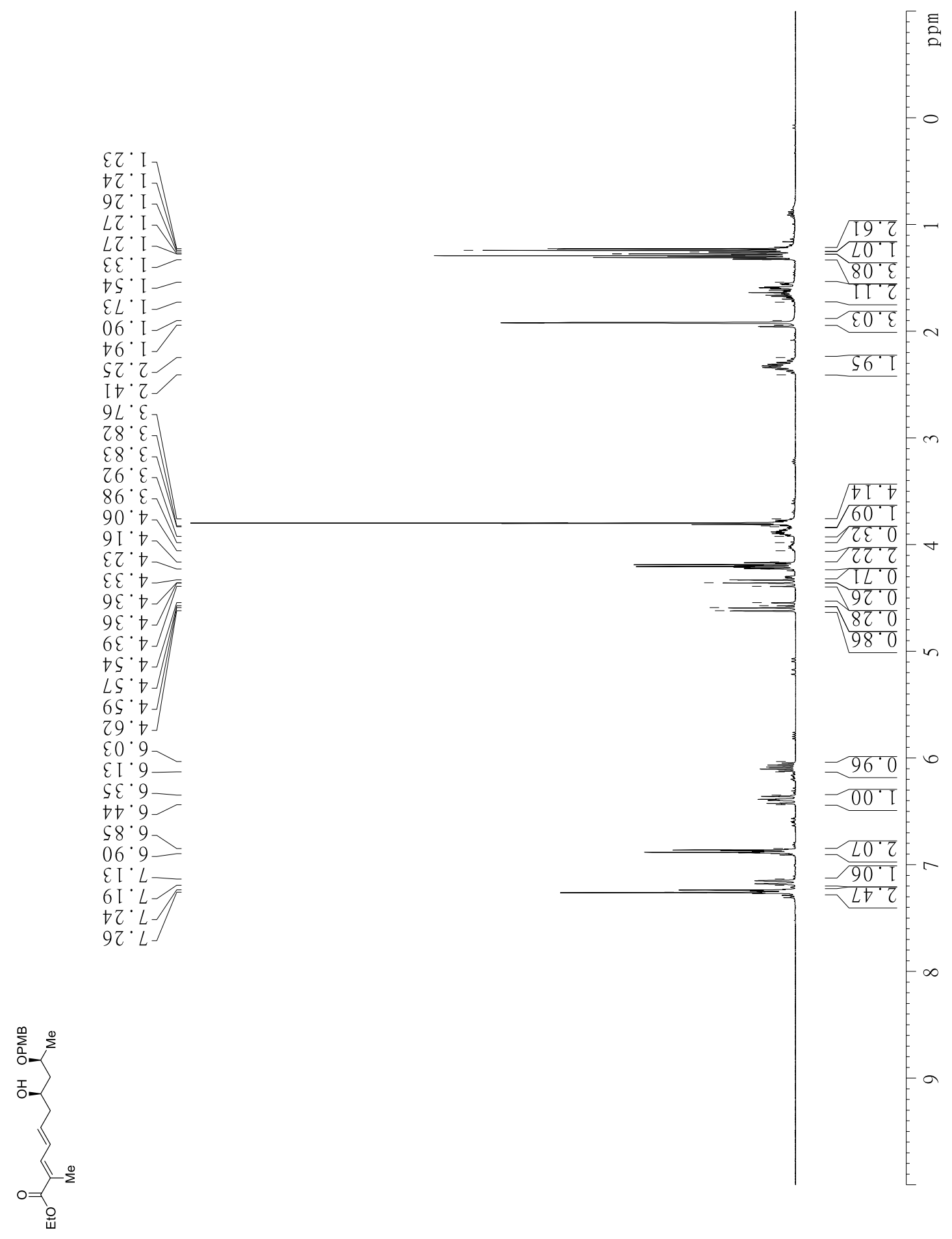

Spectrum 45. ${ }^{1} \mathrm{H}$ spectrum of $\mathbf{5 t}$ measured in $\mathrm{CDCl}_{3}$ at $400 \mathrm{MHz}$. 


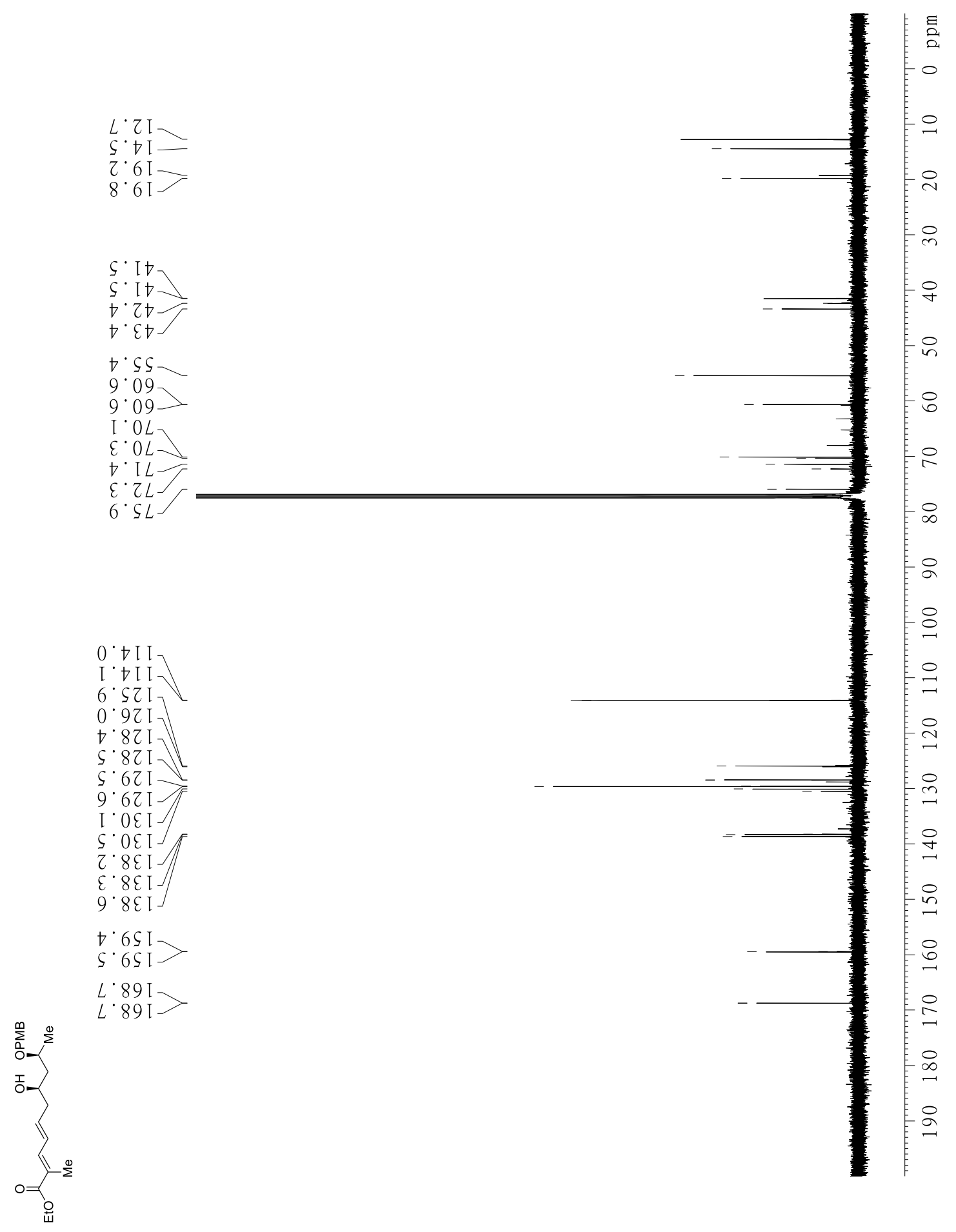

Spectrum 46. ${ }^{13} \mathrm{C}$ spectrum of $\mathbf{5 t}$ measured in $\mathrm{CDCl}_{3}$ at $101 \mathrm{MHz}$. 


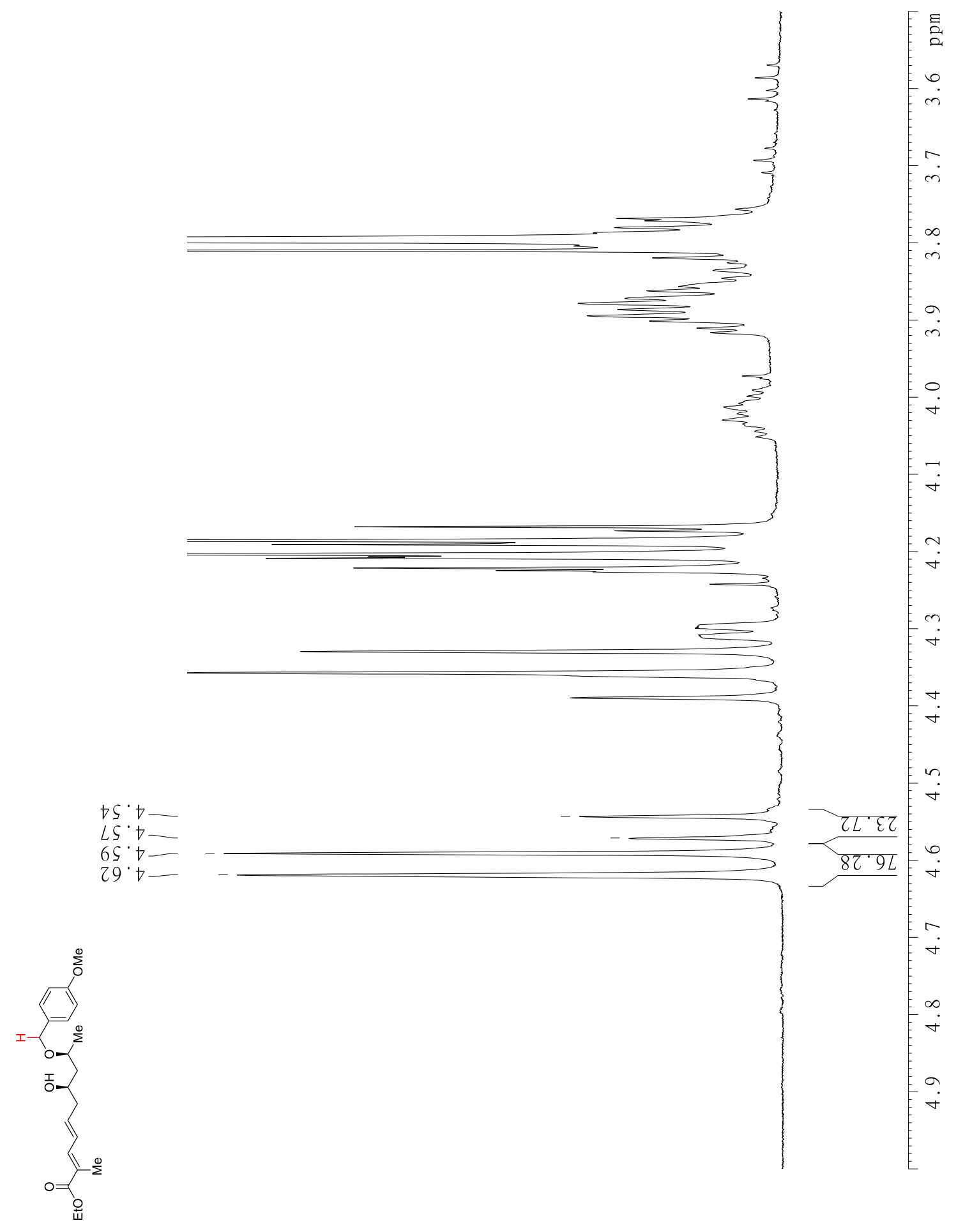

Spectrum 47. Enlarged view of the ${ }^{1} \mathrm{H}$ spectrum of $\mathbf{5 t}$ for the determination of the diastereomeric ratio; the spectrum was measured in $\mathrm{CDCl}_{3}$ at $400 \mathrm{MHz}$. 


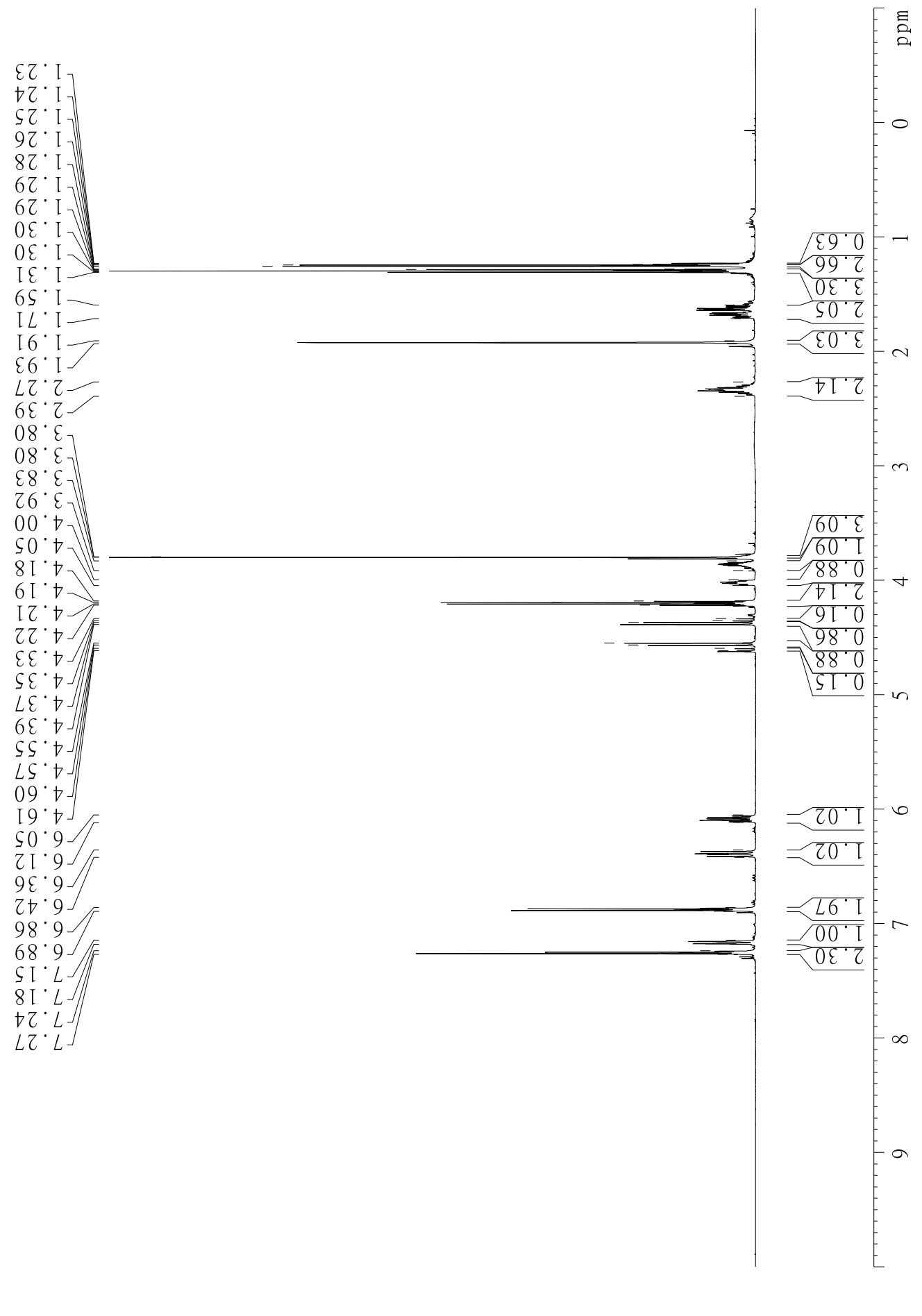

Spectrum 48. ${ }^{1} \mathrm{H}$ spectrum of $5 \mathbf{u}$ measured in $\mathrm{CDCl}_{3}$ at $600 \mathrm{MHz}$. 


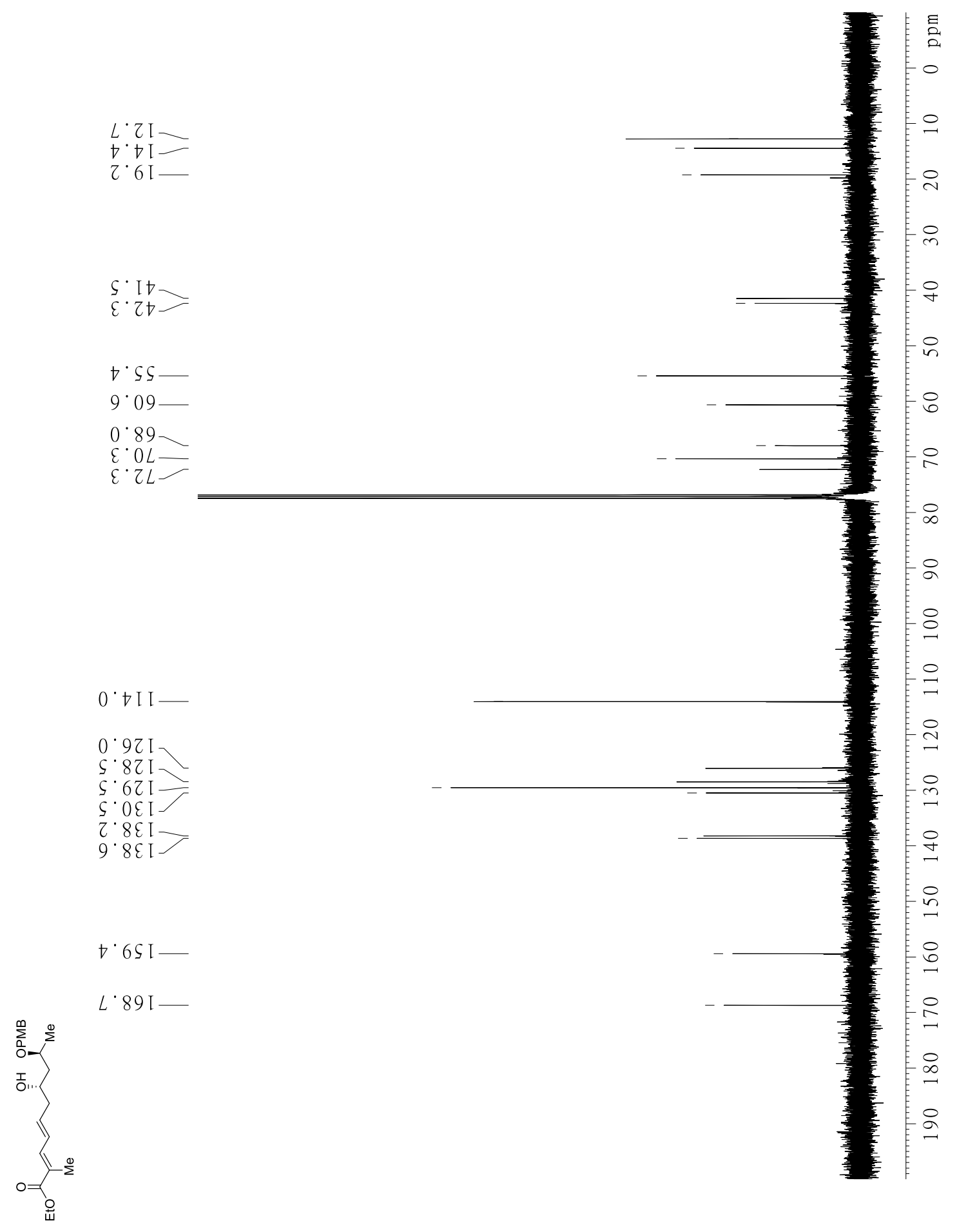

Spectrum 49. ${ }^{13} \mathrm{C}$ spectrum of $\mathbf{5 u}$ measured in $\mathrm{CDCl}_{3}$ at $101 \mathrm{MHz}$. 


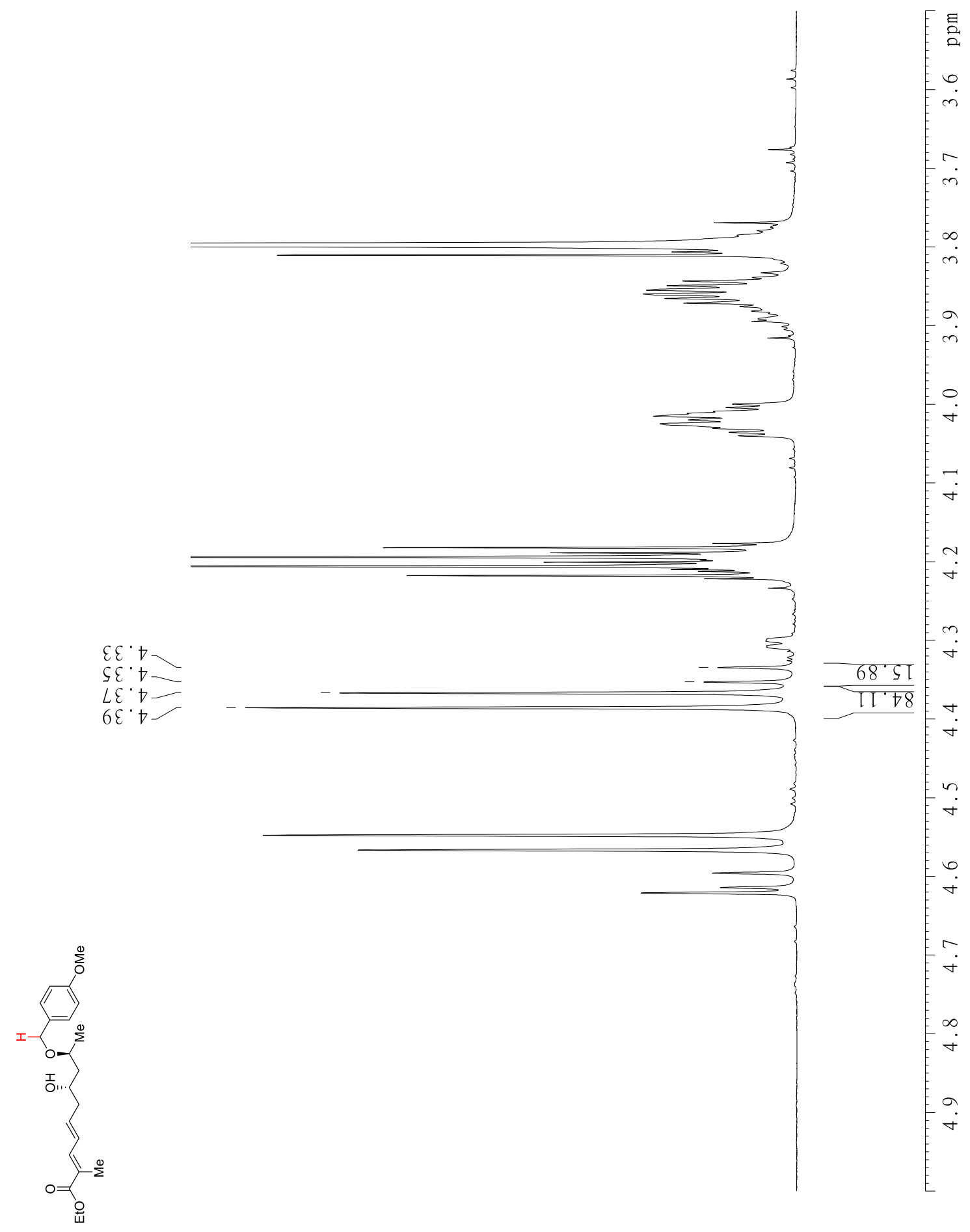

Spectrum 50. Enlarged view of the ${ }^{1} \mathrm{H}$ spectrum of $\mathbf{5 u}$ for the determination of the diastereomeric ratio; the spectrum was measured in $\mathrm{CDCl}_{3}$ at $600 \mathrm{MHz}$. 


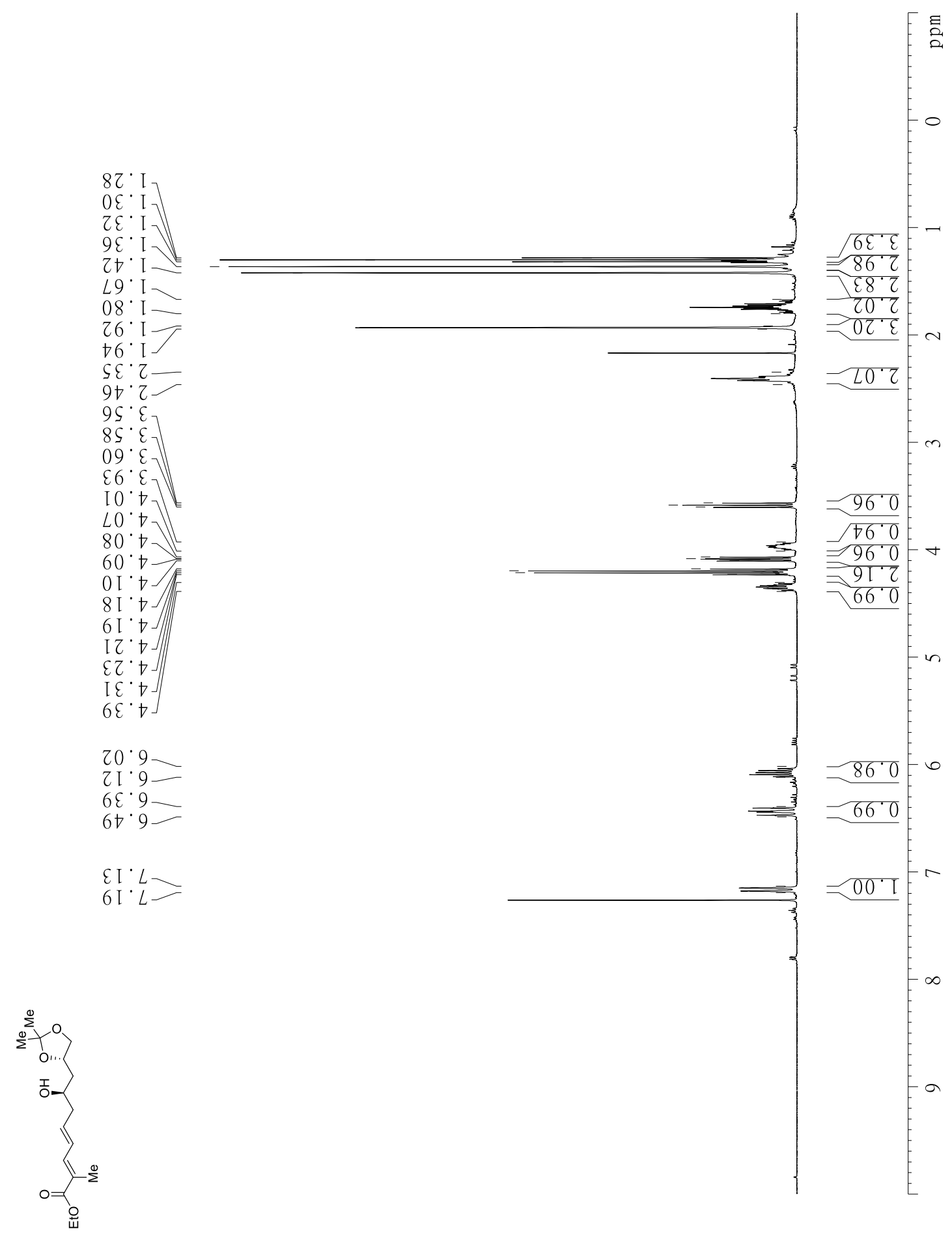

Spectrum 51. ${ }^{1} \mathrm{H}$ spectrum of $\mathbf{5 v}$ measured in $\mathrm{CDCl}_{3}$ at $400 \mathrm{MHz}$. 


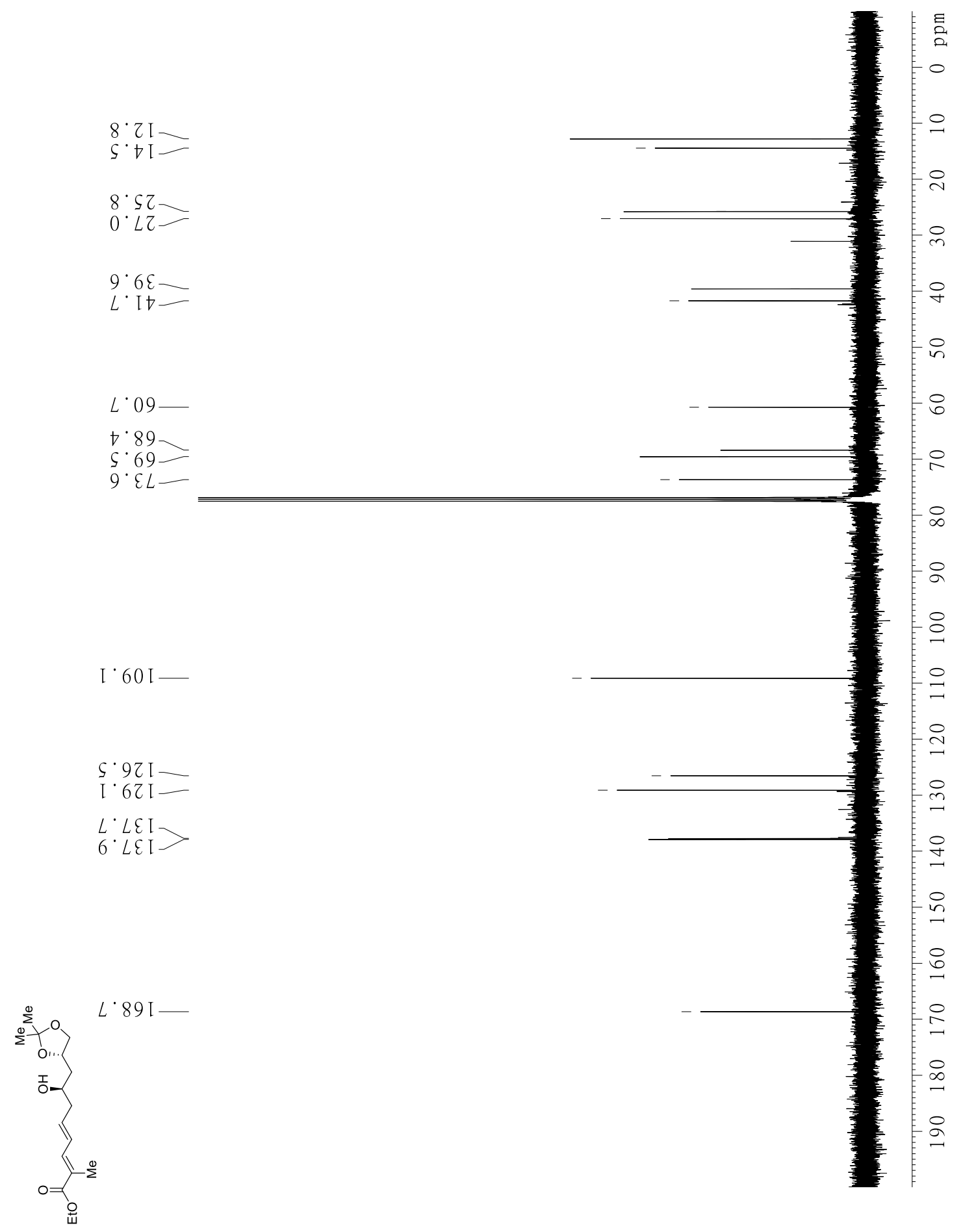

Spectrum 52. ${ }^{13} \mathrm{C}$ spectrum of $\mathbf{5 v}$ measured in $\mathrm{CDCl}_{3}$ at $101 \mathrm{MHz}$. 


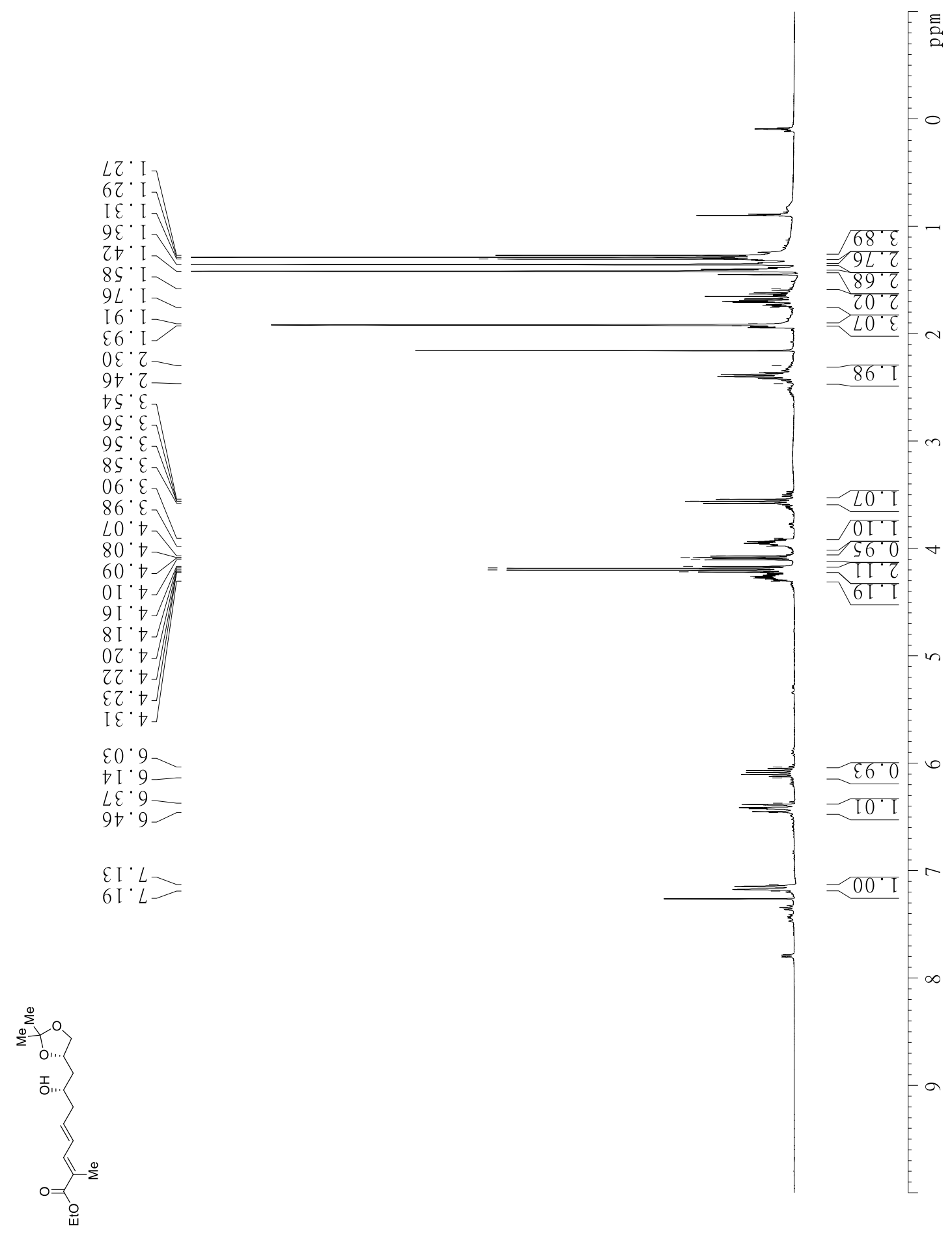

Spectrum 53. ${ }^{1} \mathrm{H}$ spectrum of $\mathbf{5 w}$ measured in $\mathrm{CDCl}_{3}$ at $400 \mathrm{MHz}$. 


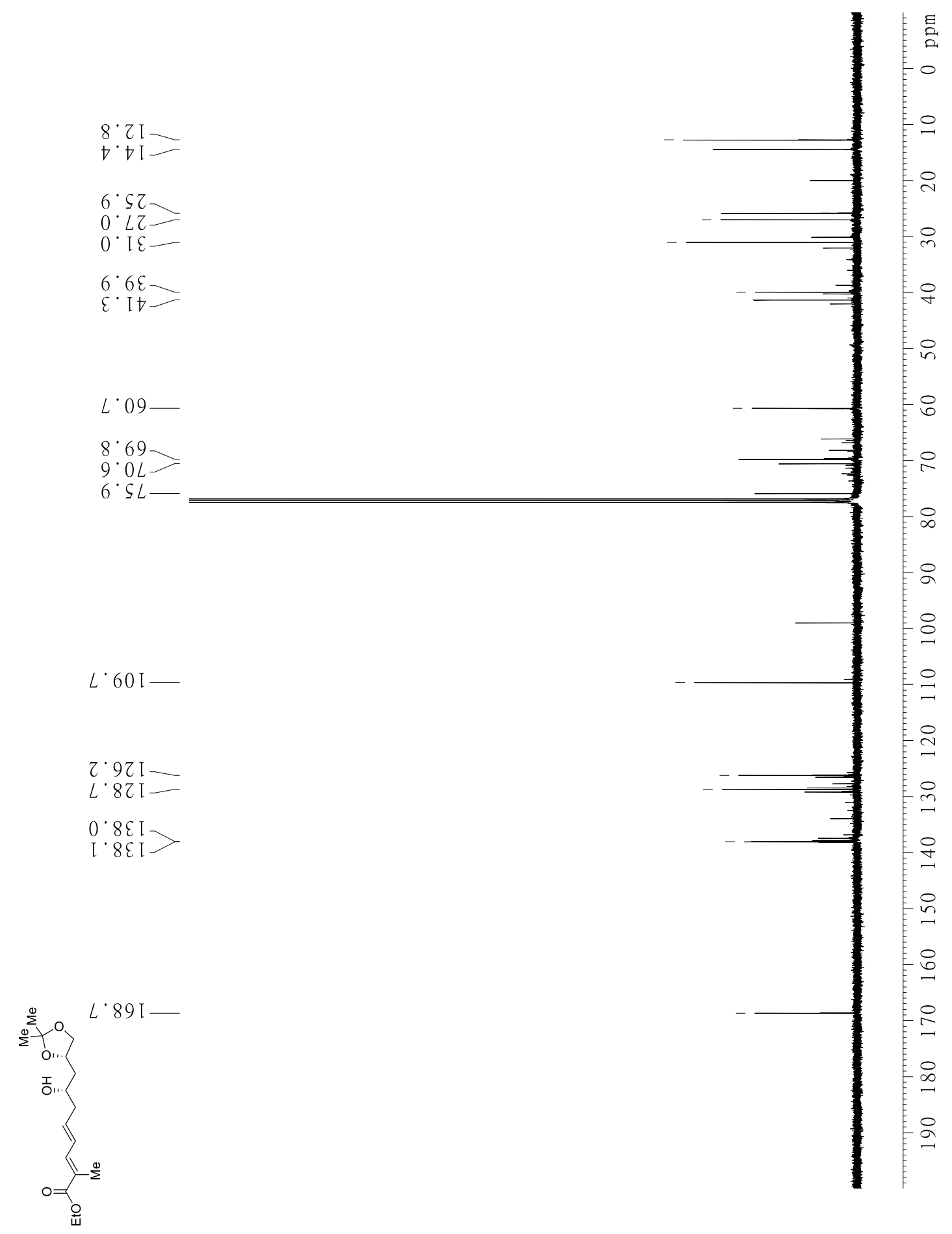

Spectrum 54. ${ }^{13} \mathrm{C}$ spectrum of $\mathbf{5 w}$ measured in $\mathrm{CDCl}_{3}$ at $101 \mathrm{MHz}$. 


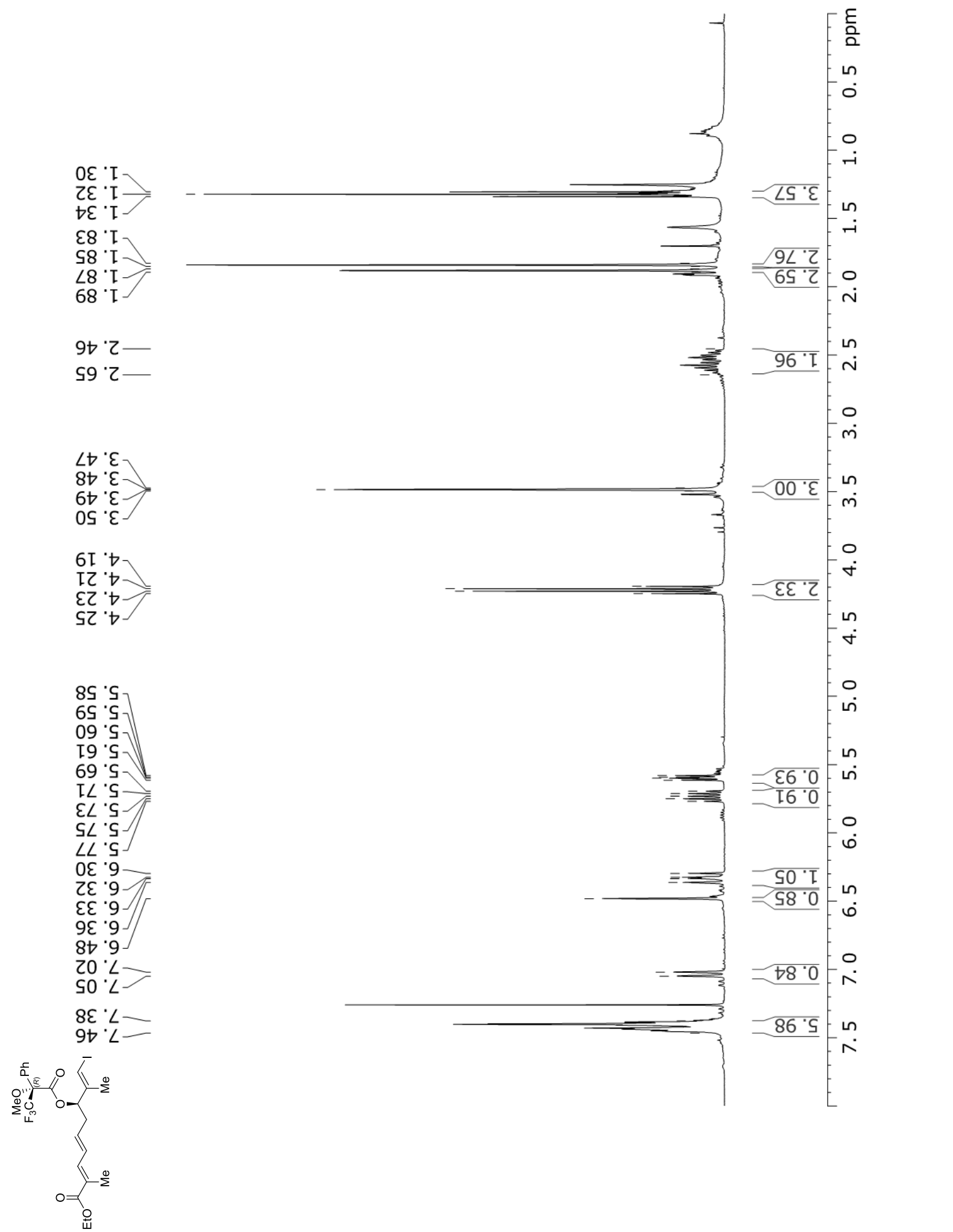

Spectrum 55. ${ }^{1} \mathrm{H}$ spectrum of the $(R)$-Mosher's ester of bVMAR product $\mathbf{5 g}$ measured in $\mathrm{CDCl}_{3}$ at $400 \mathrm{MHz}$. 


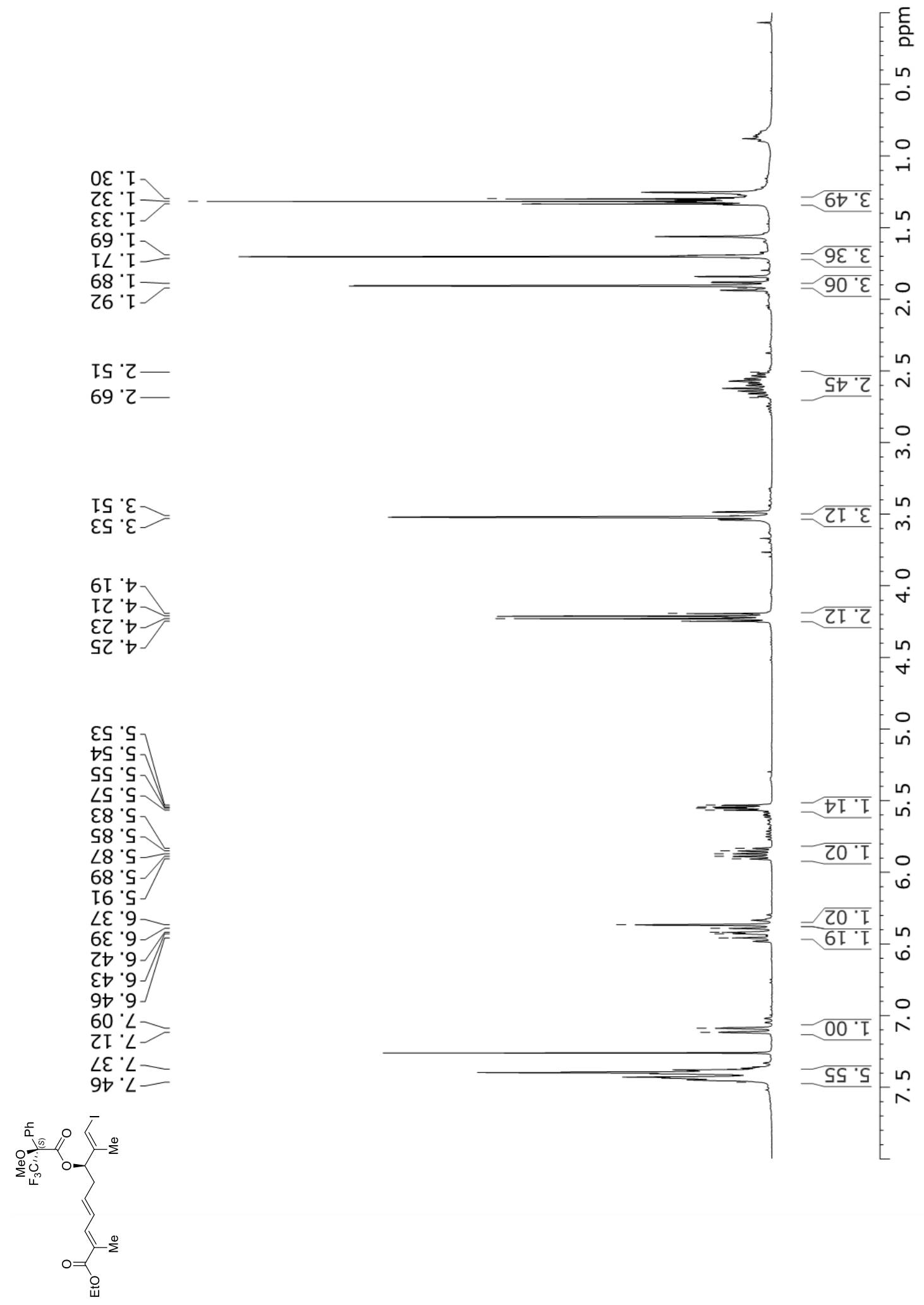

Spectrum 56. ${ }^{1} \mathrm{H}$ spectrum of the $(S)$-Mosher's ester of bVMAR product $5 \mathbf{g}$ measured in $\mathrm{CDCl}_{3}$ at $400 \mathrm{MHz}$. 


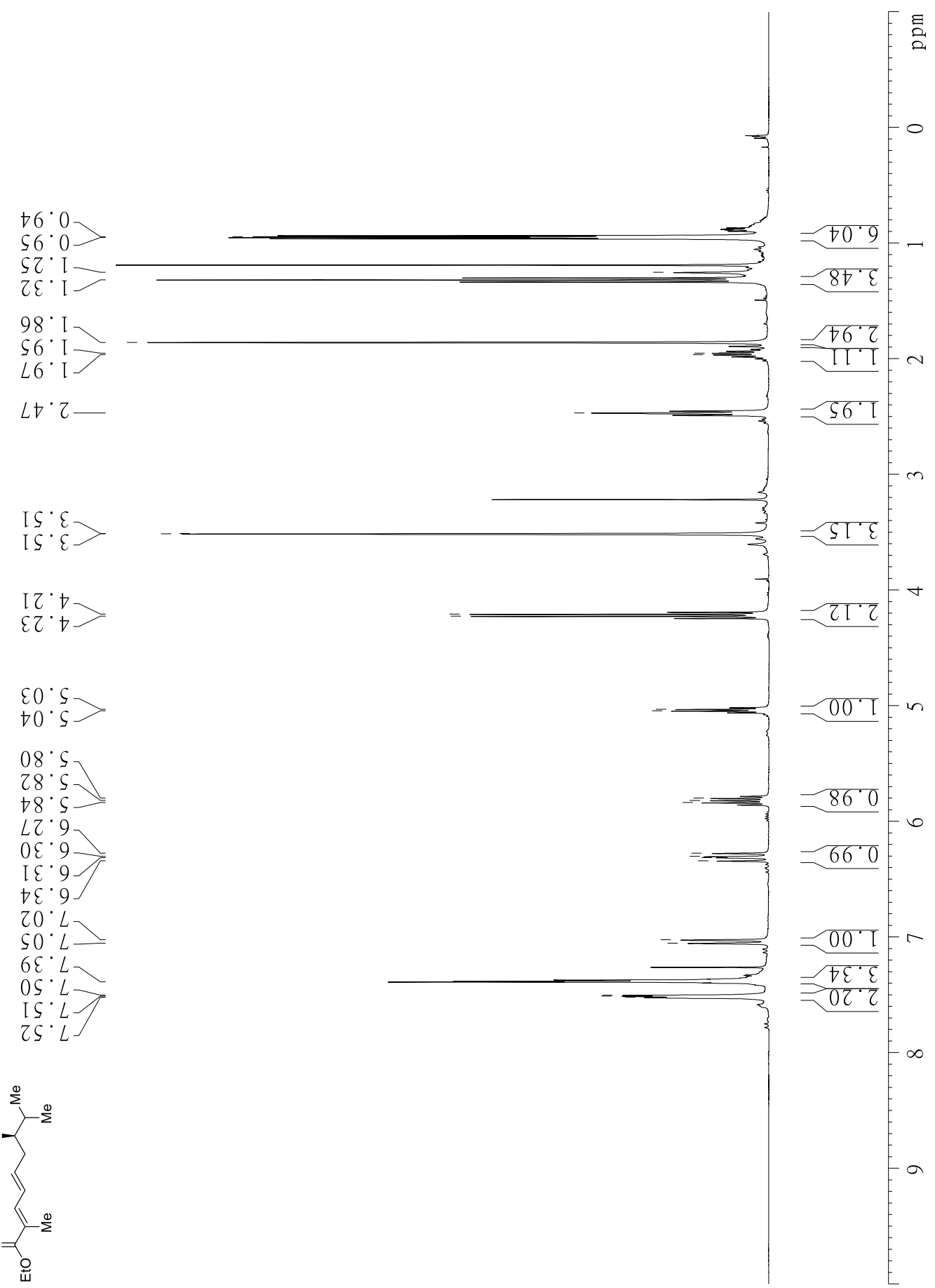

Spectrum 57. ${ }^{1} \mathrm{H}$ spectrum of the $(R)$-Mosher's ester of bVMAR product $5 \mathbf{k}$ measured in $\mathrm{CDCl}_{3}$ at $400 \mathrm{MHz}$. 


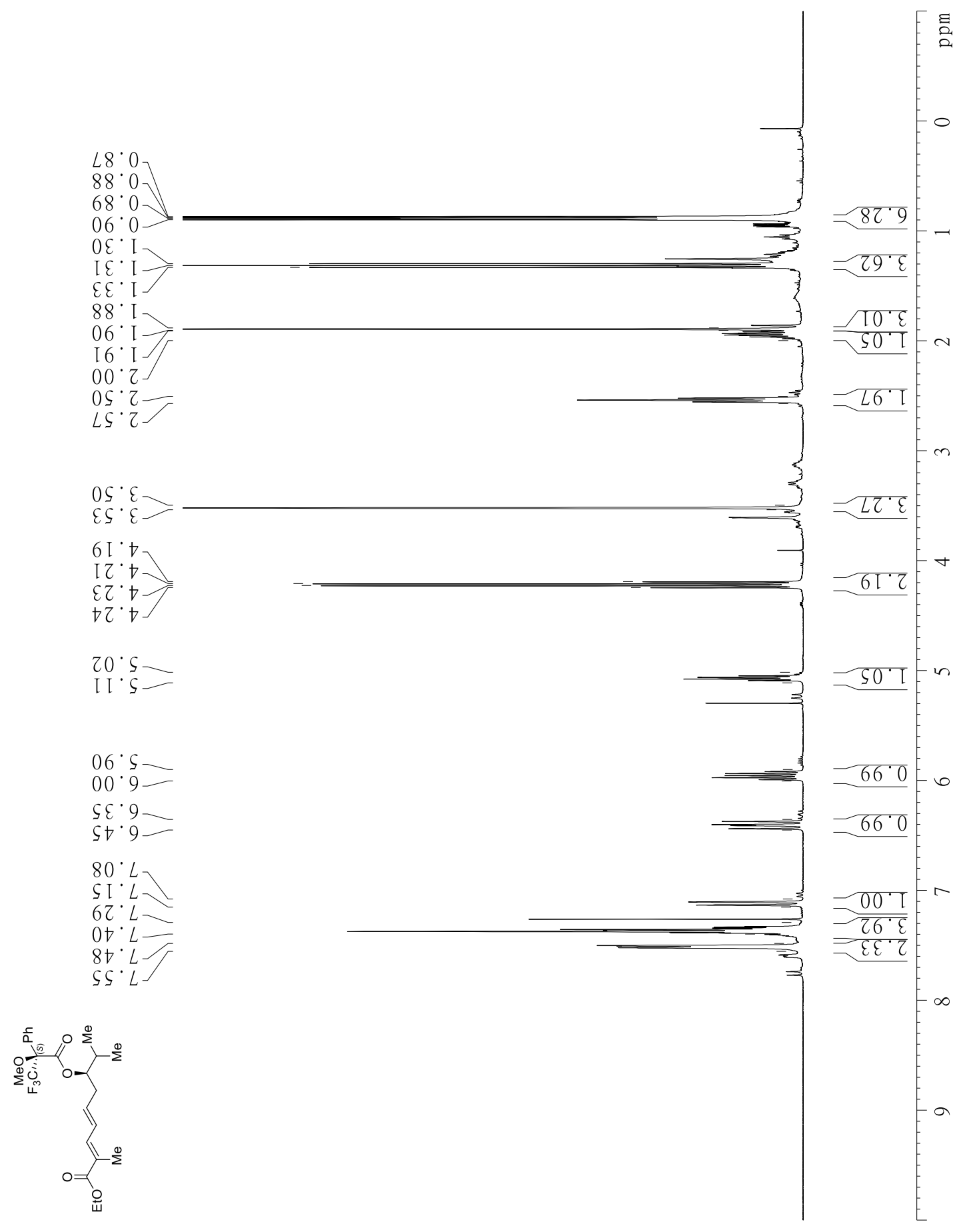

Spectrum 58. ${ }^{1} \mathrm{H}$ spectrum of the $(S)$-Mosher's ester of bVMAR product $\mathbf{5 k}$ measured in $\mathrm{CDCl}_{3}$ at $400 \mathrm{MHz}$. 


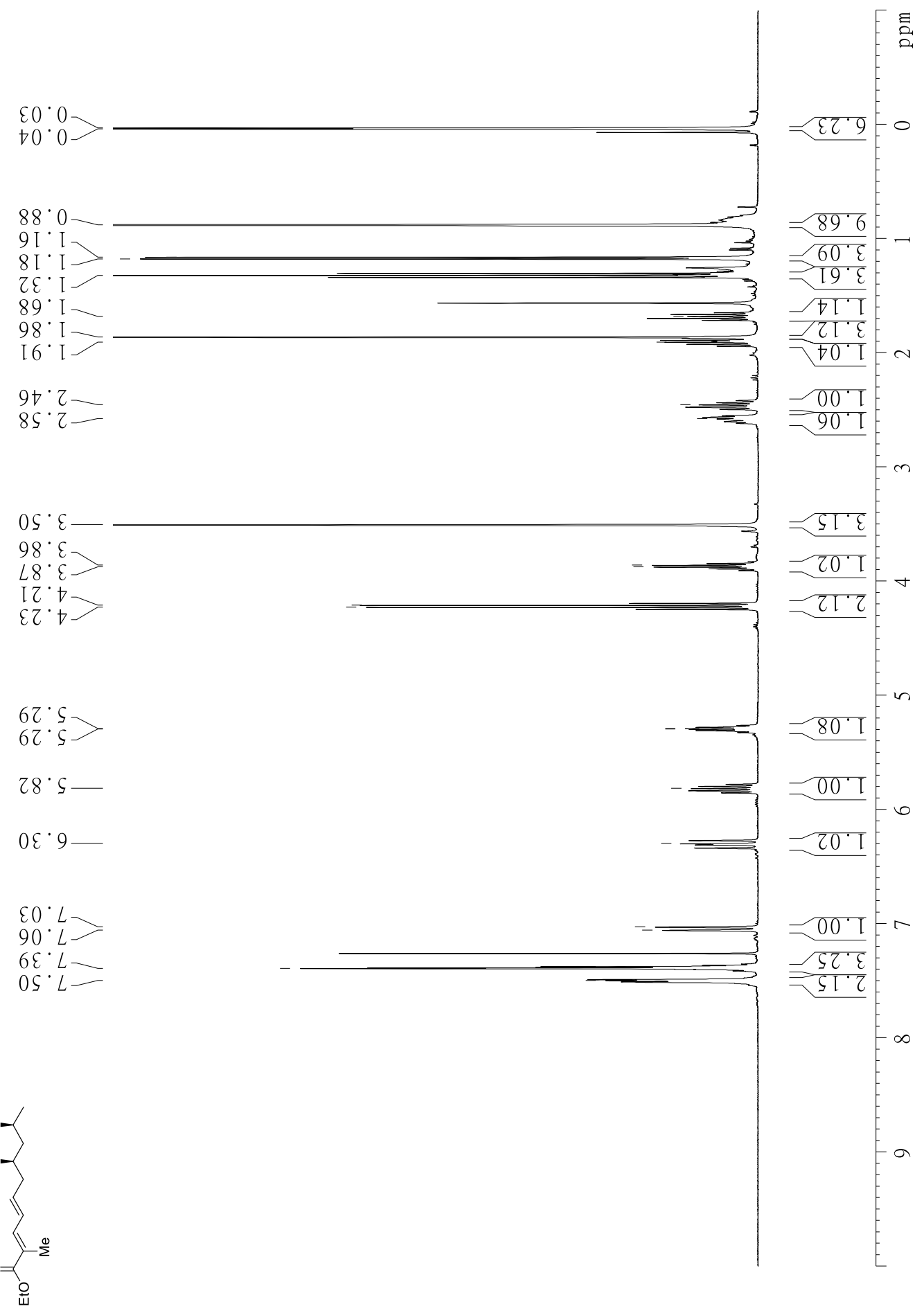

Spectrum 59. ${ }^{1} \mathrm{H}$ spectrum of the $(R)$-Mosher's ester of bVMAR product $5 \mathbf{r}$ measured in $\mathrm{CDCl}_{3}$ at $400 \mathrm{MHz}$. 


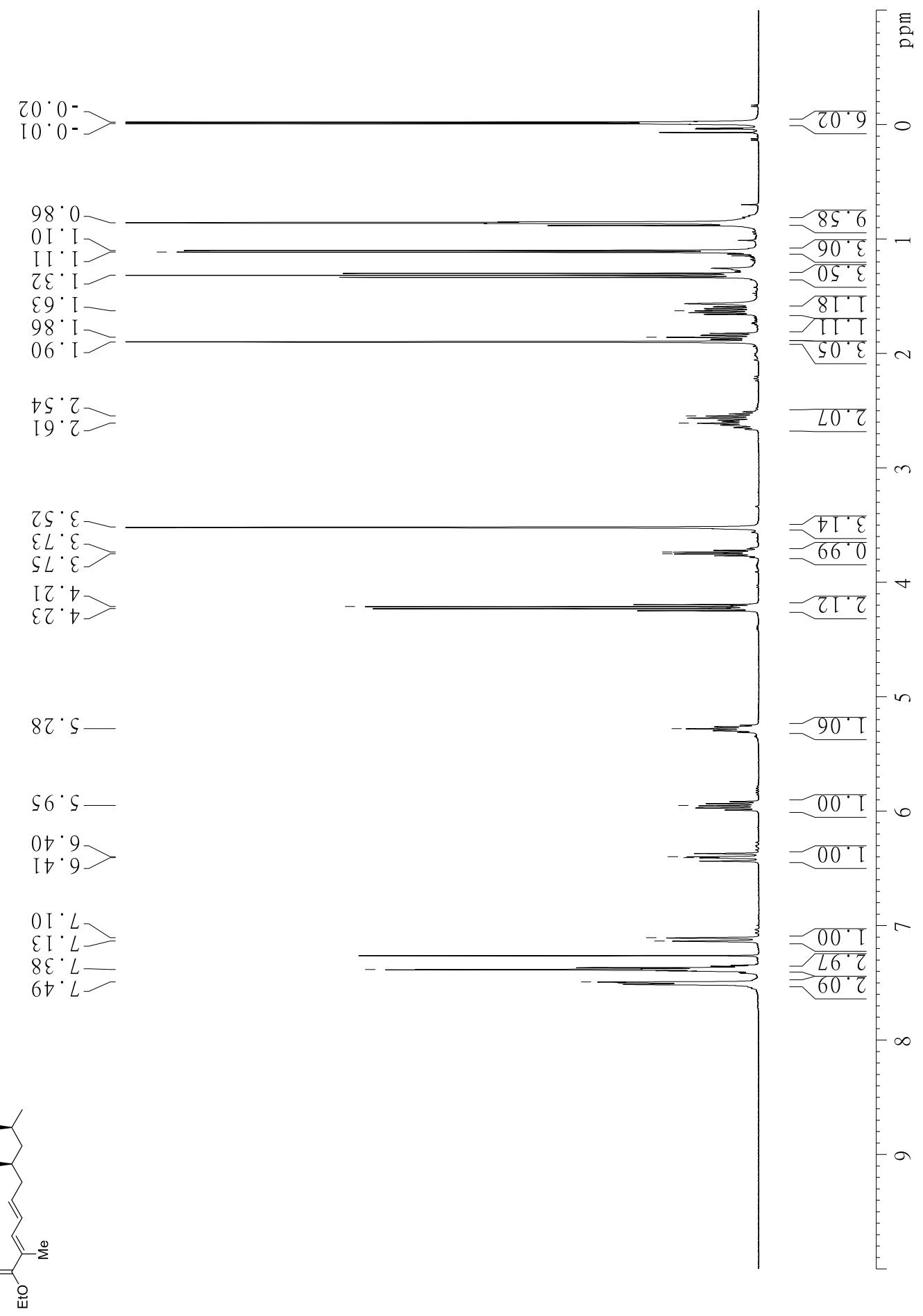

Spectrum 60. ${ }^{1} \mathrm{H}$ spectrum of the $(S)$-Mosher's ester of bVMAR product $5 \mathbf{r}$ measured in $\mathrm{CDCl}_{3}$ at $400 \mathrm{MHz}$. 


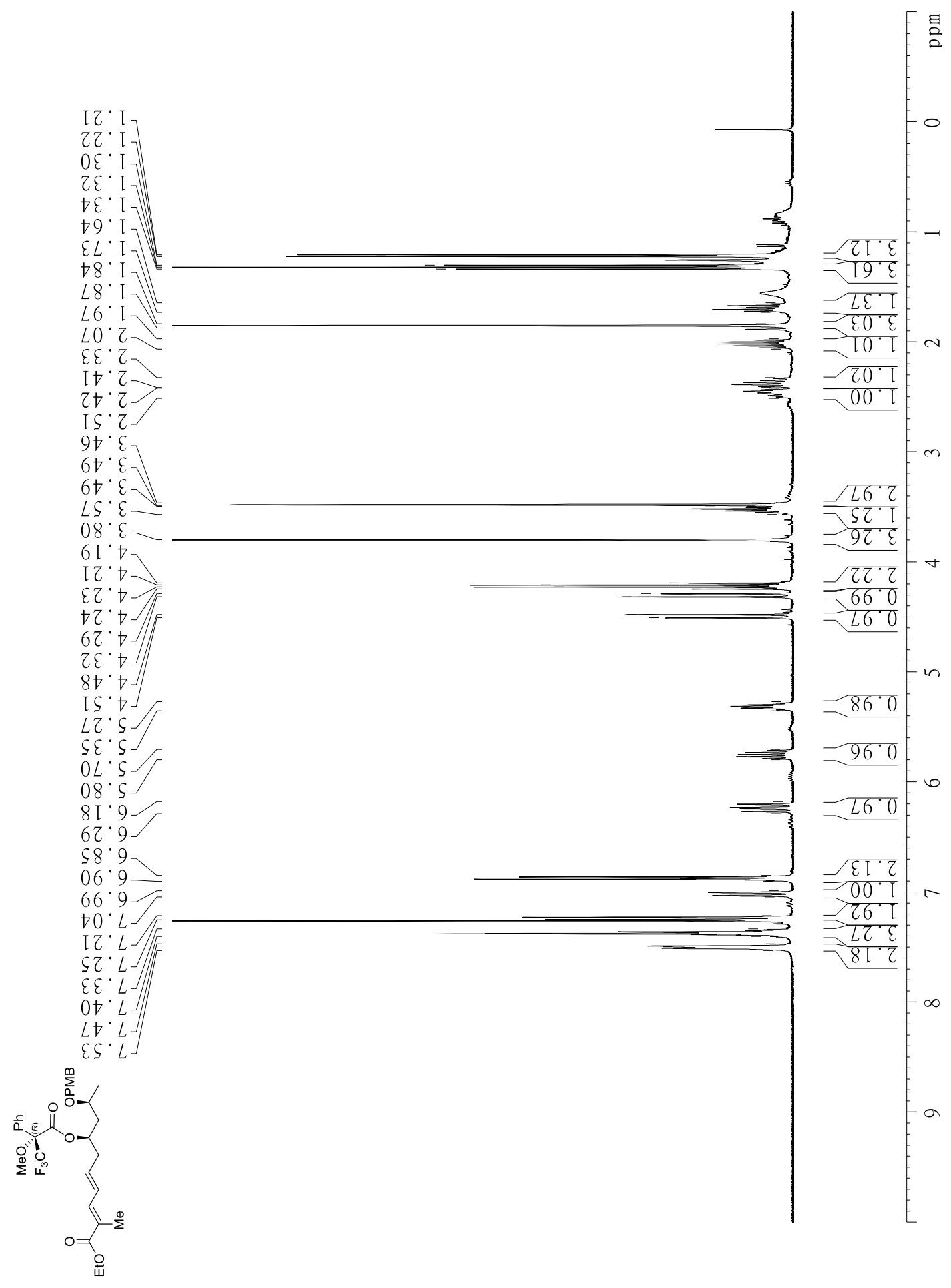

Spectrum 61. ${ }^{1} \mathrm{H}$ spectrum of the $(R)$-Mosher's ester of bVMAR product $5 \mathrm{t}$ measured in $\mathrm{CDCl}_{3}$ at $400 \mathrm{MHz}$. 


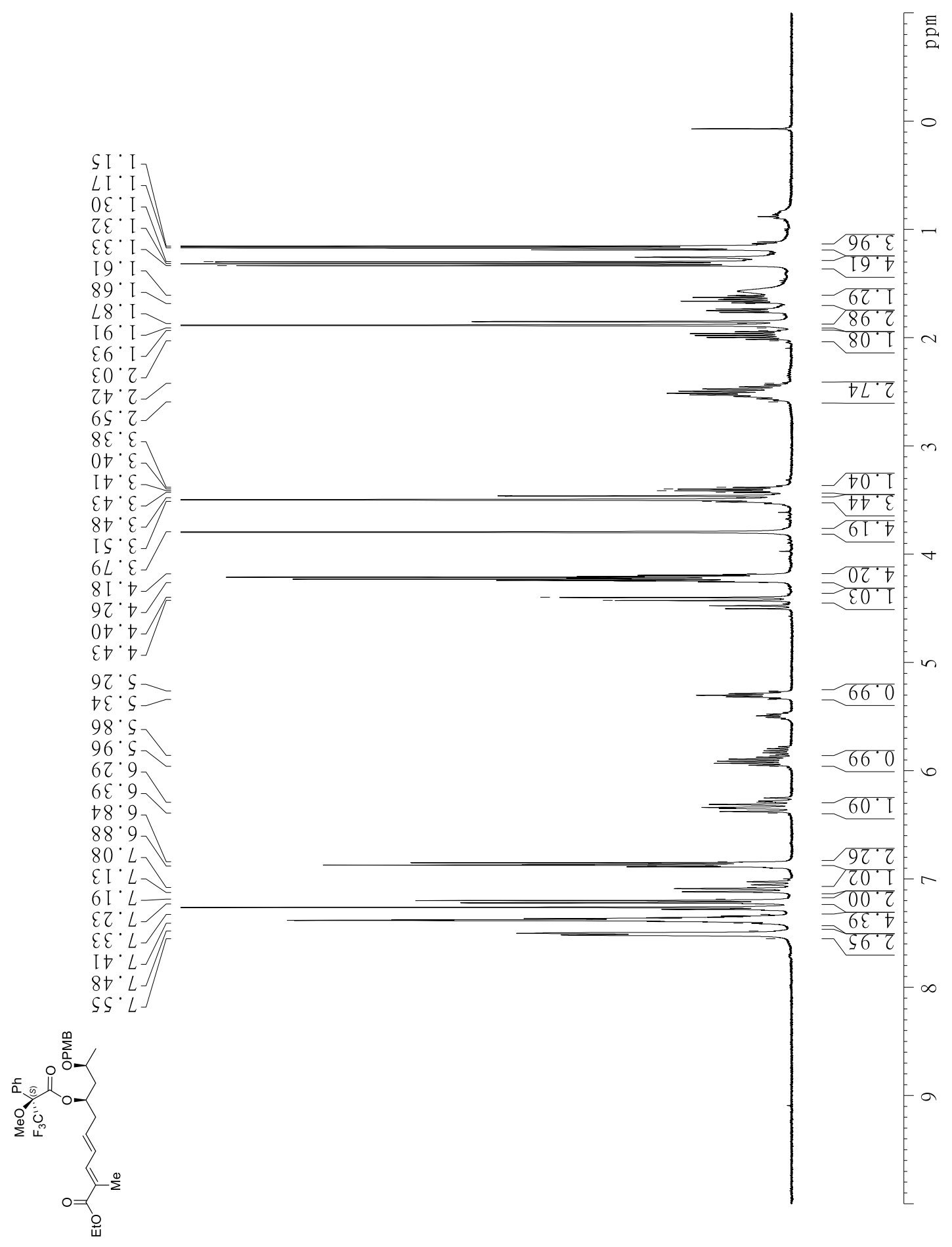

Spectrum 62. ${ }^{1} \mathrm{H}$ spectrum of the $(S)$-Mosher's ester of bVMAR product $\mathbf{5 t}$ measured in $\mathrm{CDCl}_{3}$ at $400 \mathrm{MHz}$. 


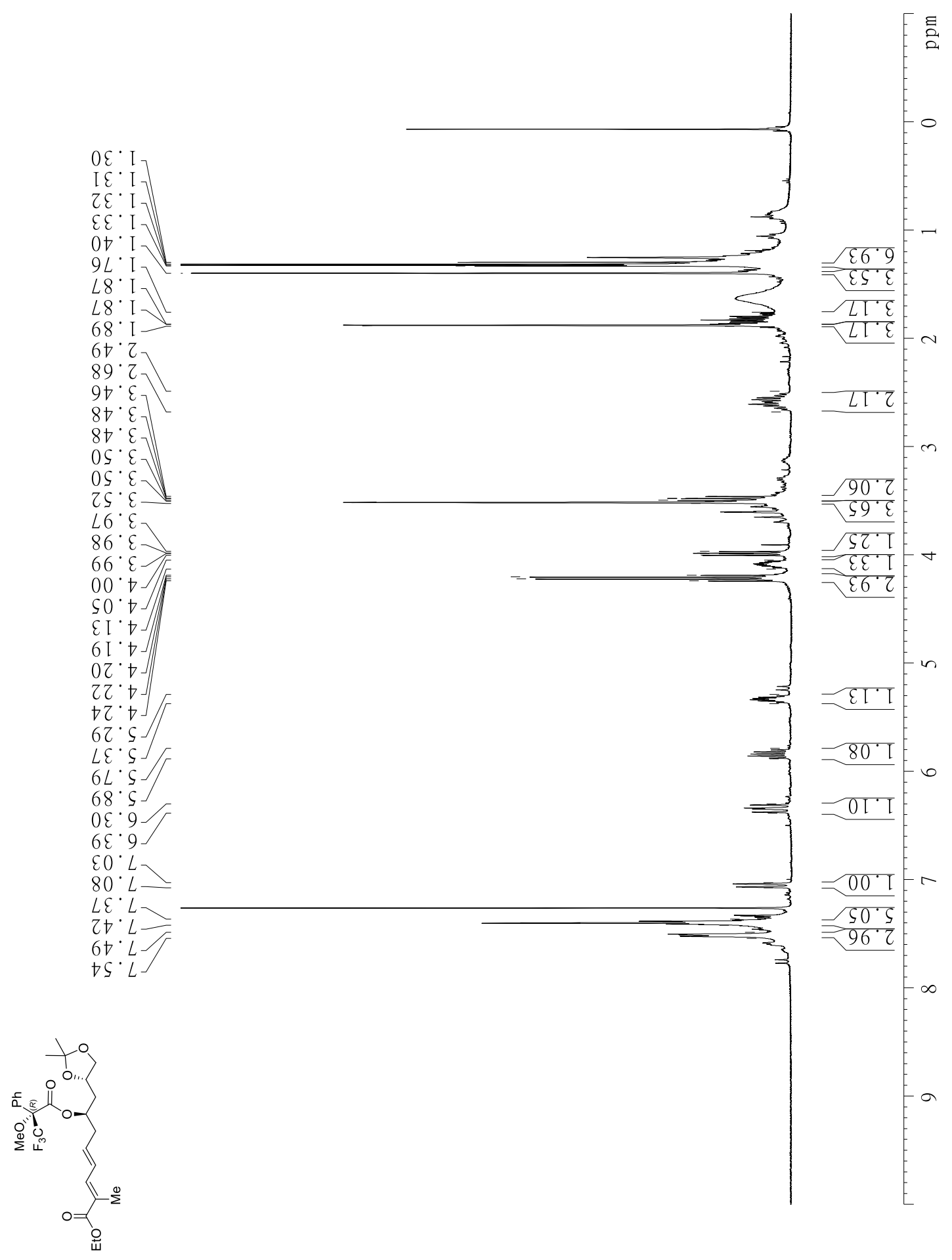

Spectrum 63. ${ }^{1} \mathrm{H}$ spectrum of the $(R)$-Mosher's ester of bVMAR product $\mathbf{5} \mathbf{v}$ measured in $\mathrm{CDCl}_{3}$ at $400 \mathrm{MHz}$. 


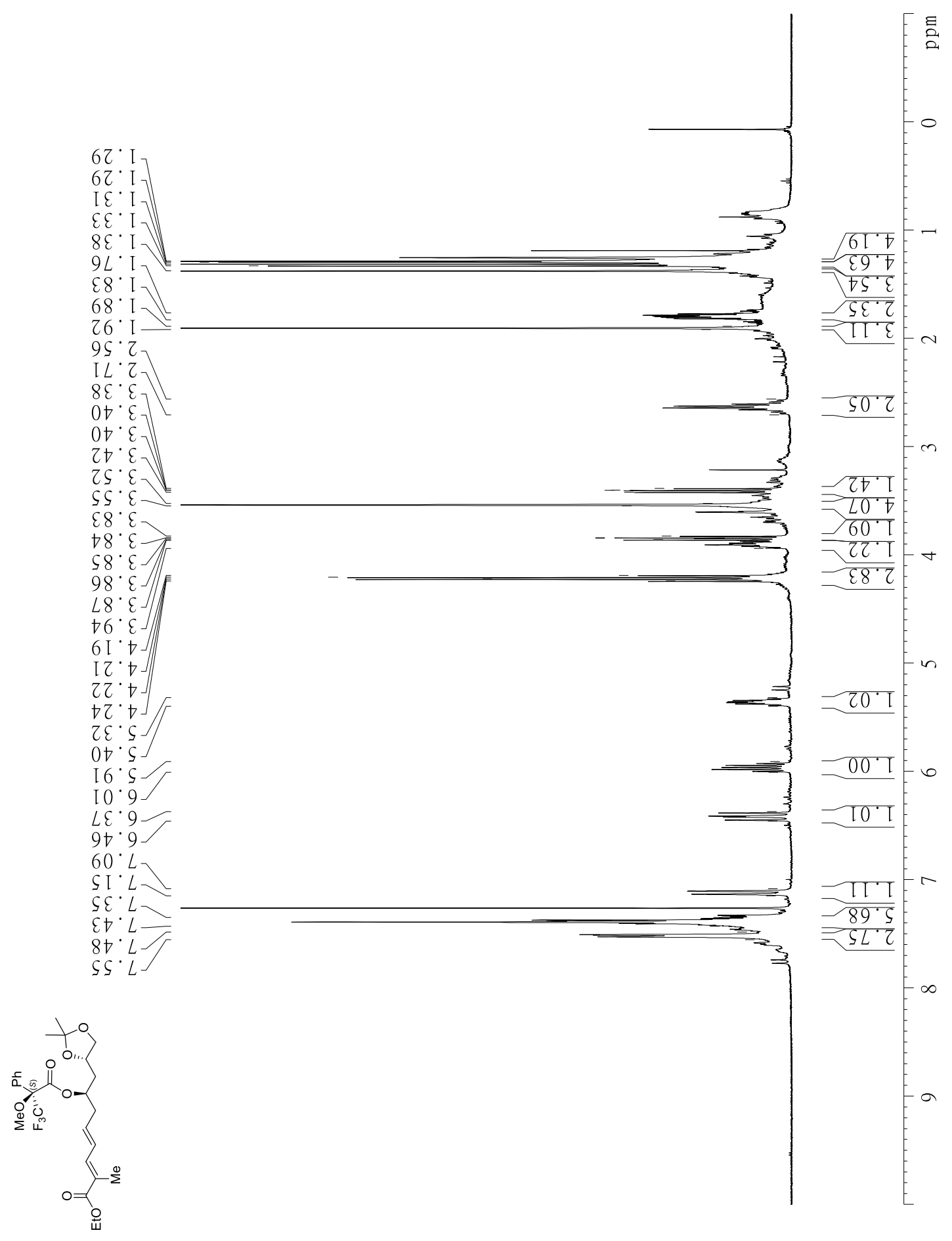

Spectrum 64. ${ }^{1} \mathrm{H}$ spectrum of the $(S)$-Mosher's ester of bVMAR product $\mathbf{5} \mathbf{v}$ measured in $\mathrm{CDCl}_{3}$ at $400 \mathrm{MHz}$. 


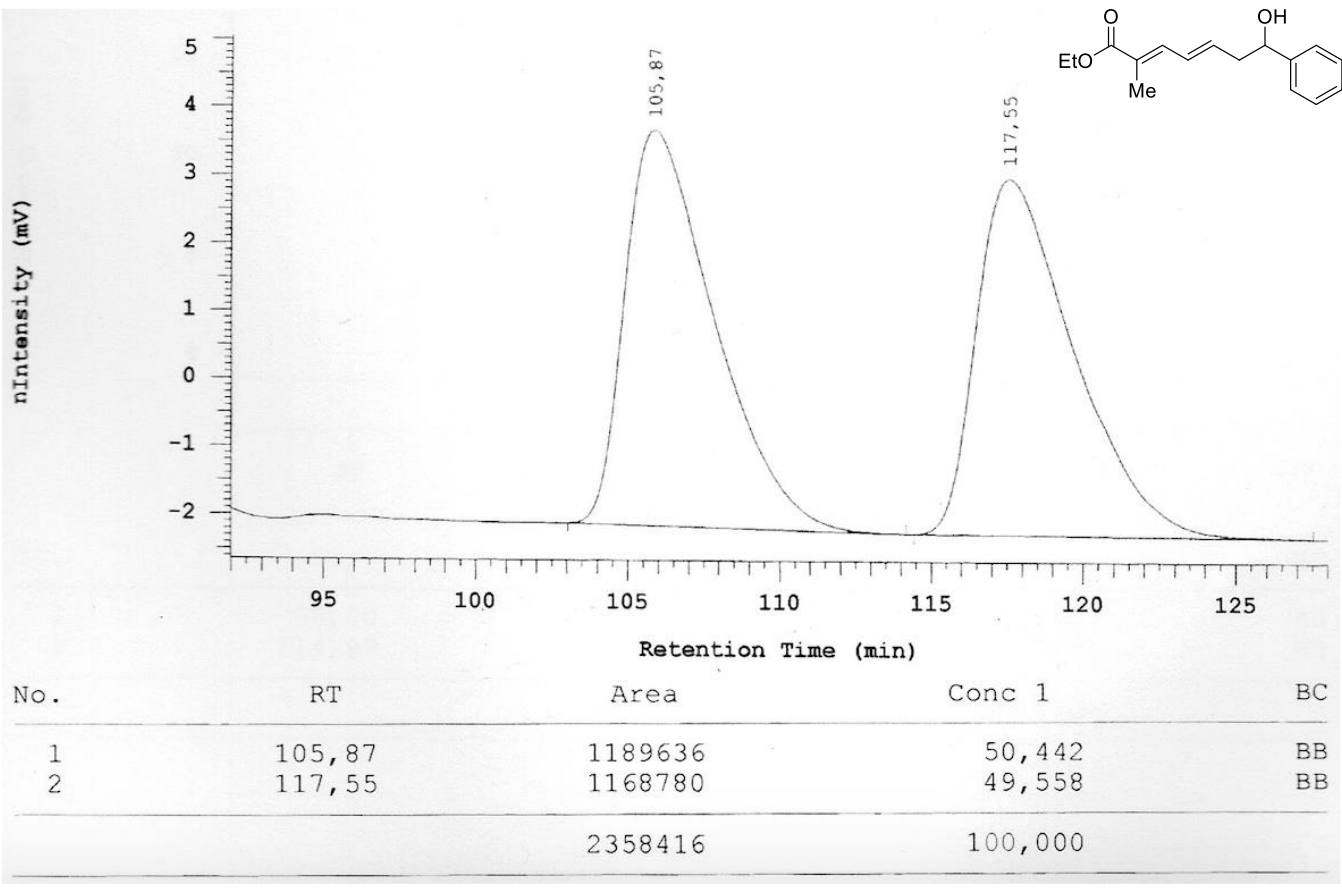

Chromatogram 1. Racemic sample of bVMAR product $\mathbf{5 a}$.

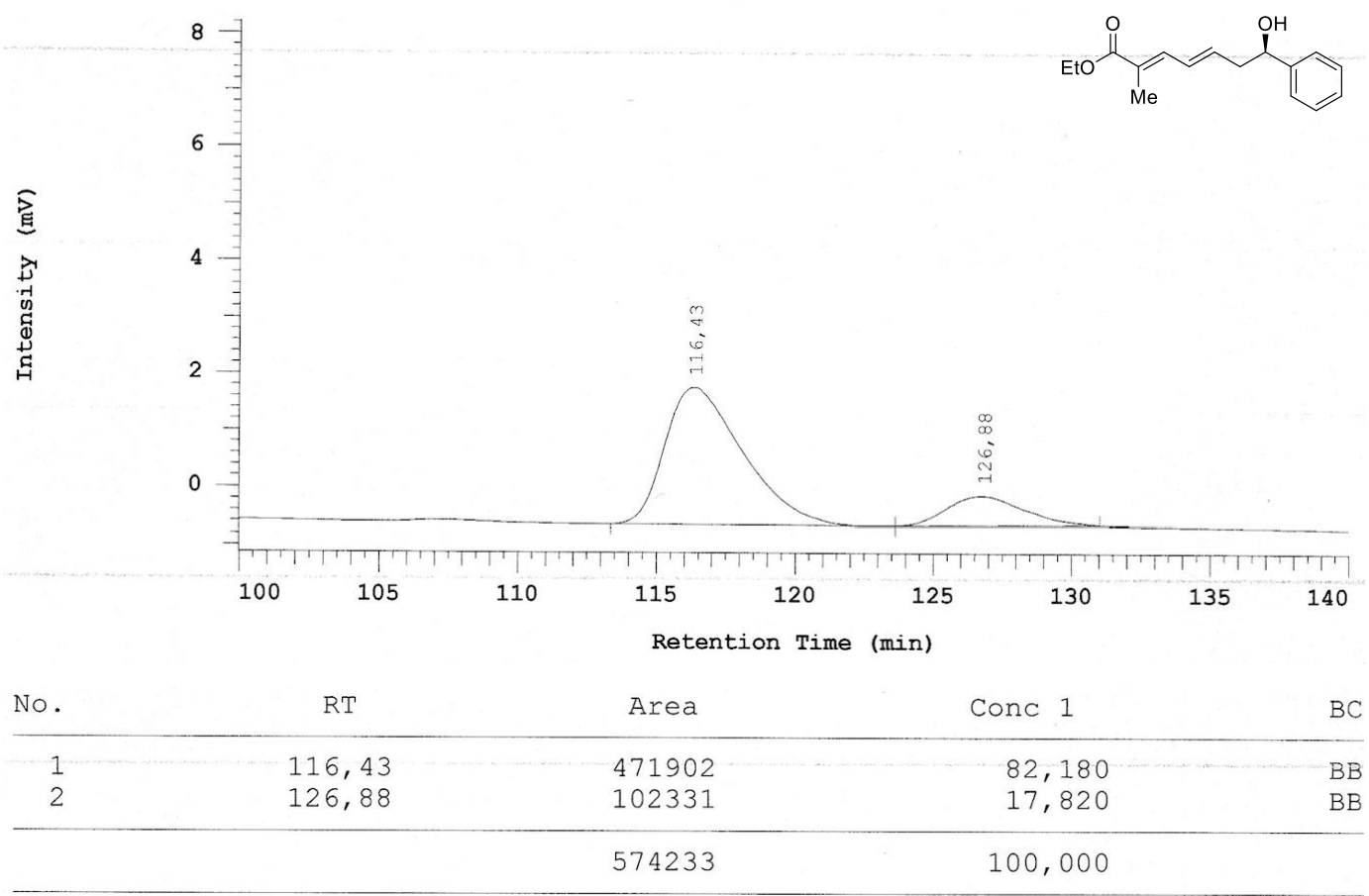

Chromatogram 2. Enantiomerically enriched sample of bVMAR product 5a. 


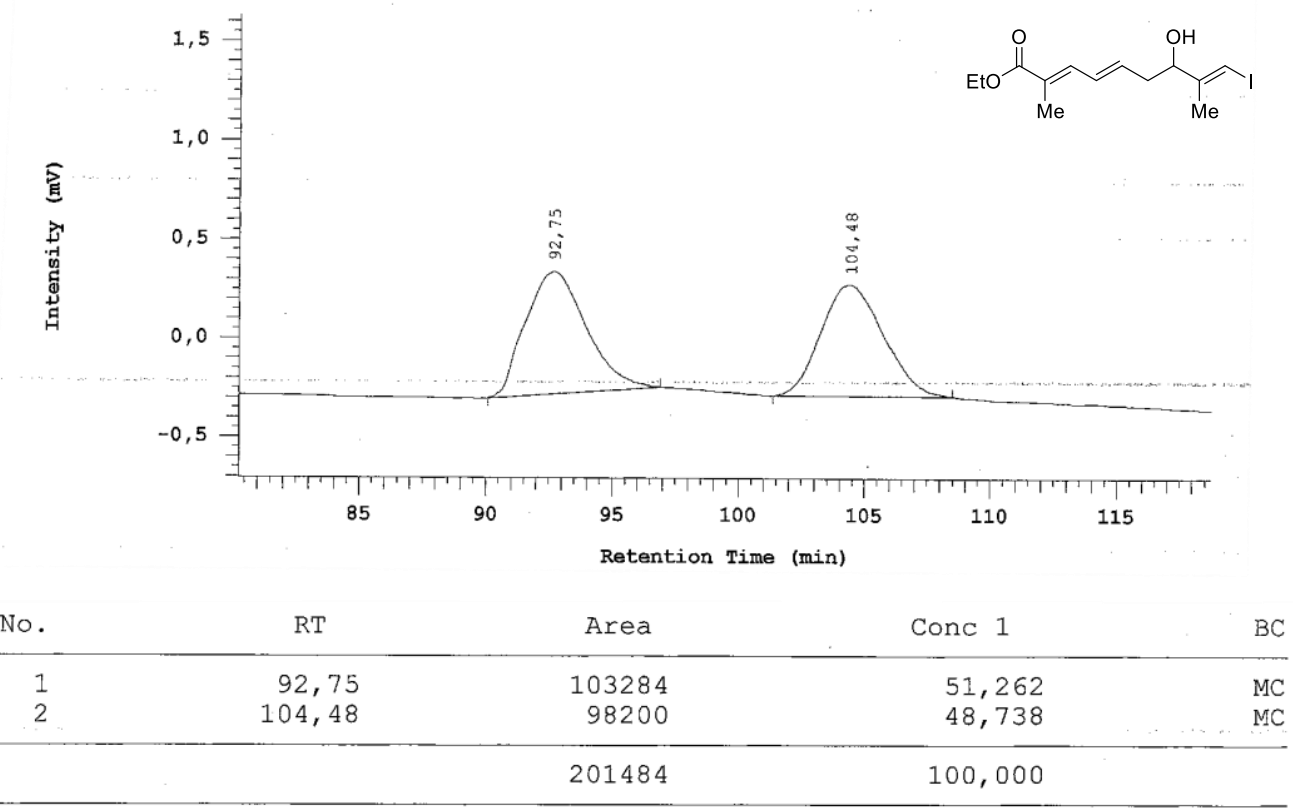

Chromatogram 3. Racemic sample of bVMAR product 5g.

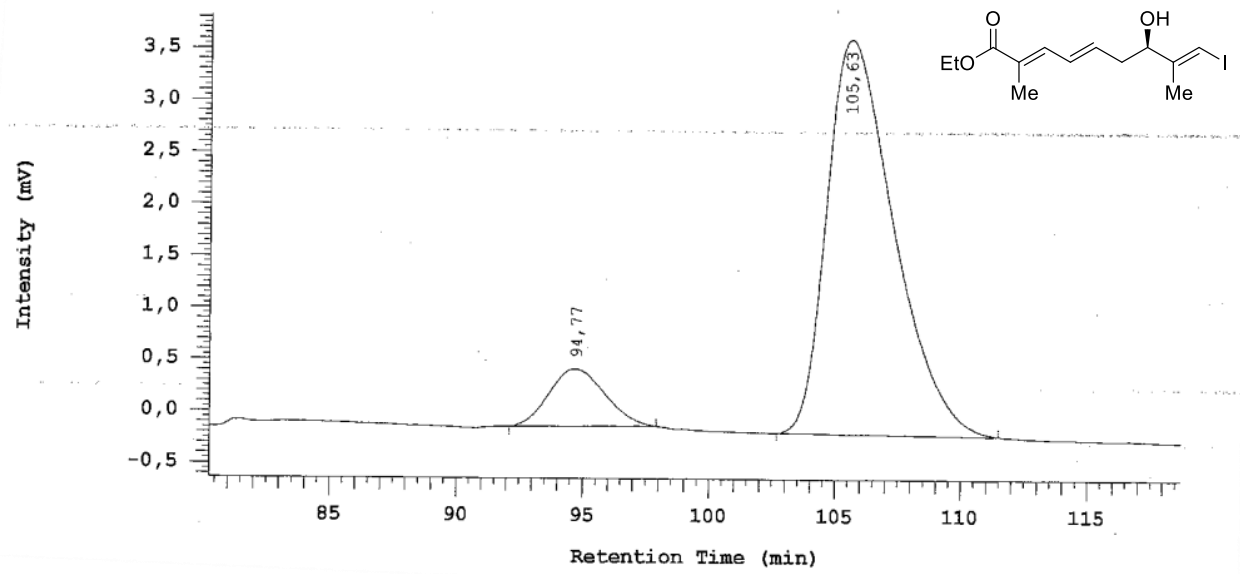

\begin{tabular}{|c|c|c|c|c|}
\hline No. & $\mathrm{RT}$ & Area & Conc 1 & $\cdots B C$ \\
\hline 1 & 94,77 & 84928 & 10,670 & MC \\
\hline 2 & 105,63 & 710995 & 89,330 & $\mathrm{BB}$ \\
\hline
\end{tabular}

Chromatogram 4. Enantiomerically enriched sample of bVMAR product $\mathbf{5 g}$. 
$e e$-determination - part 2: ${ }^{19} \mathrm{~F}$-NMR-data of the corresponding MTPAtes

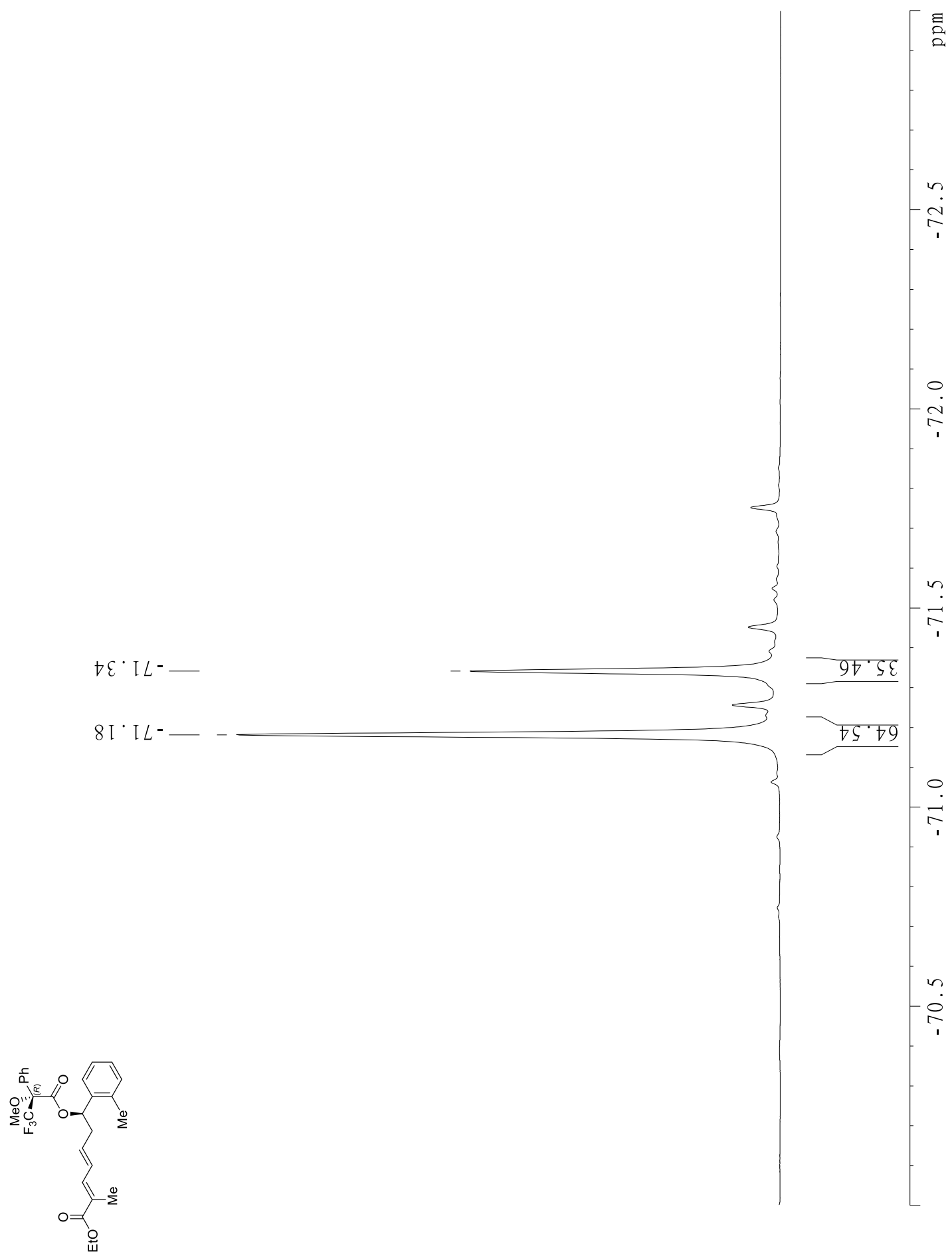

Spectrum $65 .{ }^{19} \mathrm{~F}$ spectrum of the $(R)$-Mosher ester of $\mathbf{5 b}$ measured in $\mathrm{CDCl}_{3}$ at $376 \mathrm{MHz}$. 


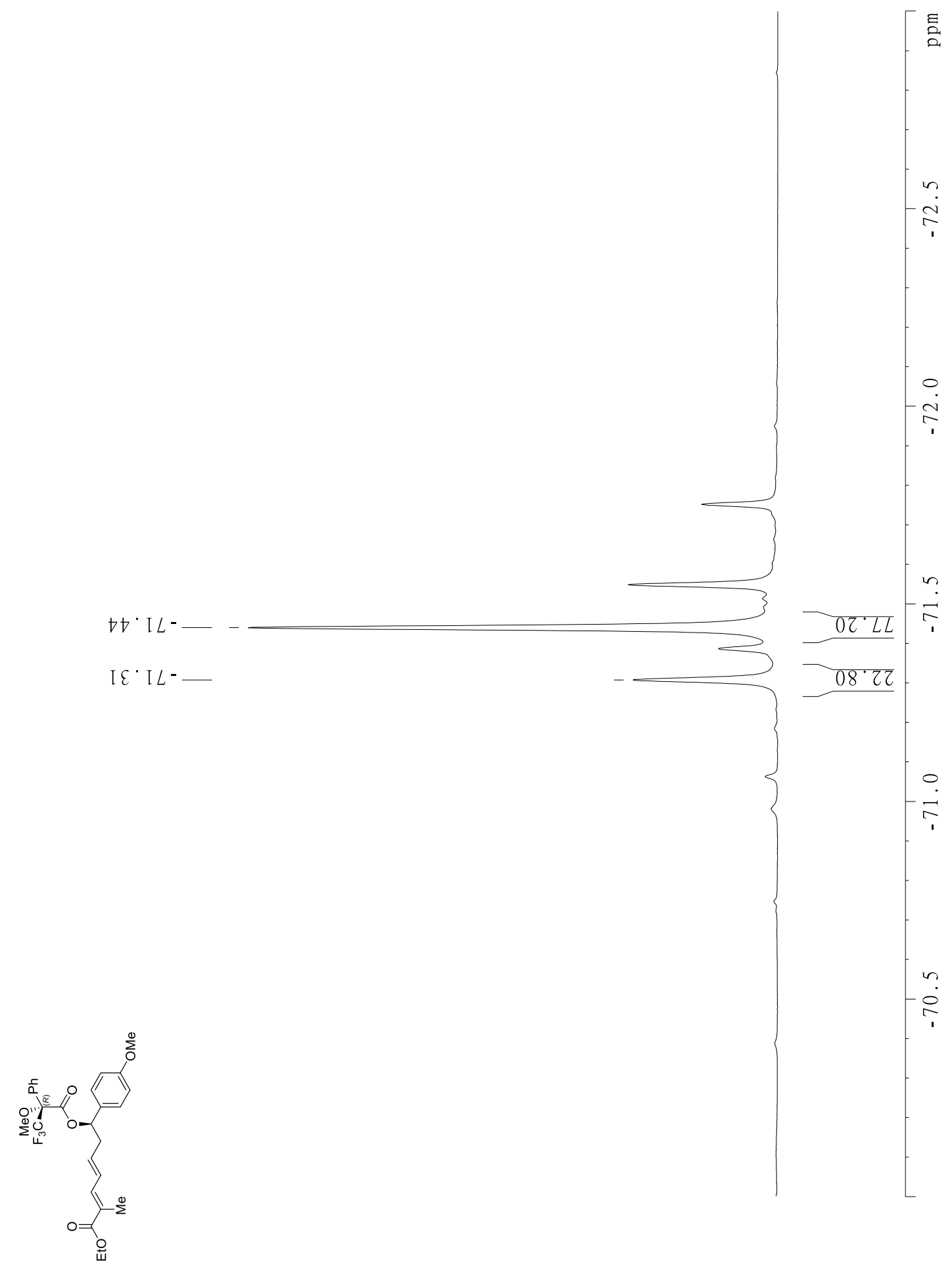

Spectrum 66. ${ }^{19} \mathrm{~F}$ spectrum of the $(R)$-Mosher ester of $\mathbf{5 c}$ measured in $\mathrm{CDCl}_{3}$ at $376 \mathrm{MHz}$. 


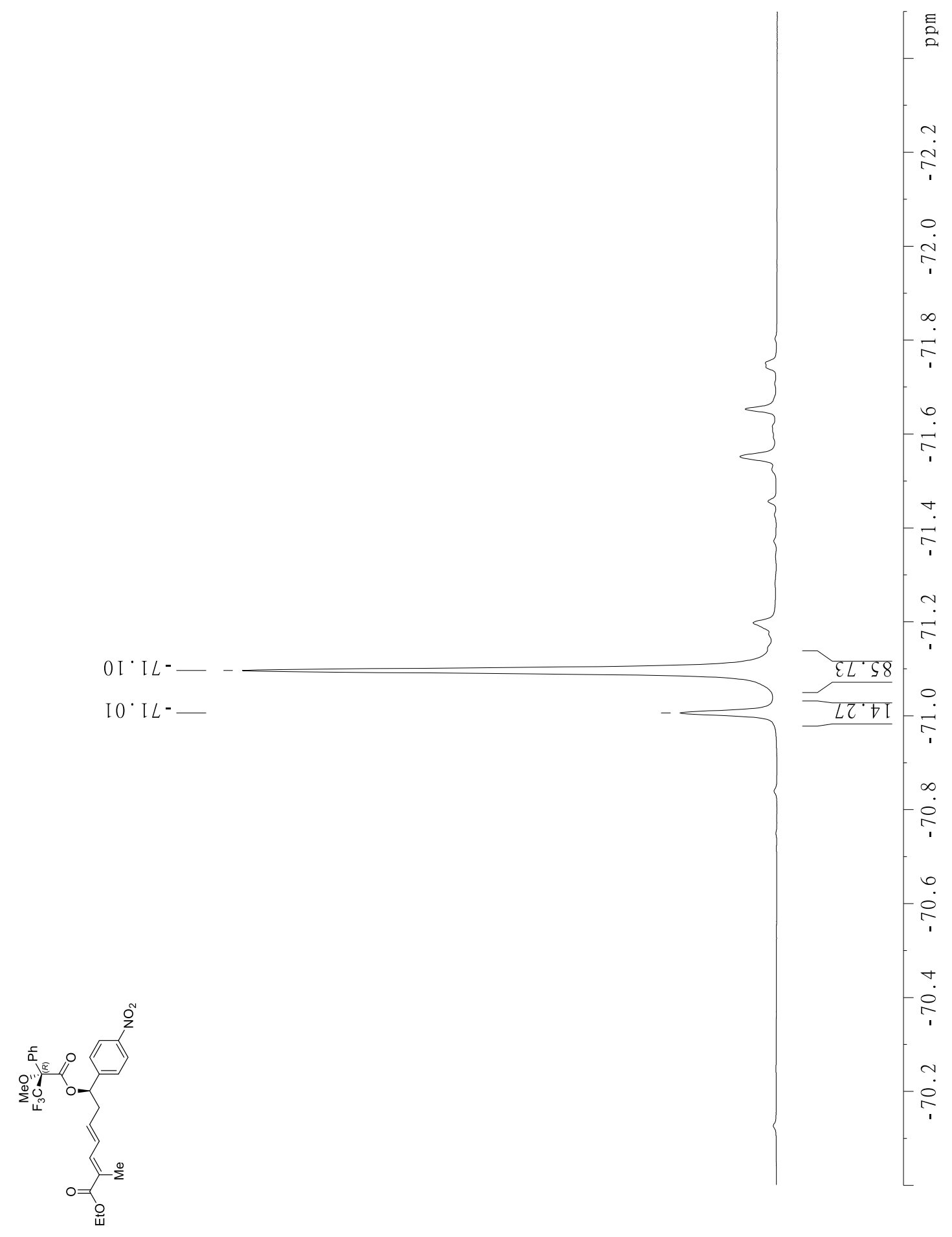

Spectrum 67. ${ }^{19} \mathrm{~F}$ spectrum of the $(R)$-Mosher ester of $\mathbf{5 d}$ measured in $\mathrm{CDCl}_{3}$ at $376 \mathrm{MHz}$. 


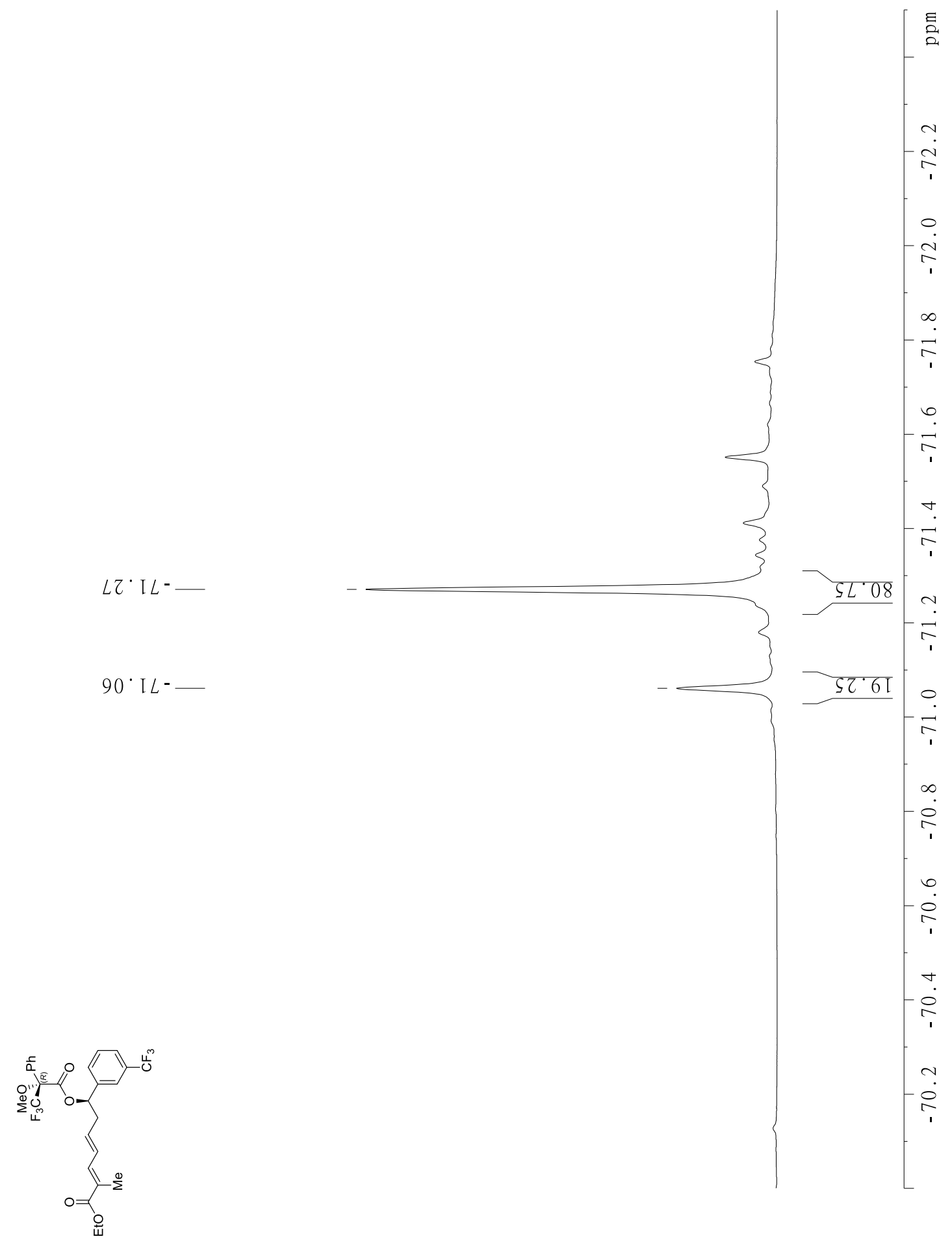

Spectrum 68. ${ }^{19} \mathrm{~F}$ spectrum of the $(R)$-Mosher ester of $\mathbf{5 e}$ measured in $\mathrm{CDCl}_{3}$ at $376 \mathrm{MHz}$. 


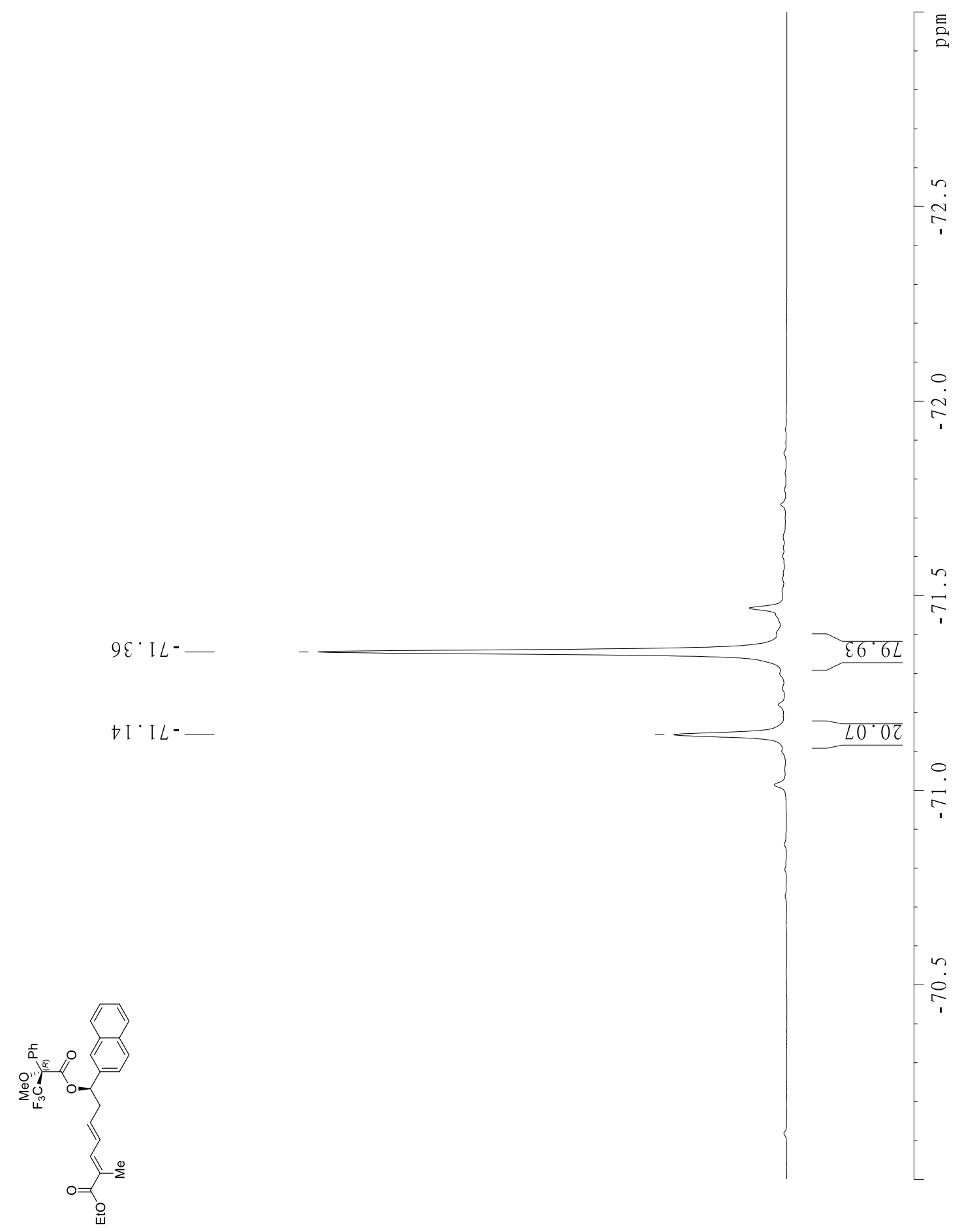

Spectrum 69. ${ }^{19} \mathrm{~F}$ spectrum of the $(R)$-Mosher ester of $\mathbf{5 f}$ measured in $\mathrm{CDCl}_{3}$ at $376 \mathrm{MHz}$. 


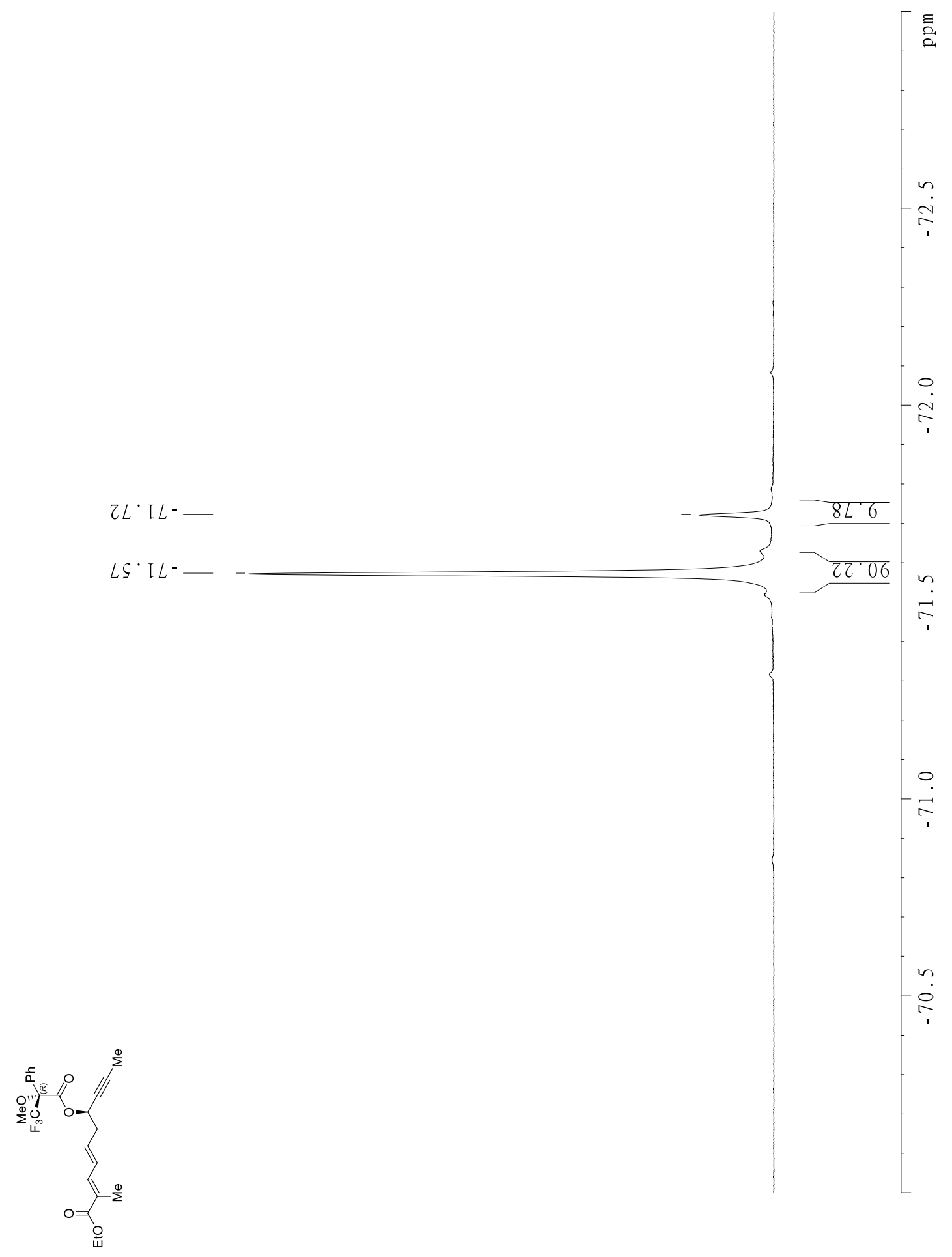

Spectrum 70. ${ }^{19} \mathrm{~F}$ spectrum of the $(R)$-Mosher ester of $\mathbf{5 h}$ measured in $\mathrm{CDCl}_{3}$ at $376 \mathrm{MHz}$. 


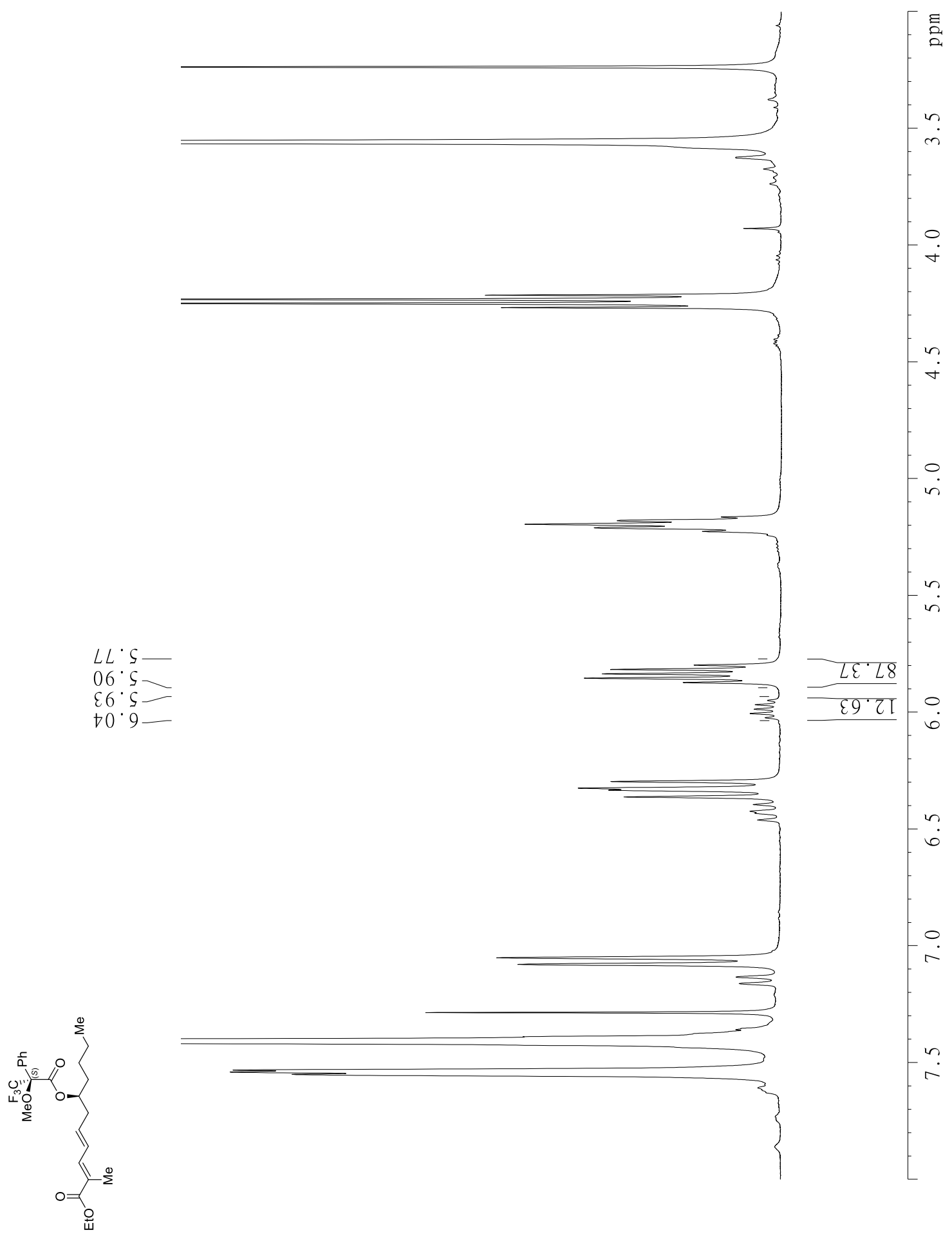

Spectrum 71. Enlarged view of the ${ }^{1} \mathrm{H}$ spectrum of the $(S)$-Mosher ester of $\mathbf{5 i}$ for the determination of the enantiomeric ratio; the spectrum was measured in $\mathrm{CDCl}_{3}$ at $400 \mathrm{MHz}$. 


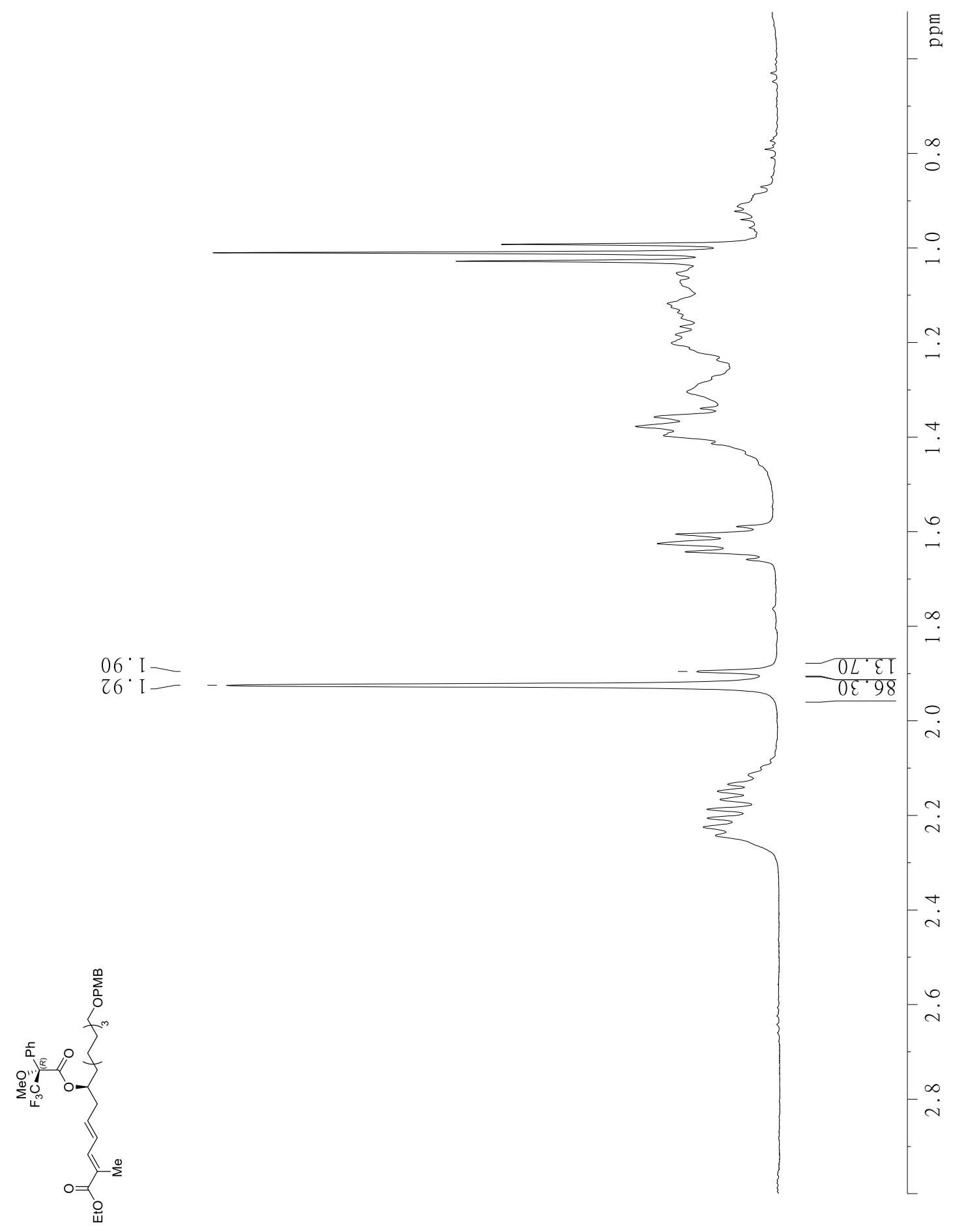

Spectrum 72. Enlarged view of the ${ }^{1} \mathrm{H}$ spectrum of the $(R)$-Mosher ester of $\mathbf{5} \mathbf{j}$ for the determination of the enantiomeric ratio; the spectrum was measured in $\mathrm{C}_{6} \mathrm{D}_{6}$ at $400 \mathrm{MHz}$. 


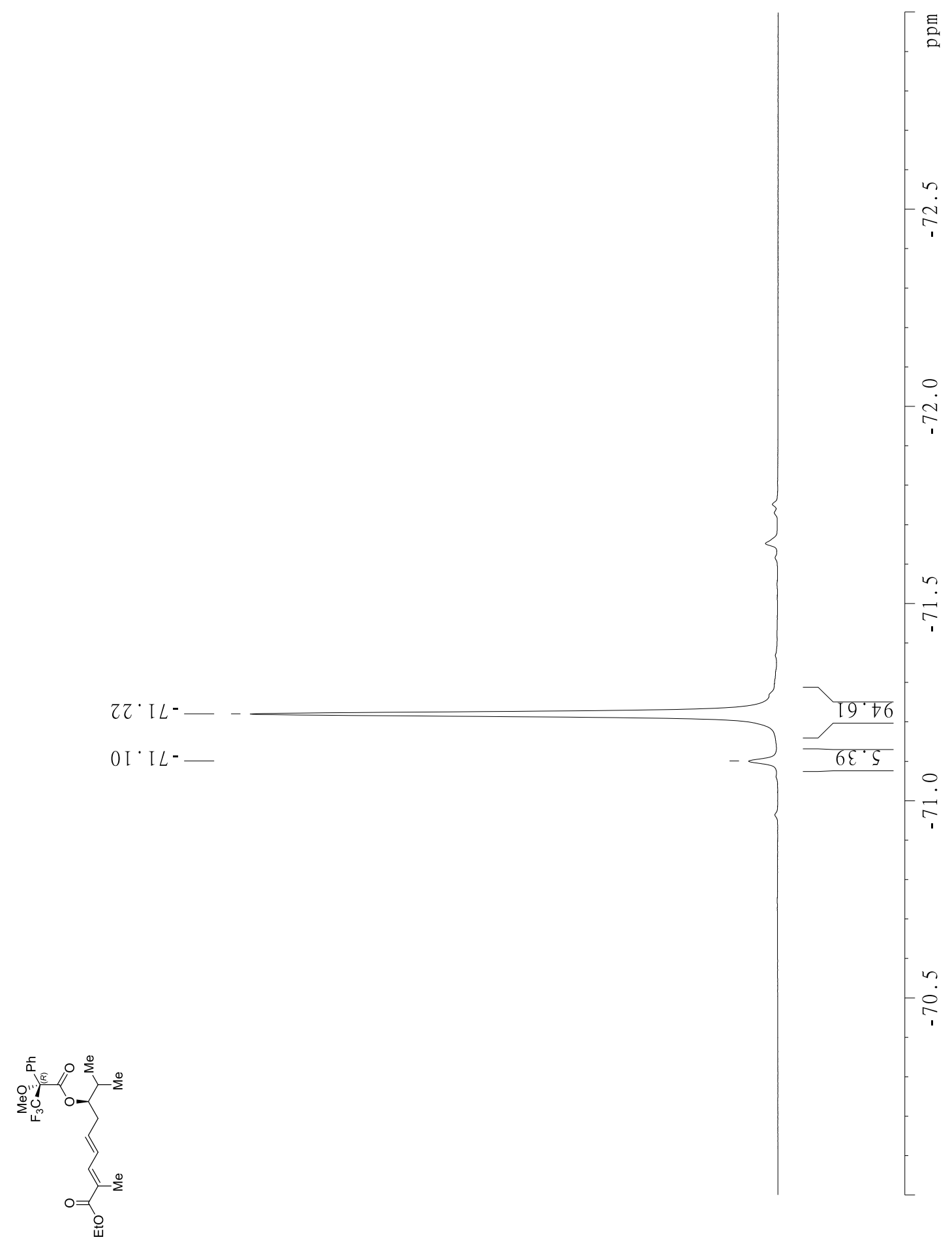

Spectrum 73. ${ }^{19} \mathrm{~F}$ spectrum of the $(R)$-Mosher ester of $\mathbf{5 k}$ measured in $\mathrm{CDCl}_{3}$ at $376 \mathrm{MHz}$. 


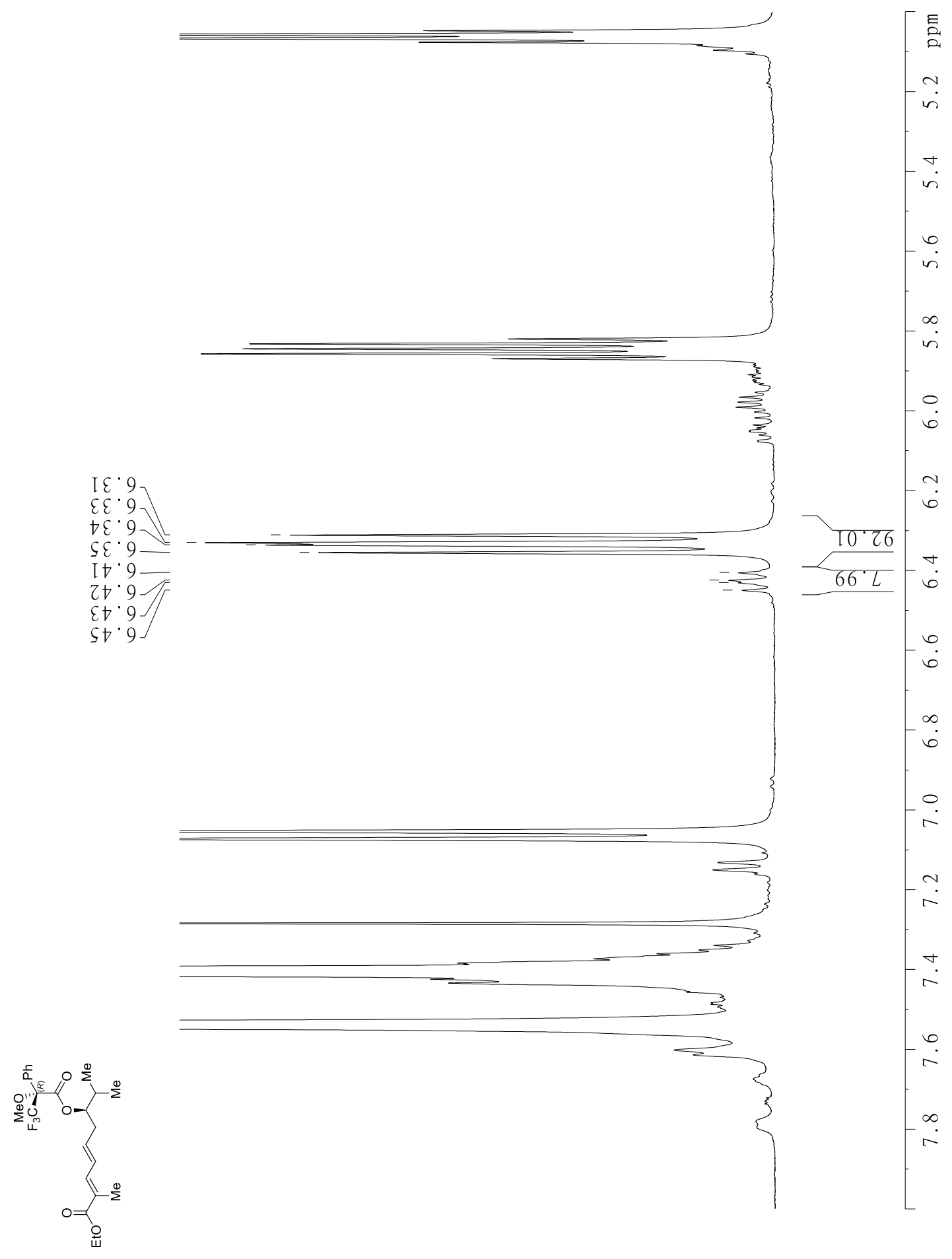

Spectrum 74. Enlarged view of the ${ }^{1} \mathrm{H}$ spectrum of the $(R)$-Mosher ester of $5 \mathbf{k}$ (obtained from the bVMAR reaction at a $1.2 \mathrm{mmol}$ scale) for the determination of the enantiomeric ratio; the spectrum was measured in $\mathrm{CDCl}_{3}$ at $600 \mathrm{MHz}$. 


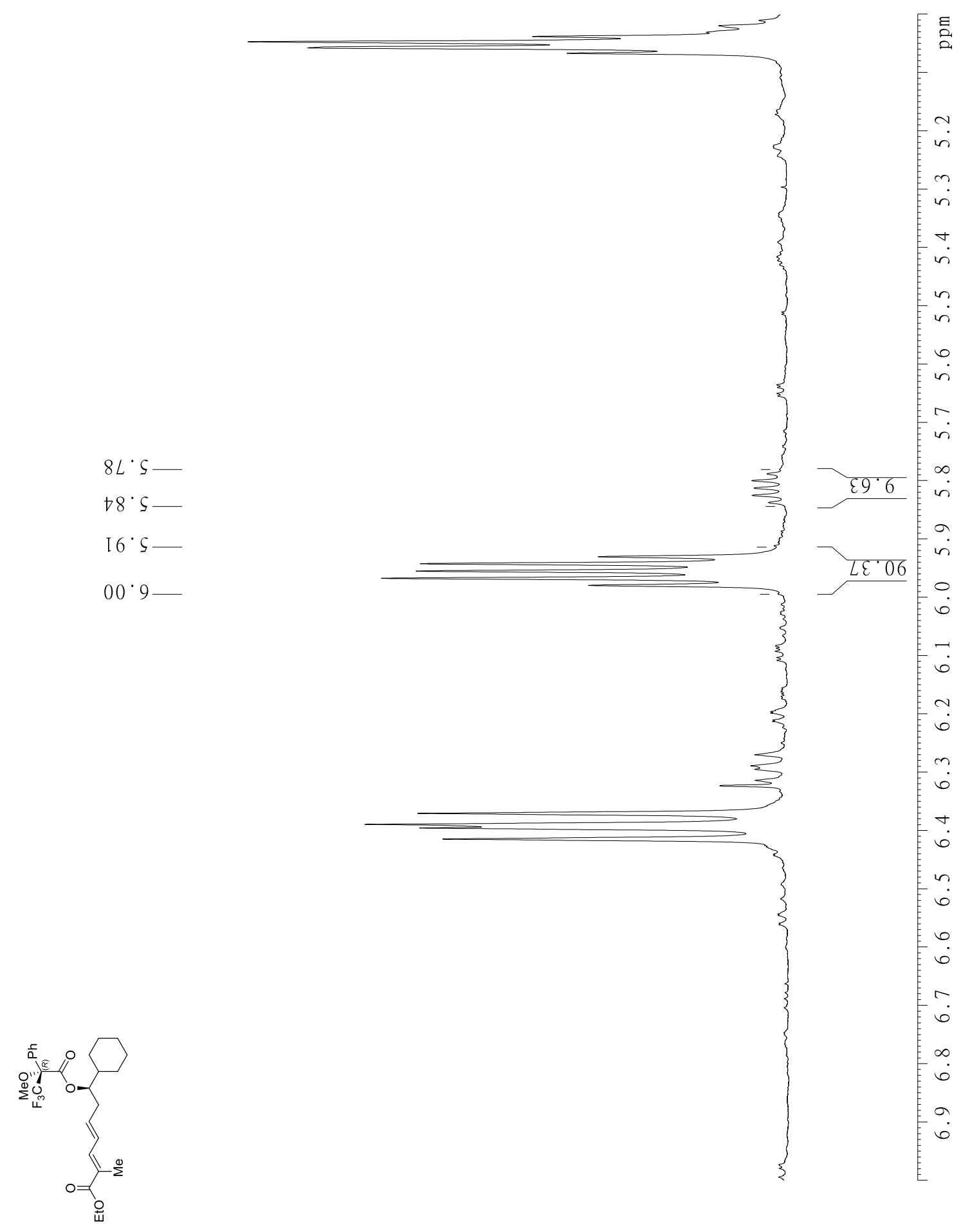

Spectrum 75. Enlarged view of the ${ }^{1} \mathrm{H}$ spectrum of the (R)-Mosher ester of $\mathbf{5 l}$ for the determination of the enantiomeric ratio; the spectrum was measured in $\mathrm{CDCl}_{3}$ at $600 \mathrm{MHz}$. 


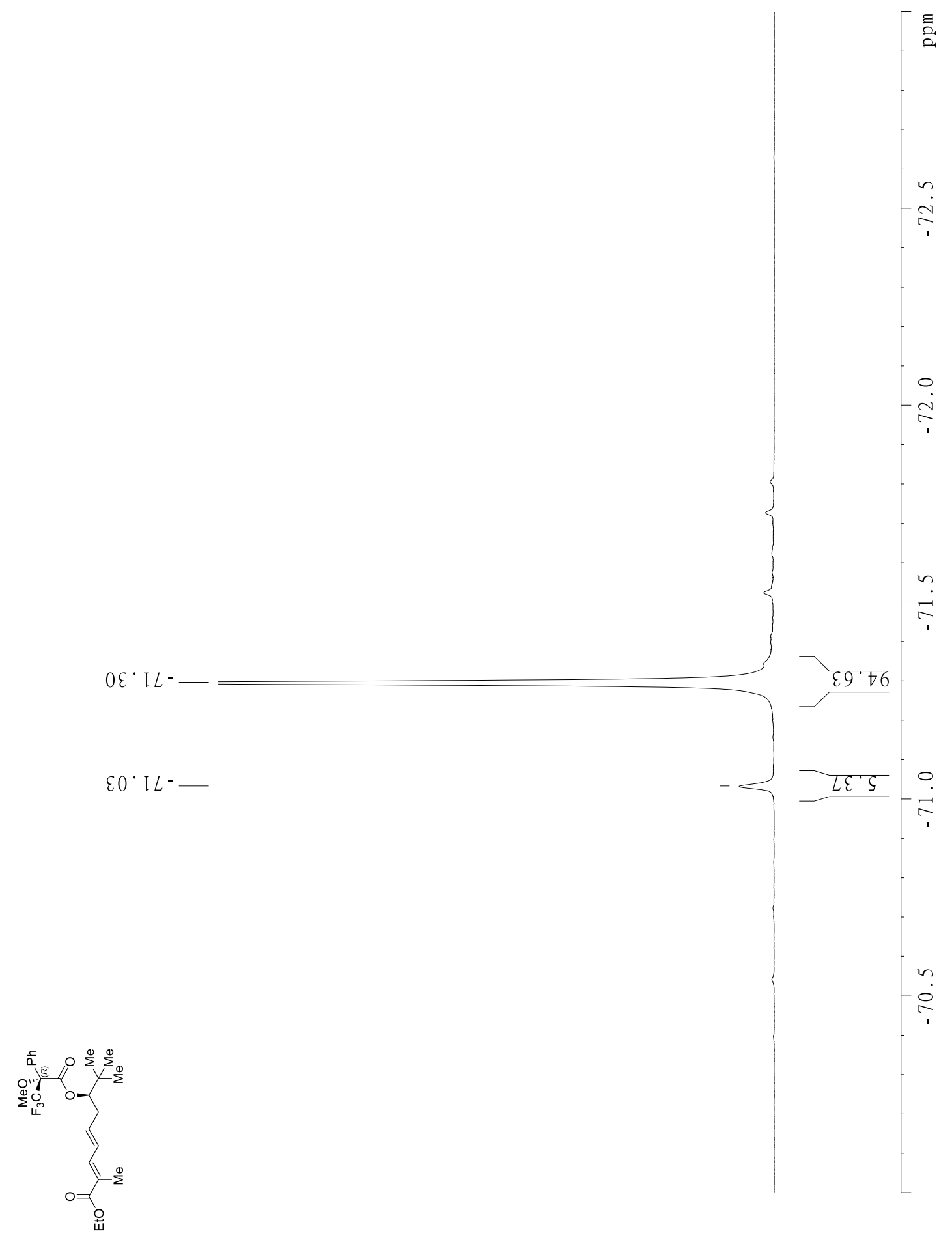

Spectrum 76. ${ }^{19} \mathrm{~F}$ spectrum of the $(R)$-Mosher ester of $\mathbf{5 m}$ measured in $\mathrm{CDCl}_{3}$ at $376 \mathrm{MHz}$. 


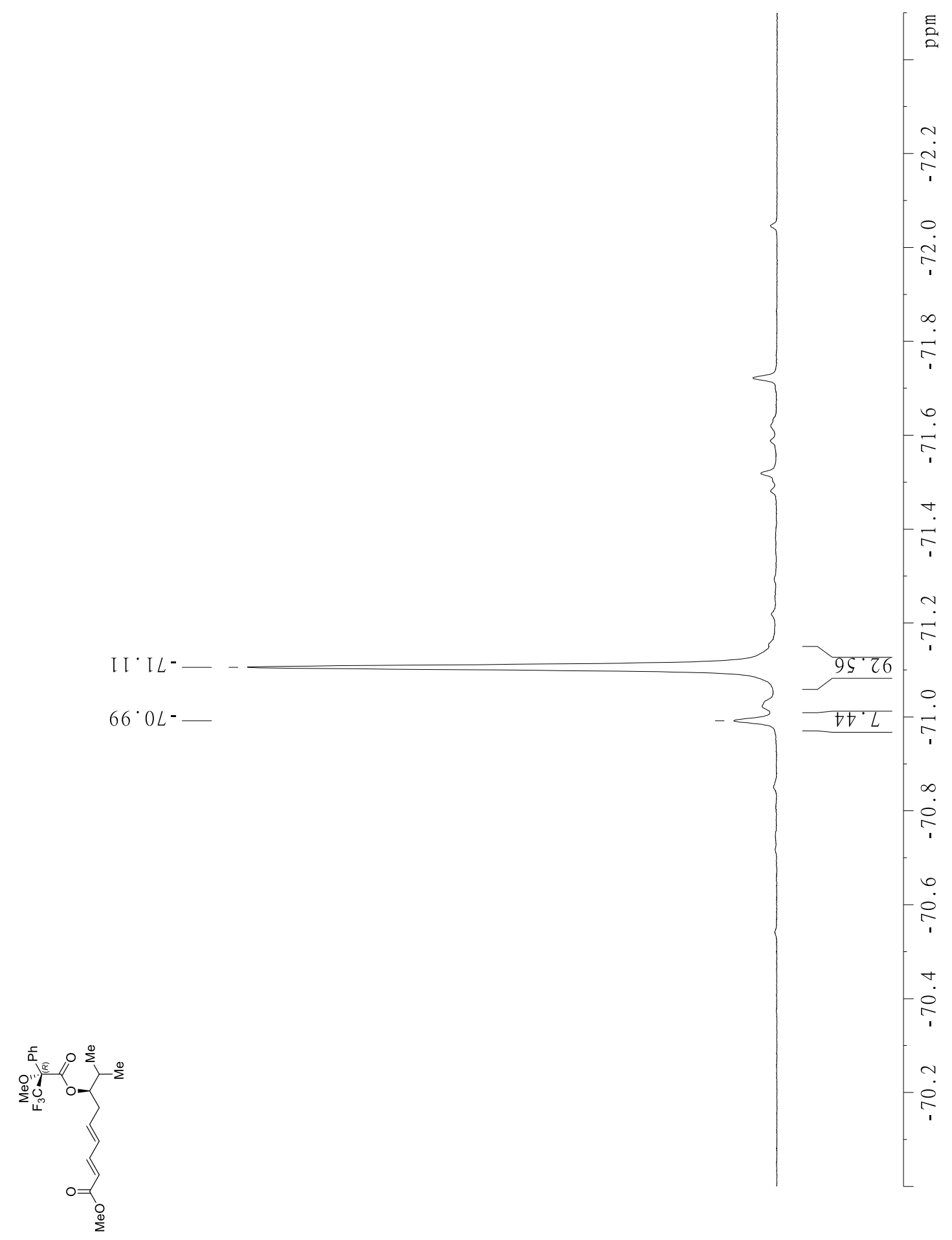

Spectrum 77. ${ }^{19} \mathrm{~F}$ spectrum of the $(R)$-Mosher ester of $\mathbf{5 n}$ measured in $\mathrm{CDCl}_{3}$ at $376 \mathrm{MHz}$. 


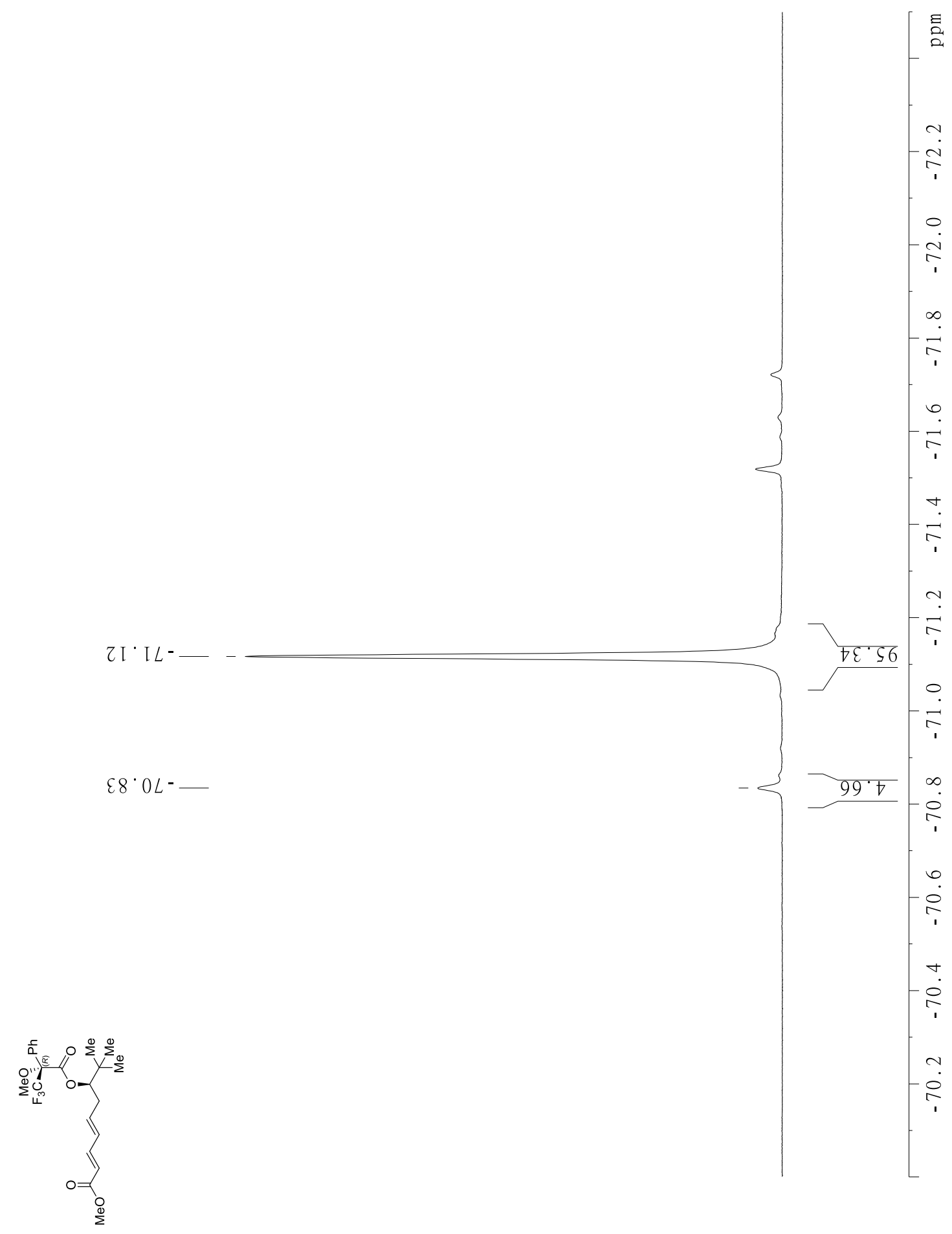

Spectrum 78. ${ }^{19} \mathrm{~F}$ spectrum of the $(R)$-Mosher ester of $\mathbf{5 o}$ measured in $\mathrm{CDCl}_{3}$ at $376 \mathrm{MHz}$. 